\title{
Who do we invent for? \\ Patents by women focus more on women's health, but few women get to invent
}

\author{
Rembrand Koning \\ Harvard Business School \\ Sampsa Samila \\ IESE \\ rem@hbs.edu \\ ssamila@iese.edu \\ John-Paul Ferguson \\ McGill University \\ john-paul.ferguson@mcgill.ca
}

15 March 2021

\begin{abstract}
Women engage in less commercial patenting and invention than men, which may affect what is invented. Using text analysis of all US biomedical patents filed from 1976 through 2010, we find that patents with all-female inventor teams are $35 \%$ more likely to focus on women's health than all-male teams. This effect holds over decades and across research areas. We also find that female researchers are more likely to discover female-focused ideas. These findings suggest that the inventor gender gap is partially responsible for thousands of missing female-focused inventions since 1976. More generally, our findings suggest that who benefits from innovation depends on who gets to invent.
\end{abstract}


The inventor gender gap is well established. While progress has been made, women are still less likely to enter STEM occupations, less likely to continue in scientific careers, and less likely to become inventors (1-6). Even today, only 35\% of STEM scientists and $13 \%$ of US patent inventors are women, suggesting that there are many "lost Curies," talented girls who never grow up to discover and invent (7-9).

While the gap itself is cause for concern, its consequences may extend beyond the labor market (10). It is possible that women are more likely to invent for women, and if so, the dearth of female inventors may cause society to supply too few inventions that benefit women. In fact, recent work suggests a shortage of projects, procedures, and products aimed at female patients' needs (11-13). Anecdotal evidence abounds that a disproportionate share of female-focused inventions - ovarian cancer tests, personalized breast cancer trials, cataract removal procedures ${ }^{1}$ - are invented and brought to market by women (14-16).

Yet there is little systematic evidence on whether female-invented products are more likely to focus on the needs of women, especially in knowledge-intensive domains like biomedicine. On the one hand, the lived experiences of female inventors may lead them to see opportunities to invent for people like themselves, specialize in clinical and disease areas that men may overlook, and invent with more knowledge of women's health $(17,18)$. On the other hand, medical inventors generally have extensive training in MD and $\mathrm{PhD}$ programs, which could eliminate knowledge differences between male and female researchers. Furthermore, inventions follow market demand (19). It could be that inventors, male or female, simply invent where the market pulls them.

Here, we measure the gender of inventors and the sex focus of their inventions for all U.S. biomedical patents filed between 1976 and 2010. We find that, in this field, inventions by women are more likely to focus on the medical needs of women. This pattern is strongest for all-female invention teams, holds over decades, and is present even within narrow areas of invention. This last finding suggests that the female inventor-invention link is both the result of women working in more female-focused research areas and female inventors identifying 
opportunities to invent for women regardless of the area in which they work. We also analyze biomedical research articles between 2002 and 2020, and we find that female-discovered ideas are also more likely to be female-focused. That upstream research ideas also exhibit a female inventor-invention link further suggests that the gender gap in who commercializes their ideas has contributed to the sex gap in what types of ideas become inventions.

The starting point for our analysis is a new measure for a patent's focus on the medical needs of men and/or women. We extract the title, abstract, and start of the summary text from the 441,504 "Drugs and Medical" patents in the PatentsView-NBER dataset. We then feed this text through the National Library of Medicine's Medical Text Indexer (MTI) (20). The MTI algorithm uses machine learning to map text to the Medical Subject Heading (MeSH) terms that most likely reflect the text's content. S1 gives more details.

The MeSH ontology includes two tags for the sex focus of the patent. A patent's text gets the "Female" tag when it covers "female organs, diseases, physiologic processes, genetics, etc." There is a parallel "Male" tag. We refer to the $12.76 \%$ of patents the MTI tagged "Female" as female-focused patents. Conversely, $13.27 \%$ of patents are male-focused. In S2 we validate the MTI algorithm, showing that it works well with patent text and that its accuracy is unbiased with respect to inventor gender. In S3 we show that, while the female and male MeSH tags are a coarse measure of sex focus, they capture significant differences in the likelihood that a patent benefits women or men. For example, with wordembedding methods and clinical-trial data we show that female- and male-tagged patents are roughly $400 \%$ more likely to describe inventions that would be evaluated by a female- or male-only trial. Furthermore, with data on disease incidence we show that patents tagged as female address diseases that impact roughly twice as many women; we find a similar pattern for male-tagged patents and men. We measure inventor gender using a standard dictionary-matching process (See S4 for details). Our final sample includes 430,060 patents with inventor-gender and sex-focus measures.

Our first set of analyses explores raw trends in the gender composition of inventors. We 
do so by splitting our data into two groups: patents with majority-female inventing teams ( $\geq 50 \%$ women) and patents with strictly more male inventors ( $>50 \%$ men). In Figure 1 we show that women-invented biomedical patents are on the rise, growing from $6.3 \%$ in 1976 to $16.2 \%$ in 2010 . Yet the 3,014 inventions by female-majority teams in 2010 are still outnumbered by the 3,347 patents invented by men nearly four decades earlier, in 1976 . In total, male-majority teams generated 373,774 patents, female-majority teams 56,286.

[Figure 1 about here.]

Figure 2 shows trends in the sex focus of invention for male-majority teams (A) and female-majority teams (B), with the black solid line showing the percentage of patents that are female-focused and the gray dashed line male-focused. Panel 2A shows that malemajority teams invented more for men than for women in all but one year of our data, though the difference has shrunk. In 1976, patents by male-majority teams were $15 \%$ more male-focused $(11.2 \%)$ than female-focused (9.7\%); in 2010, 6.2\% more male-focused $(13.7 \%)$ than female-focused (12.9\%). Intriguingly, this narrowing follows efforts by the NIH and NSF starting in the late 1980s to promote research on women's health (21). Overall, of the 373,774 patents invented by male-majority teams, 49,886 (13.3\%) were male-focused, 46,453 (12.4\%) were female-focused, while the rest were tagged as not sex-focused.

[Figure 2 about here.]

Panel 2B shows that female-majority teams are substantially more likely to invent for women and only slightly less likely to invent for men than male-majority teams are. In fact, in 1976, patents by female-majority teams were $7 \%$ more male-focused (12\% vs. 11.2\%) and $37 \%$ more female-focused (13.3\% vs. 9.7\%) than patents by men. As with male-majority teams, by 2010, female-majority teams invented more often for both sexes. On average, of the 56,286 patents invented by female-majority teams, 8,246 (14.7\%) were female-focused, $7,053(12.5 \%)$ were male-focused, and the rest were not tagged as sex-focused. Compared to 
male-majority teams, female-majority teams are only $6 \%(12.5 \% / 13.3 \%)$ less likely to invent for men, but they are $18.5 \%(14.7 \% / 12.4 \%)$ more likely to invent for women.

Figure 3 shows that the inventor gender gap amplifies these invention sex gaps. Panel 3A shows the number of female-focused minus male-focused patents invented by male-majority teams over time. Such teams regularly invent over one hundred additional male-focused inventions per year. Panel 3B shows the same difference for female-majority teams. Male and female inventors seem to have offsetting tendencies; but because there have been so many more male inventors, in aggregate invention has been skewed toward men's health (Panel C). The rising share of female inventors, and their increased focus on the needs of women, has begun to close and in some years flip the invention sex gap.

[Figure 3 about here.]

To further explore the magnitude and consequences of this female inventor-invention link we turn to patent-level models that regress a patent's female focus on whether the invention team falls into one of four buckets: all-female, majority (50\% or more) but not all female, minority-female, and all-male (our excluded baseline). Our model includes fixed effects for the year interacted with the patent's subcategory (Drugs, Surgery \& Instruments, Biotechnology, or Other) and the patent team's size (22). See S5 for additional details and tables. The estimates from this model, shown in black in Figure 4A, show a clear dose-response relationship increasing from $1.3 \pm 0.16( \pm S . E).(p<0.001)$ percentage points for minority-female to $2.4 \pm 0.19(p<0.001)$ for majority-female teams, and to $4.6 \pm 0.30$ $(p<0.001)$ for all-female teams. These reflect relative increases of 10\%, 18\%, and 35\% over the baseline female-focused invention rate of all-male teams.

[Figure 4 about here.]

These estimates are the result of two possible pathways. First, women might work in more female-focused research areas. Second, women might draw on different experiences and 
knowledge, regardless of their research area. If the first pathway is the main one, then the benefit to women of there being more female inventors will be concentrated in areas that are already female-focused (E.g., additional pre-eclampsia treatments). If the second pathway matters more, then women would also see benefits in research areas without a strong prior sex focus (E.g., female-focused atrial fibrillation treatments).

To isolate the within-research-area mechanism we match female-invented patents to maleinvented patents using the patent's publication year, subcategory, team size, and the narrow MeSH level-four disease area most associated with the invention (E.g., 1983 drug patents for atrial flutter [C14.280.067.248] with two inventors, or 1990 surgery patents for atrial fibrillation [C14.280.067.198] with three inventors). Figure 4A's estimates in gray come from this matched sample, which is roughly a tenth the size of our full sample, and includes year $\times$ subcategory $\times$ team size $\times$ disease fixed effects. After tightly matching within research areas, just over half of the total effect remains, with effect sizes for minority, majority, and all-female teams of $0.67 \pm 0.37(p=0.36), 1.3 \pm 0.37(p=0.002)$, and $2.8 \pm 0.55(p<0.001)$ percentage points. In other words, the female inventor-invention link stems equally from women working in female-focused research areas and from women spotting opportunities in other research areas.

In the supplemental materials we report additional analyses. In $\mathrm{S} 6$ we show that inventions by women are not merely more sex-focused in general, which could be so if women work in applied areas where anatomical sex differences are more central or if women are more likely to run sex and gender analyses (23). Instead, and consistent with Figure 2, we find teams with female inventors, compared to all-male inventor teams, are neither more nor less likely to invent for men. S7 presents robustness checks, including analyses showing that patents with all-female teams target diseases that impact $16 \%$ more women and are roughly $40 \%$ more likely to describe an invention that would be evaluated by a clinical trial that only enrolls women. S8 shows that our within-disease-area findings hold if we instead rely on different matching criteria, multi-dimensional disease fixed effects, or lasso-style machine- 
learning methods, all of which further account for differences in where women and men invent.

In S9 we explore heterogeneity in the female inventor-invention link. We find little evidence that the female inventor-invention link has consistently increased or decreased with time. We find suggestive evidence that all-female teams are 2.4 percentage points more likely to invent for women in non-corporate than in corporate settings, which is consistent with the idea that limited women's representation in corporate management leads firms to overlook female-focused R\&D opportunities (24). We also show that all-female teams are 8.7 percentage points more likely to invent for women in the surgery subcategory, versus 2.3 percentage points in drugs. This last difference is consistent with the idea that the historical hostility towards women in surgery (25) has both involved men dismissing promising female-focused surgical ideas and women being especially likely to develop those ideas.

Overall, we find that women's biomedical inventions are more likely to focus on women's needs than men's inventions are. That said, patents are a downstream outcome in the process of discovery and invention. Perhaps nascent female inventors discover ideas for both sexes equally, but gender stereotypes lead women to not patent their male-focused ideas (or men to not patent their female-focused ones). Differential selection into patenting, not differences in the knowledge and experiences that women bring to the discovery process, might be responsible.

To test for differential selection, we use data on the upstream research ideas that biomedical patents build on. Specifically, we analyze 2,062,695 original biomedical research articles from the National Library of Medicine's PubMed database that are at risk of being commercialized and were published between 2002 (the first year we have data on the authors' gender) and 2020 (S10 provides further details). We again use the MeSH ontology's "Male" and "Female" check tags to asses articles' sex focus, but we do not use the MTI algorithm, because articles in PubMed are assigned MeSH tags by human medical indexers.

Panel 4B mirrors Panel 4A, but shows that research teams with more women are more 
likely to discover for women. There is again a clear dose-response relationship, with the effect increasing from $0.84 \pm 0.08(p<0.001)$ to $3 \pm 0.08(p<0.001)$ to $4.5 \pm 0.12(p<0.001)$ percentage points for minority, majority, and all-female teams. As in $4 \mathrm{~A}$, the gray estimates in $4 \mathrm{~B}$ involve matching and fixed effects to account for differences in men's and women's publishing rates in different narrow disease areas (S11). While the coefficients shrink towards zero, the differences are largely non-significant. Compared to the baseline female-focused publication rate, papers with all-female teams are about $12 \%$ more likely to focus on the health needs of women. S12 reports additional robustness tests, including analyses that show that discoveries by women are not more likely to be male-focused, that our findings hold under alternative matching schemes, and that discoveries by all-female teams target diseases that impact $47 \%$ more women and are over $40 \%$ more likely to describe an idea that would be evaluated by a clinical trial that only enrolls women.

These findings imply that many promising female-focused discoveries have yet to be commercialized, because women are less likely to obtain patents $(1,26)$. Back-of-the-envelope calculations (S13) suggest that if all the patents invented between 1976 and 2010 had been produced by men and women equally then there would have been roughly 6,500 more femalefocused inventions. Even if we focus within research areas, thus holding constant the number of patents produced across areas, we still find that inventor gender equality would have resulted in just over 3,500 more female-focused inventions. If research articles were produced equally by men and women then from 2002 to 2020 there would have potentially been 40,000 more female-focused discoveries. This suggest that there may still be many untapped market opportunities to invent for women, opportunities that could in turn improve women's health.

It is important to note that these estimates of the number of lost female inventions are suggestive. The primary goal of this paper is to establish a link between the background of inventors and who might benefit from their inventions. We do not observe the counterfactual world where there are more (or fewer) female inventors or scientists. Hence, the evidence presented here cannot tell us whether women's female-focused inventions could have instead 
been invented by all-male teams, or the extent to which female inventors might displace men from working on women's health, or whether women might instead pursue other, non-femalefocused ideas if there were a greater share of female inventors and inventions. Our analyses also cannot tell us if the gender mix of inventions is or ever has been socially optimal. Finally, while recent work finds that demographic similarity between doctors and patients improves female and African American health outcomes (18, 27, 28), our analysis does not shed light on whether female-invented and -focused patents are more original or impactful. We see each of these limitations of the present study as a promising avenue for more research.

That research should not be limited to the differences between men and women, nor to biomedicine. Our findings sketch a road map for future research on demographics and innovation. Reducing the barriers that disadvantaged groups face when working and inventing has accounted for a non-trivial share of economic growth over the last century (29). In virtually all cases, though, whether the population under study has been 19th-century Black inventors (30), early-20th-century Eastern European scientists (31), or some other group, the focus has been on how discrimination has reduced the overall level of innovation. By contrast, we find a robust and sizable connection between inventor gender and the sex focus of inventions. This is an important step in showing how labor-market bias might spill over into product-market bias. While earlier work on gender and innovation has focused on estimating biases against entrepreneurs and inventors while holding the underlying idea constant (e.g. (32)), we hope future work will focus on understanding bias against ideas that especially benefit historically disadvantaged groups (33). Our findings here suggest that inequities in who invents may lead to inequities in who benefits from invention. 


\section{Notes}

${ }^{1}$ Cataracts are more common in women than in men (34).

\section{References}

[1] Waverly W. Ding, Fiona Murray, and Toby E. Stuart. Gender differences in patenting in the academic life sciences. Science, 313:665-667, August 2006.

[2] J. Scott Long and Mary Frank Fox. Scientific careers: Universalism and particularism. Annual Review of Sociology, 21:45-71, 1995.

[3] Pierre Azoulay, Ryan Michigan, and Bhaven N. Sampat. The anatomy of medical school patenting. New England Journal of Medicine, (357):2049-2056, 2007.

[4] Fiona Murray and Leigh Graham. Buying science and selling science: Gender differences in the market for commercial science. Industrial and Corporate Change, 16(4):657-689, 2007.

[5] Marc J. Lerchenmueller and Olav Sorenson. The gender gap in early career transitions in the life sciences. Research Policy, 47:1007-1017, 2018.

[6] Shulamit Kahn and Donna Ginther. Women and STEM. Technical Report 23525, National Bureau of Economic Research, June 2017.

[7] Junming Huang, Alexander J Gates, Roberta Sinatra, and Albert-László Barabási. Historical comparison of gender inequality in scientific careers across countries and disciplines. Proceedings of the National Academy of Sciences, 117(9):4609-4616, 2020.

[8] Alex Bell, Raj Chetty, Xavier Jaravel, Neviana Petkova, and John Van Reenen. Who becomes an inventor in America? The importance of exposure to innovation. Quarterly Journal of Economics, 134 (2):647-713, 2019.

[9] Jessica Milli, Emma Williams-Baron, Meika Berlan, Jenny Xia, and Barbara Gault. Equity in innovation: Women inventors and patents. Institute for Women's Policy Research Report, 2016.

[10] Lisa S. Rotenstein and Anupam B. Jena. Lost Taussigs - The consequences of discrimination in medicine. New England Journal of Medicine, (378):2255-2257, 2018.

[11] Maya Dusenbery. Doing Harm: The Truth about How Bad Medicine and Lazy Science Leave Women Dismissed, Misdiagnosed, and Sick. HarperOne, New York, 2018.

[12] Caroline Criado Perez. Invisible Women: Data Bias in a World Designed for Men. Abrams Press, New York, 2019.

[13] Office of Research on Women's Health. Report of the advisory committee on research on women's health. Technical report, National Institute of Health, 2016. NIH Publication No. 17 OD 7995.

[14] Matthew Herper. A 32-year-old's startup, inspired by a health scare, sells for $\$ 275$ million. Forbes, April 2018.

[15] Katie Hafner. A breast cancer surgeon who keeps challenging the status quo. New York Times, September 2015.

[16] National Institute of Health. Changing the face of medicine: Dr. Patricia E. Bath. Technical report, National Institute of Health, 2019. https://cfmedicine.nlm.nih.gov/physicians/biography_26.html. 
[17] Londa Schiebinger, editor. Gendered Innovations in Science and Engineering. Stanford University Press, Palo Alto, CA, 2008.

[18] Brad Greenwood, Seth Carnahan, and Laura Huang. Patient-physician gender concordance and increased mortality among female heart attack patients. Proceedings of the National Academy of Sciences of the United States of America, 115(34), August 2018.

[19] Daron Acemoglu and Joshua Linn. Market size in innovation: Theory and evidence from the pharmaceutical industry. Quarterly Journal of Economics, 119(3):1049-1090, 2004.

[20] J. Mork, A. Aronson, and D. Demner-Fushman. 12 years on - Is the NLM medical text indexer still useful and relevant? Journal of Biomedical Semantics, 8(1):8, 2017.

[21] Nancy C. Lee. Progress in women's health: 1985-2015. Public Health Reports, 130(2):121-122, 2015.

[22] Stefan Wuchty, Benjamin F. Jones, and Brian Uzzi. The increasing dominance of teams in the production of knowledge. Science, 316(5827):1036-1039, 2007.

[23] Mathias Wullum Nielsen, Jens Peter Andersen, Londa Schiebinger, and Jesper W. Schneider. One and a half million medical papers reveal a link between author gender and attention to gender and sex analysis. Nature Human Behavior, 1:791-796, 2017.

[24] Kjersten Bunker Whittington and Laurel Smith-Doerr. Women inventors in context: Disparities in patenting across academia and industry. Gender \& Society, 22(2):194-218, 2008.

[25] Frances K Conley. Walking Out on the Boys. Macmillan, New York, 1999.

[26] Kyle Jensen, Balázs Kovács, and Olav Sorenson. Gender differences in obtaining and maintaining patent rights. Nature Biotechnology, 36(4):307-309, 2018.

[27] Marcella Alsan, Owen Garrick, and Grant Graziani. Does diversity matter for health? Experimental evidence from Oakland. American Economic Review, 109(12):4071-4111, 2019.

[28] Brad N Greenwood, Rachel R Hardeman, Laura Huang, and Aaron Sojourner. Physician-patient racial concordance and disparities in birthing mortality for newborns. Proceedings of the National Academy of Sciences, 117(35):21194-21200, 2020.

[29] Chang-Tai Hsieh, Erik Hurst, Charles I Jones, and Peter J Klenow. The allocation of talent and us economic growth. Econometrica, 87(5):1439-1474, 2019.

[30] Lisa D Cook. Violence and economic activity: Evidence from African American patents, 1870-1940. Journal of Economic Growth, 19(2):221-257, 2014.

[31] Petra Moser and Shmuel San. Immigration, science, and invention. evidence from the quota acts. Technical report, Working Paper, 2020.

[32] Alison Wood Brooks, Laura Huang, Sarah Wood Kearney, and Fiona E. Murray. Investors prefer entrepreneurial ventures pitched by attractive men. Proceedings of the National Academy of Sciences of the United States, 111(12):4427-4431, 2014.

[33] M. W. Nielsen, C. W. Bloch, and L. Schiebinger. Making gender diversity work for scientific discovery and innovation. Nature Human Behavior, 2018. https://doi.org/10.1038/s41562-018-0433-1.

[34] Lixia Lou, Xin Ye, and Peifang Xu. Association of sex with the global burden of cataract. JAMA Ophthalmology, 136(2):116-121, 2018. 
[35] Alan R. Aronson, James G. Mork, Clifford W. Gay, Susanne M. Humphrey, and Willie J. Rogers. The NLM indexing initiative's medical text indexer. Studies in Health Technology and Informatics, pages 268-272, 2004.

[36] Kevin J. Boudreau, Eva C. Guinan, Karim R. Lakhani, and Christoph Riedl. Looking across and looking beyond the knowledge frontier: Intellectual distance, novelty, and resource allocation in science. Management Science, 62(10):2765-2783, 2016.

[37] Alastair R Rae, James G Mork, and Dina Demner-Fushman. Convolutional neural network for automatic MeSH indexing. In Joint European Conference on Machine Learning and Knowledge Discovery in Databases, pages 581-594. Springer, 2019.

[38] Cassidy R Sugimoto, Yong-Yeol Ahn, Elise Smith, Benoit Macaluso, and Vincent Larivière. Factors affecting sex-related reporting in medical research: A cross-disciplinary bibliometric analysis. The Lancet, 393(10171):550-559, 2019.

[39] Burt L Monroe, Michael P Colaresi, and Kevin M Quinn. Fightin' words: Lexical feature selection and evaluation for identifying the content of political conflict. Political Analysis, 16(4):372-403, 2008.

[40] Christopher J. L. Murray and Alan D. Lopez. Measuring the global burden of disease. New England Journal of Medicine, 369(5):448-457, 2013.

[41] Piotr Bojanowski, Edouard Grave, Armand Joulin, and Tomas Mikolov. Enriching word vectors with subword information. arXiv preprint arXiv:160\%.04606, 2016.

[42] Armand Joulin, Edouard Grave, Piotr Bojanowski, and Tomas Mikolov. Bag of tricks for efficient text classification. arXiv preprint arXiv:1607.01759, 2016.

[43] Yijia Zhang, Qingyu Chen, Zhihao Yang, Hongfei Lin, and Zhiyong Lu. Biowordvec: Improving biomedical word embeddings with subword information and MeSH. Scientific Data, 6(1):1-9, 2019.

[44] Yanshan Wang, Sijia Liu, Naveed Afzal, Majid Rastegar-Mojarad, Liwei Wang, Feichen Shen, Paul Kingsbury, and Hongfang Liu. A comparison of word embeddings for biomedical natural language processing. Journal of Biomedical Informatics, 87:12-20, 2018.

[45] Daniel E. Ho, Kosuke Imai, Gary King, and Elizabeth A. Stuart. Matching as nonparametric preprocessing for reducing model dependence in parametric causal inference. Political Analysis, 15(3):199-236, 2007.

[46] Victor Chernozhukov, Christian Hansen, and Martin Spindler. Valid post-selection and postregularization inference: An elementary, general approach. Annual Reviews of Economics, 2015.

[47] James Evans. Industry induces academic science to know less about more. American Journal of Sociology, 116(2):389-452, 2010.

[48] Kyle Myers. The elasticity of science. American Economic Journal: Applied Economics, 12(4):103-34, 2020.

[49] Pierre Azoulay, Joshua S. Graff Zivin, Danielle Li, and Bhaven N. Sampat. Public R\&D investments and private-sector patenting: Evidence from NIH funding rules. Review of Economic Studies, 86:117-152, 2019.

[50] Michaël Bikard and Matt Marx. Bridging academia and industry: How geographic hubs connect university science and corporate technology. Management Science, 66(8):3425-3443, 2020.

[51] Jevin D. West, Jennifer Jacquet, Molly M. King, Shelley J. Correll, and Carl T. Bergstrom. The role of gender in scholarly authorship. PLOS One, 2013. https://doi.org/10.1371/journal.pone.0066212. 
[52] Matt Marx and Aaron Fuegi. Reliance on science: Worldwide front-page patent citations to scientific articles. Strategic Management Journal, 41(9):1572-1594, 2020. 


\section{Acknowledgments}

Julia Comeau, Alejandro Fernandez, Haylee Ham, Jaeho Kim, Yanuo Zhou, Simon Neumeyer, and Emmanouil Vachlas provided invaluable research assistance. We thank audiences at the AEA, Barcelona GSE, BAIC, MIT, HBS, IESE, KU Leuven, Madrid Work \& Occupations, and Queen's University, along with Colleen Cunningham, Waverly Ding, Kirstie Ferreira, Dan Gross, Sharique Hasan, Josh Krieger, Matt Marx, Kyle Myers, Ramana Nanda, Debora Spar, and Ariel Stern for helpful feedback and suggestions. Funding: This project has received funding from Harvard Business School and also from the European Union's Horizon 2020 research and innovation programme under the Marie SkłodowskaCurie grant agreement No. 799330. Author contributions: All authors contributed equally. Competing interests: The authors have no known competing interests. Data and materials availability: All data and code are available in the Harvard Dataverse at https://doi.org/10.7910/DVN/V8NJUV

\section{Supplementary Materials}

https://doi.org/10.7910/DVN/V8NJUV

Supplementary Text S1 to S16

Tables S1 to S50

Figures $\mathrm{S} 1$ to $\mathrm{S} 8$

References (35-52) 


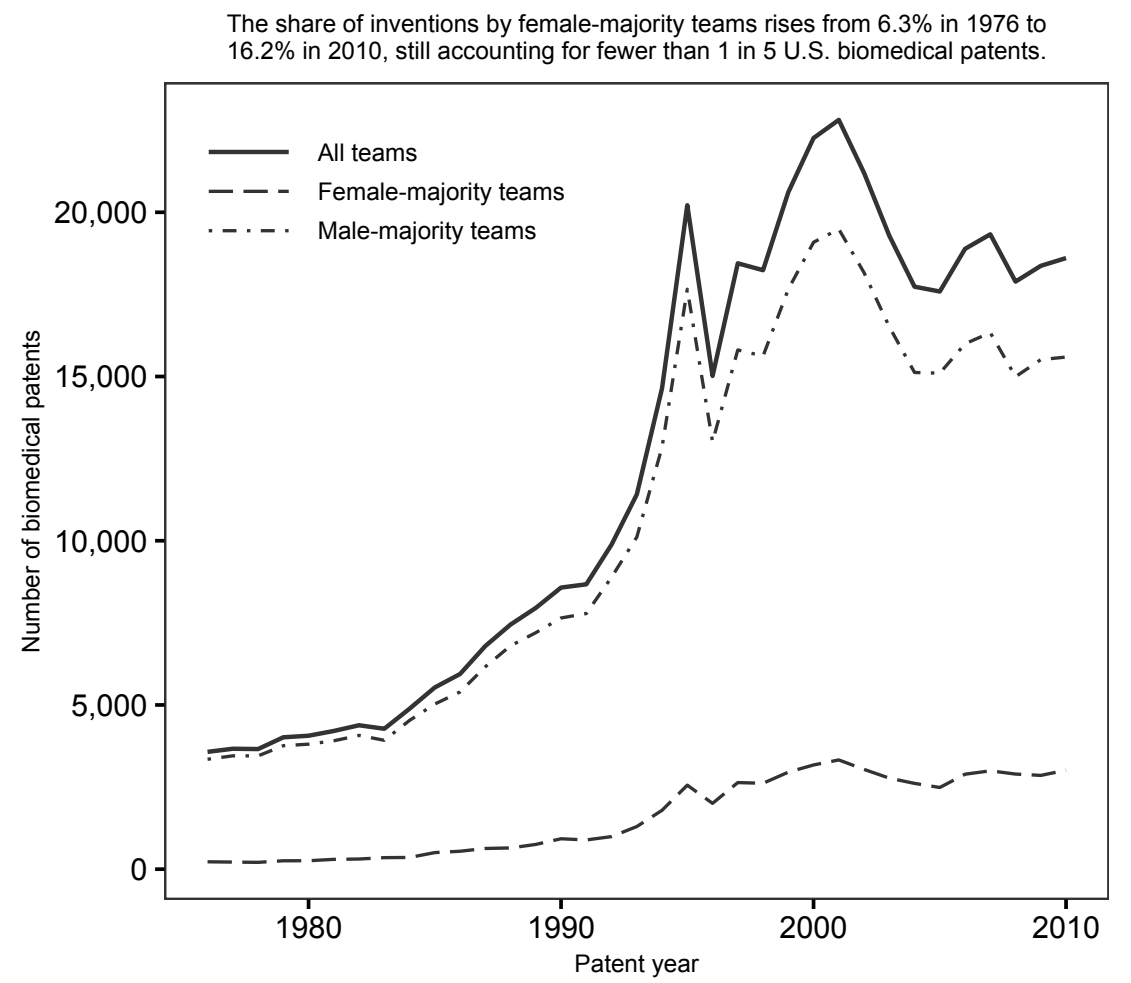

Figure 1: Total number of U.S. biomedical patents over time along with the number of patents with majority female ( $\geq 50 \%$ women) and majority male ( $>50 \%$ men) inventor teams. Includes 430,060 patents from 1976 through 2010. 


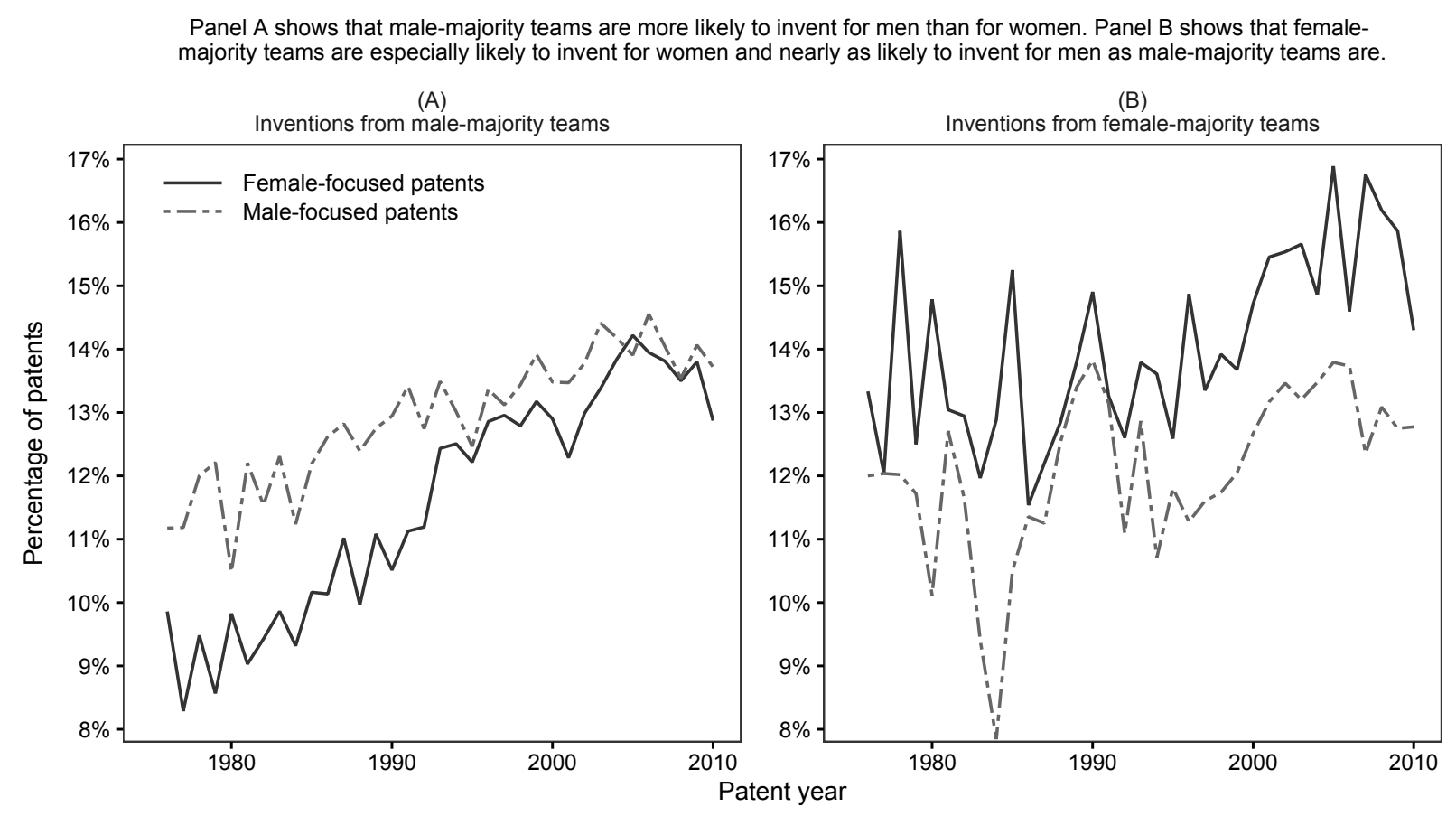

Figure 2: Percentage of U.S. biomedical patents that are male-focused and female-focused broken out by the gender composition of the inventor team. Panel A shows the percentages for patents with majority male teams ( $>50 \%$ men) and Panel B shows patents with majority female teams ( $\geq 50 \%$ women). 

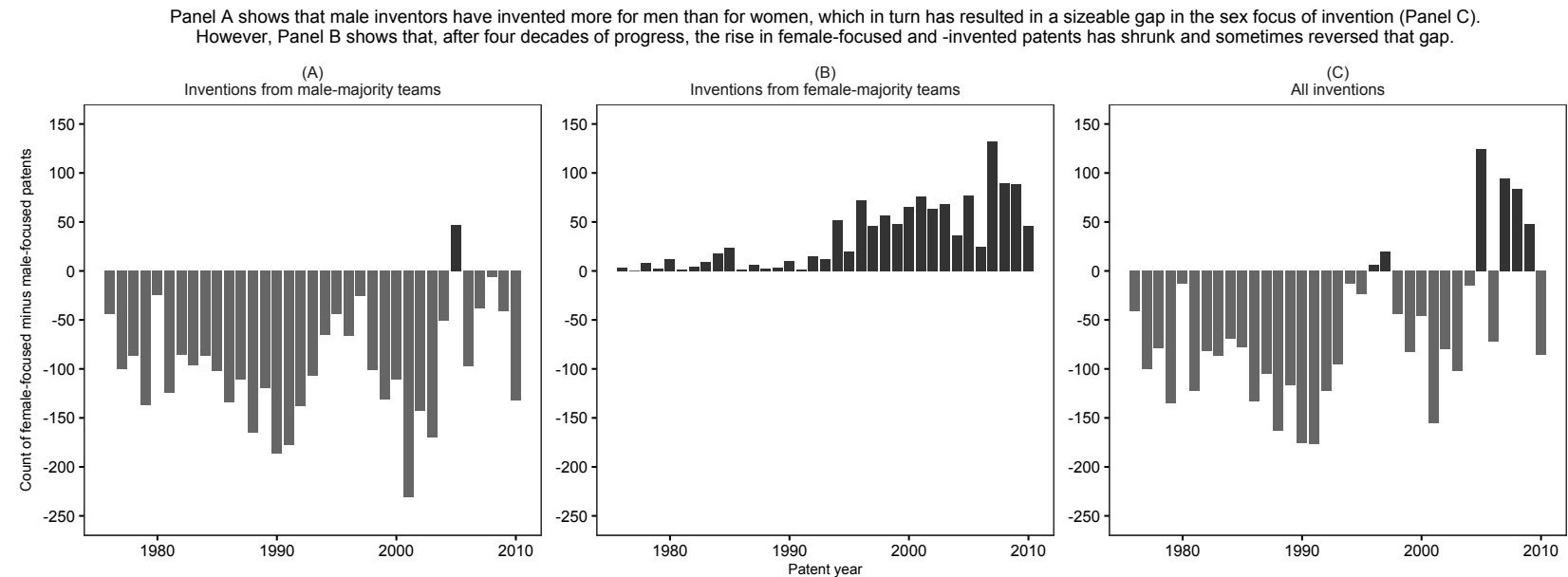

Figure 3: The number of female- minus male-focused patents over time. The first row is the gap for all biomedical patents. The second row is the gap for patents with male-majority teams $(>50 \%$ men). The third row is the gap for patents with female-majority teams ( $\geq 50 \%$ women). 
Patents with more women are more likely to be female-focused both within and across research areas. The same pattern holds for potentially patentable research.
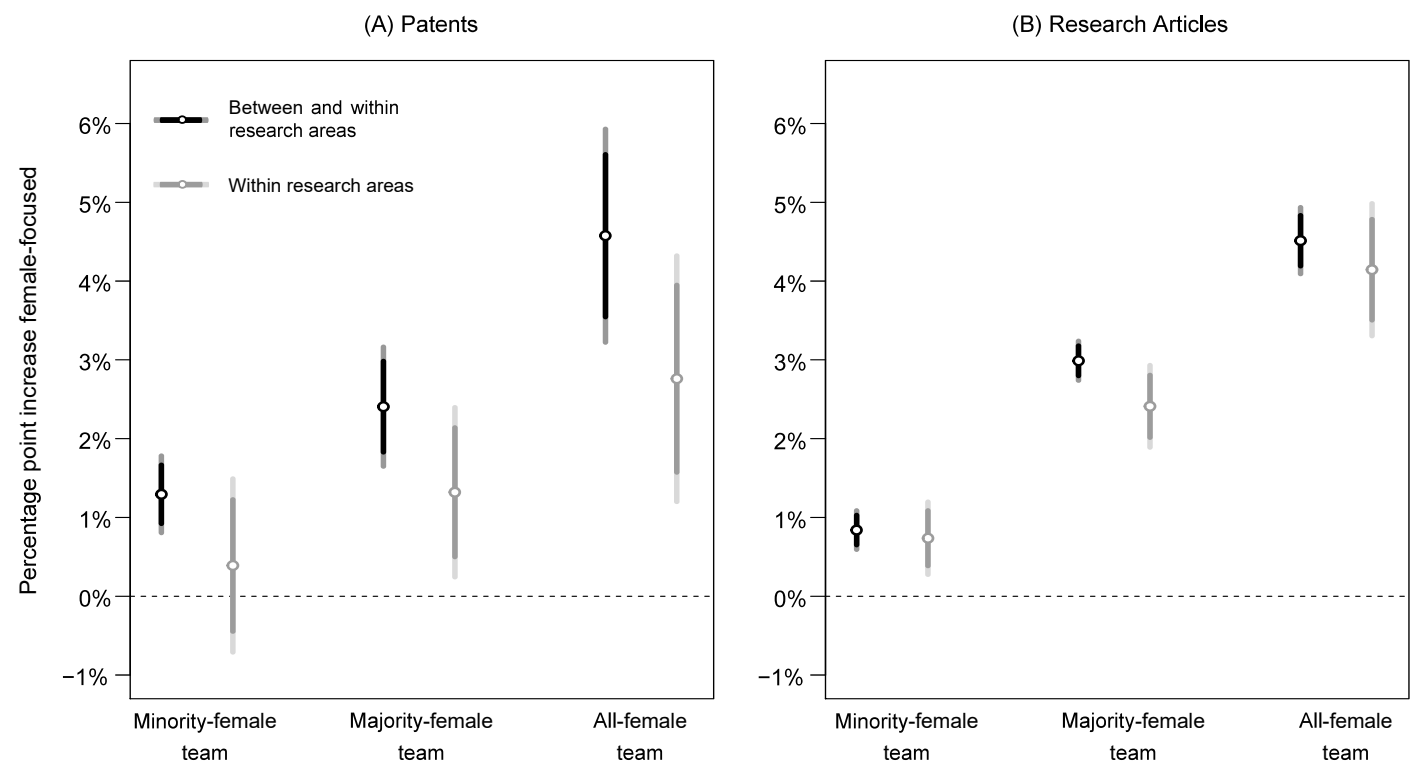

Figure 4: Both panels show the increased chance, in percentage points, that a female-invented patent or research article is female-focused, compared to a patent or article from an all-male invention team. The bars show robust $95 \%$ and $99 \%$ confidence intervals. Panel A reports estimates using the patent data. Black estimates come from a model fit using OLS with year $\times$ subcategory and year $\times$ team-size fixed effects. The gray estimates are from models that match teams with female inventors to all-male teams using the patent's year, subcategory, team-size, and disease focus. The full sample includes 430,060 patents; the largest matched sample includes 45,751 patents. Panel B reports estimates using the research-article data. Black estimates are from models fit using OLS with year $\times$ journal and year $\times$ team-size fixed effects. The gray estimates are similar but also match teams with female inventors to all-male teams using the patent's year, team-size, and disease focus. The full sample includes 2,062,695 articles published in the 1,000 journals in PubMed with the highest commercialization impact factors from 2002 through 2020; the largest matched sample includes 119,650 papers. 


\section{List of Tables}

S-1 Estimates showing the percentage of patents classified as female-focused by the MTI algorithm that are classified as female-focused by trained MeSH indexers. 95\% Confidence intervals in brackets. Top left shows recall, bottom left the false negative rate, bottom right the precision, and top right the false positive rate. . . . . 58

S-2 Estimates showing the percentage of patents classified as male-focused by the MTI algorithm that are classified as male-focused by trained MeSH indexers. $95 \%$ Confidence intervals in brackets. Top left shows recall, bottom left the false negative rate, bottom right the precision, and top right the false positive rate. . . . . . . . . 59

S-3 Precision and recall for whether a patent is female-focused, split by the gender composition of the inventor team. 95\% Confidence intervals in brackets. In each table the top left shows recall, bottom left the false negative rate, bottom right the precision, and top right the false positive rate. . . . . . . . . .

S-4 Precision and recall for whether a patent is male-focused, split by the gender composition of the inventor team. 95\% Confidence intervals in brackets. In each table the top left shows recall, bottom left the false negative rate, bottom right the precision, and top right the false positive rate. . . . . . . . . . . . . 60

S-5 The 100 words most associated with patents tagged with the "Female" check tag. . . 61

S-6 The 100 words most associated with patents tagged with the "Male" check tag. . . . 62

S-7 Percentage of patents classified by the authors as focusing on the needs of women and men, by MeSH sex tag . . . . . . . . . . . . . . . 63

S-8 Patents tagged as female by the MTI address diseases with higher female incidence counts; male-tagged patents address diseases with higher male incidence . . . . . . . 64

S-9 List of patents with our female-only trial prediction measure . . . . . . . . . . 65

S-10 List of patents with our male-only trial prediction measure . . . . . . . . . . 66

S-11 Patents tagged as female by the MTI are more likely to describe inventions that would require a female-only clinical trial; patents tagged as male are more likely to require a male-only clinical trial . . . . . . . . . . . . . . . . . 67

S-12 Gender-identification success rates, by team size and geographic location . . . . . 68

S-13 Patent regressions corresponding to Figure 4A . . . . . . . . . . . . . 69

S-14 Matched patent regressions corresponding to Figure 4A (minority-Female Teams) . . 70

S-15 Matched patent regressions corresponding to Figure 4A (majority-female teams) . . 71

S-16 Matched patent regressions corresponding to Figure 4A (all-female teams) $\ldots \ldots$. . 72

S-17 Do female inventors invent for men? . . . . . . . . . . . . . . . . 73

S-18 Do female inventors invent for men? Exact matching results (minority-female teams) 74

S-19 Do female inventors invent for men? Exact matching results (majority-female teams) 75

S-20 Do female inventors invent for men? Exact matching results (all-female teams) . . . 76

S-21 Patent regressions hold when using logistic regression or focusing on female-only

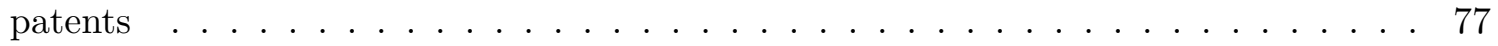

S-22 Patent regressions hold when we feed more or less patent text to the MTI algorithm 78

S-23 Patent regressions hold when we restrict our sample to patents tagged as focusing on "humans" or by excluding patents tagged as focusing on "animals" . . . . . . . . 79

S-24 Women are more likely to invent for women when using alternative measures of patent's sex focus . . . . . . . . . . . . . . . . 80

S-25 The findings presented in Figure 4A, Figure S-5, and Table S-13 replicate when we use the estimated female-only trial probability in place of the female check tag . . . 
S-26 The findings presented in Figure 4A, Figure S-5, and Table S-14 replicate when we use the estimated female-only trial probability in place of the female check tag . . . 82

S-27 The findings presented in Figure 4A, Figure S-5, and Table S-15 replicate when we use the estimated female-only trial probability in place of the female check tag . . . 83

S-28 The findings presented in Figure 4A, Figure S-5, and Table S-16 replicate when we use the estimated female-only trial probability in place of the female check tag . . . 84

S-29 The findings hold when we match on Disease-Subcategory and Year-Disease-Subcategory 85

S-30 Patent regressions showing our findings hold when we include more or fewer disease

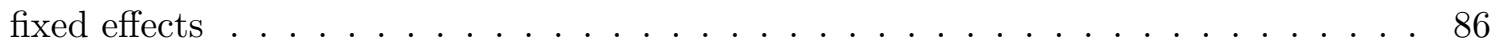

S-31 Patent regressions with double selection lasso show similar results . . . . . . . . . 87

S-32 Publication regressions corresponding to Figure 4B . . . . . . . . . . . . 87

S-33 The 100 words most associated with publications tagged with the "Female" check tag 88

S-34 The 100 words most associated with publications tagged with the "Male" check tag . 89

S-35 Papers tagged as female address diseases with higher female incidence counts; male tagged papers address diseases with higher male incidence . . . . . . . . . . . 9 90

S-36 List of publications with our female-only-trial prediction measure . . . . . . . . . . . 91

S-37 List of publications with our male-only-trial prediction measure . . . . . . . . . . 92

S-38 Papers tagged as female are more likely to describe ideas that would require a femaleonly clinical trial; papers tagged as male are more likely to require a male-only clinical

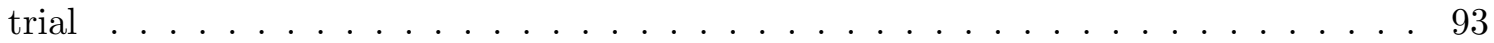

S-39 Publications by women are not more likely to focus on the medical needs of men . . 94

S-40 Women are more likely to discover for women regardless of the Journal Commercialization Impact Factor (JCIF) of the journal where the article is published . . . . . . 95

S-41 The findings in Figure 4B hold when match on journal instead of disease area . . . . 96

S-42 Publication regressions testing heterogeneity by time-period and authorship order . . 97

S-43 Women are more likely to discover for women when using alternative measures of a paper's sex focus . . . . . . . . . . . . . . . . . . . . 98

S-44 The findings in Figure 4B of paper hold when we instead use our clinical-trial measure of a paper's sex focus . . . . . . . . . . . . . . . . . . 99

S-45 Patents by women are more likely to commercialize female focused papers . . . . . 100

S-46 The number of female-focused and male-focused patents by whether the inventors are majority women (greater than or equal to 50\%) or majority men (strictly greater than $50 \%) \ldots \ldots \ldots \ldots \ldots 1$

S-47 Summary statistics for our patent data . . . . . . . . . . . . . . 101

S-48 Summary statistics for our publication data . . . . . . . . . . . . . . . 102

S-49 Summary statistics for our patent data, 2002-2010 . . . . . . . . . . . . 102

S-50 Summary statistics for our publication data, 2002-2010 . . . . . . . . . . . . 102

\section{List of Figures}

S-1 Screenshot from the web app used by human medical indexers to evaluate our algorithm. The human, female, and male check tags were always displayed at the top, irrespective of whether the MTI classified the patent as matching these tags. . . . . 103

S-2 Screenshot from the web app used by human medical indexers to evaluate our algorithm. The top $40 \mathrm{MeSH}$ tags that the MTI assigned to the patent were displayed. The indexers could delete tags they thought did not apply to the patent. . . . . . . 104 
S-3 Screenshot from the web app used by human medical indexers to evaluate our algorithm. At the bottom of the screen the indexers could add MeSH terms that they thought the MTI had missed. The text box would auto-complete the terms they typed, to ensure they were adding a valid MeSH tag. . . . . . . . . . . . . . . 105

S-4 These estimates show the increased chance, in percentage points, that a femaleinvented patent is male-focused, compared to a patent from an all-male invention team. Black estimates are from models are estimated using OLS with year $\times$ subcategory and year $\times$ team-size fixed effects. The blue estimates are from models that match teams with female inventors to all male teams using the patent's year, subcategory, team size, and disease focus. The bars show robust $95 \%$ and $99 \%$ confidence intervals. The full sample includes 430,060 patents; the largest matched sample includes 45,751 patents. . . . . . . . . . . . . . . . . . . . . 106

S-5 These estimates show the increased chance, in percentage points, that a femaleinvented patent is female-focused, compared to a patent from an all-male invention team. Black estimates come from models that are estimated using OLS with year $\times$ subcategory and year $\times$ team-size fixed effects. The blue estimates are from models that match teams with female inventors to all-male teams using the patent's year, subcategory, team size, and disease focus. The bars show robust $95 \%$ and $99 \%$ confidence intervals. The full sample includes 430,060 patents; the largest matched sample includes 45,751 patents. . . . . . . . . . . . . . . . . 107

S-6 Annual percentage of U.S. $d r u g$ biomedical patents by inventor-team gender and the sex focus of the patent. Female-majority teams are those with $50 \%$ or more female inventors on the patent. Data on inventor gender comes from a dictionary-match analysis of inventor names from PatentsView. Data on the sex focus (if any) of patents comes from passing patent text through the National Library of Medicine's Medical Text Indexer. A patent is coded male- or female-focused if the MTI returns "Male" or "Female" as one of its top Medical Subject Headings, or "MeSH," terms.

S-7 Annual percentage of U.S. surgery and instrument biomedical patents by inventorteam gender and the sex focus of the patent. Female-majority teams are those with $50 \%$ or more women inventors on the patent. Data on inventor gender comes from a dictionary-match analysis of inventor names from PatentsView. Data on the sex focus (if any) of patents comes from passing patent text through the National Library of Medicine's Medical Text Indexer. A patent is coded male- or female-focused if the MTI returns "Male" or "Female" as one of its top Medical Subject Headings, or "MeSH," terms. . . . . . . . . . . . . . . . . . . . . . . 109

S-8 Estimated number of female-focused inventions that are lost each year through the under-representation of women in invention. . . . . . . . . . . . . 110 


\section{Supplementary Materials}

\section{Overview of Materials and Methods and Supplementary Text}

- S-1 Building the patent dataset and assigning MeSH terms to patents with the MTI algorithm

- S-2 Validating that the MTI algorithm works with patent text

- S-3 Measuring and validating the sex-focus of inventions

- S-4 Inventor gender classification

- S-5 Regression tables corresponding to Figure 4A

- S-6 Female inventors are neither more nor less likely to invent for men

- S-7 Robustness to alternative modeling assumptions, text processing, and dependent variables

- S-8 Accounting for selection with additional fixed effects and double-selection lasso methods

- S-9 Heterogeneity in the female-inventor invention link

- S-10 Building the PubMed dataset

- S-11 Regression tables corresponding to Figure 4B

- S-12 PubMed publication-analysis robustness checks

- S-13 Back-of-the-envelope calculations of lost female-focused patents

- S-14 Summary statistics

- S-15 Four example patents along with MTI assigned MeSH terms

- S-16 MeSH-GBD Crosswalk 


\section{S-1 Building the patent dataset and assigning MeSH terms to patents with the MTI algorithm}

Our patent data come from the PatentsView and NBER patent data files. We focus on the universe of "Drugs \& Medical" patents (Patent category 3) filed from 1976 through 2010. As of writing, the NBER patent data covers patents granted through 2015. Since approval can take years, the dataset only includes patents filed in 2014 that were approved within one year, within two years for 2013, and so on. The vast majority of patents are approved within four years. Thus we drop all patents filed after 2010 to ensure that our sample reflects the population of eventually granted patents for any given filing year. Our results are essentially unchanged if we include the post-2010 patent data. After this cleaning, we have 441,580 inventions.

To classify patents by the disease or condition they target, we draw on the National Library of Medicine's Medical Text Indexer $(35,36)$, which generates Medical Subject Headings, or MeSH terms, for a given body of text. We passed the first 2,500 characters of each patent's title, abstract, and summary through the National Library of Medicine's Medical Text Indexer (MTI). According to its documentation, the MTI allows up to 10,000 characters of input text, but we found that the indexer was much more likely to "choke" and crash when the input text approached this length. After trial and error, we found that limiting the text to 2,500 characters produced substantially fewer failed classification runs. ("Failed" in the sense that the indexer ran without crashing, not in the sense that it failed to classify a patent's text.) Using just the title and abstract yields similar findings. We use the default MTI settings for non-Medline text, and we output MeSH terms from the 2018 edition.

Because the MTI's purpose is to help medical researchers discover related work, its default results are "greedy," suggesting hundreds of potentially related (but low-scored) terms. We therefore discard the least-related MeSH terms with scores of 0 or -1 . We then use the remaining MeSH terms returned by the MTI in our analysis. Our findings are unchanged if we restrict the MeSH terms we analyze to the top 10 or top 25 terms returned by the MTI algorithm. See S14 for summary statistics. 


\section{S-2 Validating that the MTI algorithm works with patent text}

A potential concern with using the MTI to assign sex focus to patents is that its algorithm was not designed with this specific task in mind. Rather, it was designed to classify journal articles, to aid researchers. This poses three risks for this study. The first is that the MTI might simply produce extremely noisy estimates of a patent's sex focus that are unrelated to how a human reviewer might assign MeSH tags. The second and more serious is that, for whatever reason, the MTI could assign one gender tag or the other at significantly higher rates when confronted with this novel text corpus. This would introduce bias, rather than just noise, into our models. Finally, the female and male MeSH tags in and of themselves might not actually reflect whether a patent is more or less focused on the medical needs of women or men, irrespective of wether they are assigned by a human reviewer or an algorithm. Here we address these first two concerns. The next section addresses the third concern, about construct validity.

To address whether the MTI assigns tags as a human indexer would, we hired two indexers who have worked on assigning MeSH terms to biomedical research articles. Both have worked as indexers for a decade. We presented each of them with 480 randomly selected patent texts and asked them to assign MeSH terms as though they were evaluating an article, in their usual manner. Neither indexer was informed that we were studying whether female inventors invented for women. They were informed that we were studying the classification accuracy of the MTI algorithm on patents. Each evaluated patents independently.

To aid in this evaluation, we developed a custom web app for evaluating the MeSH terms applied to a patent. The indexers were shown the patent's title and the text that we fed to the MTI. They were not shown the names or gender of the inventors. Nor were they given a link to the patent. This ensured that we could evaluate the MTI's accuracy on exactly the same data that we fed to the MTI.

Figures S-1, S-2, and S-3 are screenshots from the web app, showing the patent's text and the MeSH terms that the MTI had assigned it. The indexers were instructed to click on any MeSH terms they thought were inappropriately applied to the patent. At the bottom of the screen, they were instructed to add any MeSH terms that they thought the MTI had overlooked. MeSH indexers regularly use the MeSH-on-demand tool, which uses the MTI to generate suggested MeSH terms, 
as a starting point for their work indexing biomedical research articles.

Finally, we listed three check tags that are always displayed at the top of the app. These tags are "Human," "Female," and "Male." If the tag was not selected by the algorithm, the indexer could click on it to apply it to the patent. When indexing biomedical articles, check tags are always displayed, so that indexers make sure to either select or not select the check tags. We again note that the female and male check tags have specific definitions in the MeSH ontology. Indexers are supposed to apply the "Female" check when the text covers "female organs, diseases, physiologic processes, genetics, etc." Indexers are supposed to apply the "Male" check when the text covers "male organs, diseases, physiologic processes, genetics, etc."

Each indexer was asked to evaluate 480 randomly selected patents. Since female-invented and female/male-focused patents are relatively rare, and to ensure that we could evaluate errors in different sub-samples of our data, we stratified the sample on whether the patent is from a femalemajority team ( $50 \%$ or more female inventors), is female-tagged, is male-tagged, and is from before 1996, and then by its subcategory. In each of the resulting 32 strata we randomly sample 15 patents for validation. Since we use stratified oversampling, all the cross-tabulations below include sampling weights to give population-weighted estimates for the algorithm accuracy across our entire sample. We classify a patent as female- or male-focused when both indexers agree that it is. The indexers could skip patents that they thought they lacked expertise to evaluate. Only 5 patents were skipped, yielding 475 patents evaluated by both indexers.

In Table S-1 we show the recall/precision rates for whether a patent is female-focused. The rows indicate whether the human indexers tagged the patent as covering "female organs, diseases, physiologic processes, genetics, etc." The columns indicate whether the MTI algorithm tagged the patent as female-focused. Recall, or one minus the false-negative rate, is the estimate in the top left; the false-negative rate is the estimate on the bottom left. Precision, or one minus the false positive rate, is the estimate in the bottom right; the false positive rate is in the top right. Table S-1 shows that the MTI has surprisingly good precision, about 93\%, for whether a patent is female-focused. Recall is less strong at $65 \%$ but, as we discuss below, this is par for the course for indexing algorithms. Further, the $F_{1}$ score is 0.77 , which compares favorably to estimates of the MTI's $F_{1}$ on PubMed articles, 0.74, for the female-check tag as of 2016 (37).

Table S-2 shows the recall/precision rates for whether a patent is male-focused. The estimates 
therein do not statistically differ from those in Table S-1. The MTI appears to do as well at classifying patents as male-focused as it does at classifying them as female-focused, with an $F_{1}$ score of 0.78. Again, this is consistent with past work showing the that the MTI, when analyzing PubMed articles, has an $F_{1}$ of 0.71 (37). Overall, these results imply that the indexers thought that many more patents should have been tagged as male or female than the MTI did. Far from being likely to over-assign sex tags, the MTI indexer appears conservative.

We next check whether the algorithm is more or less accurate for male- versus female-majority teams. Table S-3 presents recall/precision rates for female-majority teams (50\% or more female inventors) and for male-majority teams (strictly less than 50\% women). Precision is slightly higher for female-majority teams, but the difference is not significant. Recall is nearly identical. If anything, the MTI is somewhat more likely to falsely classify a patent as female-focused when the team is majority-male. This would bias our estimates of the female inventor-invention link downward.

Table S-4 replicates Table S-3, but for whether the patent is male-focused. We again find no significant differences across team composition. It does not appear that our findings are driven by biases in the MTI algorithm. In additional analyses, we also find that the algorithm's precision and recall is the same for patents before and after 1996 (This is halfway through the sample; we find no sensitivity to the year in which we break the sample). The only meaningful difference we find is between drug and surgery patents. The MTI's recall rates are just above $70 \%$ for drugs, compared to about $55 \%$ for surgery patents.

It is worth comparing these these precisions/recall rates against other benchmarks of the MTI. Mork et al. (20) find that, when indexing biomedical research articles, the MTI's recall and precision rates had converged to about $60 \%$ by 2014 . In a more recent article Rae et al. (38) compare the performance of the MTI against a state-of-the-art convolutional-neural-net algorithm. While these methods have a lot of promise, their present performance gains are modest, at about $3 \%$. Finally, the most recent BioASQ Challenge, in which teams compete to develop algorithms that better index medical journal articles, the best submission in the final round had a precision of just over $70 \%$ and a recall of $59 \%$, compared to the MTI's rates of $60 \%$. Finally, as mentioned above, DemnerFushman and Mork (37) find that the MTI as of 2016 has $F_{1}$ scores for the male and female check tags when analyzing PubMed text that are very similar to what we find when applying the MTI 
to biomedical patent text. The MTI appears to generalize well for our purposes.

To summarize, our independent validation of the MTI using professional human indexers suggests that the MTI produces somewhat noisy but unbiased estimates of whether a patent would be assigned the female or male tag by a human indexer.

Before turning to the next section, which validates that patents tagged with the female check tag are in fact more focused on the medical needs of women, it is worth noting that, by relying on the MTI's sex tags, we implicitly treat a patent's gender focus as focusing on neither sex, just women, just men, or both men and women. This is a crude simplifying assumption. Patents likely address the sexes on a continuum. The next section tackles some of these concerns, though we hope future work continues to build more nuanced measures of a patent's sex focus that take into account the non-binary nature of both sex and gender. 


\section{S-3 Measuring and validating the sex focus of inventions}

Are inventions tagged with the "Female" MeSH term more likely to meaningfully focus on the medical needs of women? Do "male"-tagged inventions disproportionately benefit men? Appendix S2 demonstrates that the MTI algorithm tags patents as female or male similarly to how trained human medical indexers do. However, indexers are encouraged to be relatively aggressive in applying the female and male MeSH check tags. For example, the female tag is supposed to be applied when a paper focuses on "female organs, diseases, physiologic processes, genetics, etc." but can also be applied when the paper merely describes collecting samples from "women," even if the research doesn't especially benefit women. This raises an important construct-validity concern: do the female/male check tags measure differences in an invention's likelihood of focusing on the medical needs of women or men?

Measuring whether an invention "focuses on the medical needs of women" is non-trivial. On the one hand, there are cases where the focus on women is obvious. For example, an invention diagnosing whether ovarian cysts are cancerous will clearly benefit women. Indeed, the entrepreneur Surbhi Sarna eventually built just such an invention after suffering from a ovarian cyst as a teenager (14). On the other hand, many inventions benefit women in less obvious ways. For example, Patricia Bath, the first black female doctor to receive a medical patent, invented a more precise treatment for cataracts. Her interest in the subject grew from her concern that African Americans, and especially black women, were almost an order of magnitude more likely to suffer from Glaucoma and blindness than white men (16). Thus, while the invention may not explicitly call out female anatomy, by focusing on a condition that especially impacts women and black patients, the invention benefits both groups. Finally, an invention might target diseases that equally impact both men and women, but the development of the drug or intervention might have relied on all-male samples and/or failed to do sex analyses that could reveal adverse reactions amongst women $(12,23)$. Thus, an invention that is tested on samples that include women or that runs a sex analysis might not be explicitly focused on the needs of women, but these inventions might be more likely to benefit women or at least not cause additional harm. Indeed, prior work has used the presence of sex-specific MeSH terms as a proxy for whether academic research engages in sex reporting and analysis (39). Thus, while inventions no doubt vary in who benefits from them, these examples highlight the complex 
ways in which an invention can be more or less female/male focused.

Given this complexity, we use four approaches to validate that female-tagged patents are, in fact, much more likely to focus on diseases, topics, and conditions that disproportionately impact women. Across our four measures we find strong evidence that patents tagged as "female" by the MTI are much more likely to benefit women; similarly, patents tagged as "male" by the MTI are much more likely to benefit men. Below we briefly summarize each approach before describing them in detail.

Our first approach uses relatively simple text-analysis tools to identify the words most associated with female/male tagged patents, as compared to non-sex-tagged patents. Consistent with the idea that the female MeSH term reflects "female organs, diseases, physiologic processes, genetics, etc.," we find that female-tagged patents are 403 times more likely to use the word "menopause," 245 times more likely to use the word "cervix," and 7.3 times more likely to use the word "fibromyalgia" than non-female-tagged patents (four in five fibromyalgia sufferers are women). Male-tagged patents are 274 times more likely to use "erectile," 146 times more likely to use "prostate," and 2.2 times more likely to use "parkinson" (two thirds of Parkinson's cases occur in men). The final two examples in each list are particularly interesting as they, along with a number of other terms described below, suggest that the male/female MeSH tags capture both obvious and more subtle differences in who is likely to benefit from an invention.

Our second approach relies on human review to evaluate whether a patent is female or male focused, allowing us to check if the aforementioned differences in word usage truly reflect differences in the population of who is most likely to benefit from an invention. Specifically, we sampled 400 patents and then searched for research articles reviewing sex differences in the incidence of the primary condition addressed by the invention. We find that about $60 \%$ of patents tagged as female/male are focused on conditions that disproportionately affect women/men; this rate is the same for patents invented by men and women. The majority of sex-tagged patents are sex-focused. Though many are not, such measurement error biases findings towards zero, implying our estimates of the female inventor-invention link are conservative. Further, patents without a sex tag exhibit female and male sex-focus only $5 \%$ of the time, implying that a patent tagged with the female or male MeSH tag is an order of magnitude more likely to address medical conditions that particularly impact women or men. When we take these misclassification rates into account, we still find that 
the overall percentage of female focused patents hovers around $12 \%$.

Our third approach builds on the spirit of the second approach but uses disease-by-sex incidence data to estimate how many more women stand to benefit from an invention when it is tagged as female. We find that, when a patent is tagged as female, it addresses diseases that afflict $130 \%$ more women than when it is not; when it is tagged as male, it addresses diseases that afflict $73 \%$ more men than when it is not. On average, female- or male-tagged patents benefit more women or men than non-sex-tagged patents.

Our fourth and final approach measures a patent's female focus by estimating whether the invention is more likely to be clinically evaluated in a trial that only recruits women. Using enrollment restriction and the text data from of over 350,000 clinical trials from 1994 to 2020, coupled with a word-embedding model trained on our patent and PubMed data, we build an algorithm that takes in text describing an intervention, procedure, or drug and then estimates the probability that it would be tested on only women or only men. We then apply this algorithm to our patent data to estimate the probability an invention would require a single-sex clinical trial. We find that female- and male-tagged patents are roughly $400 \%$ more likely to describe inventions that would be be evaluated by a female- or male-only clinical trials.

To summarize: across our four approaches, we find strong evidence that the sex MeSH tags capture meaningful differences in which sex is most likely to benefit from an invention. Our disease incidence and estimated female/male-only-trial measures yield two alternative dependent variables that we use to directly test if female inventors are, in fact, more likely to invent for women. In Appendix S7 we show that our results hold when we use either of these measures in lieu of our primary female MeSH tag measure.

\section{S-3.1 Female/male tagged patents use more words associated with female/male "organs, diseases, physiologic processes, genetics, etc."}

Our first approach tests that the words used to describe female-tagged inventions are in fact female focused, and that male-focused words are used for male-tagged inventions. We do so by identifying the 100 words most likely to appear in female-tagged versus non-female-tagged patents. Unfortunately, using raw odds ratios (or similar TF-IDF measures) often yields a noisy word list, because infrequently used words can, by chance, end up over-represented amongst female- or male-tagged 
patents. To address this problem, we rely on a weighted log-odds-ratio to generate a z-score for how "over-represented" each word is (40). We then keep words with a score over 3.891 (i.e., $p<0.0001$ ) to ensure that observed differences are not artifacts of chance (lower z-score cutoffs yield similar patterns). To ensure that the examples reflect non-esoteric conditions and concerns, we further restrict our sample to words that occur at least 100 times in our 60-million-word patent text corpus, though again other cutoffs yield similar patterns. Here we analyze the same snippets of text we use to produce our primary MTI MeSH tag measures, though yet again these patterns hold if we analyze different quantities of patent text.

Table S-5 shows the 100 words that are most over-represented amongst female-tagged patents compared to non-female-tagged patents. Alongside each word we report the raw odds-ratio ${ }^{1}$ and the weighted z-score. The list of words clearly skews female-related. The top words include "gynecologic" (with odds 2,113 times greater in female-tagged than non-female-tagged patents), "woman" $(492 \times)$, "breastpumps" $(552 \times)$, "vaginal" $(257 \times)$, "mammogram" $(145 \times)$, "pregnancy" $(78 \times)$, and "endometriosis" $(73 \times)$. Some of the errors are easily explainable: for example, the most overrepresented term is "cows." While not necessarily relevant for female humans, "cows" does capture patents relevant for female bovines. Indeed, the table reveals that female-tagged inventions often pick up inventions focused on female animals ("sows," "hens") instead of female humans. While some of these patents discuss interventions designed for humans but developed on these animals, others are clearly veterinary. In Table S-23, discussed in more detail in Section S7, we show that our findings hold when we exclude patents that discuss animals.

Table S-6 shows the 100 words that are most over-represented amongst male-tagged patents. The terms include "prostatectomy" $(3,637 \times)$, "cavernosa" $(2,008 \times)$, "erection" $(506 \times)$, "testicular" $(32 \times)$, and "male" $(22 \times)$. We again see animals listed, with "bull" having an odds-ratio of 287 . Consistent with the idea that patents focused on sexual intercourse might be more likely to be tagged as female and male, we see that "clitoris" is 20 times more likely in male- than non-maletagged patents. However, for female-tagged patents, the same odds ratio is 474, more than 20 times larger again.

While these lists focus on the most over-represented words, it also appears that that sex-tagged

\footnotetext{
${ }^{1}$ We add 1 to the denominator and numerator to avoid divide-by-zero concerns when constructing the odds and
} odds-ratios. This transformation does not meaningfully alter our pattern of findings. 
patents are also more likely to use words that describe conditions that disproportionately impact women/men, but that are not exclusively linked to either sex. For example, drug abuse is roughly two-times more common in men than in women. Consistent with this differential impact, we find that the word "cocaine" occurs $2.7(z=5.0)$ times more often in male-tagged patents and is actually less likely to occur in female-tagged patents $(0.8 \times, z=-0.4)$. Similarly, Parkinson's disease affects men at twice the rate it affects women, and we find an odds ratio of $2.2(z=8.0)$ for male-tagged patents versus $1.2(z=2.1)$ for female-tagged patents. In terms of diseases that disproportionately impact women, we find that fibromyalgia, for which women account for over $80 \%$ of cases, has an odds ratio of $7.3(z=7.1)$ for female-tagged patents and an odds ratio of $3.3(z=3.9)$ for maletagged patents. Lupus, which is about nine times more common in women than in men, has an odds ratio of $2.8(z=5.9)$ for female-tagged versus $1.9(z=3.6)$ for male-tagged patents.

We find strong evidence that the words most over-represented in male-/female-tagged patents are words that disproportionately relate to men/women's health.

\section{S-3.2 Patents tagged as female/male by the MTI are more likely to address conditions that disproportionately impact women/men}

Our second approach validates the sex focus of the check tags by reviewing 400 randomly sampled patents: 100 with no sex check tags, 100 with only the female tag, 100 with the male tag, and 100 with both sex tags. For each patent we classified the invention as "female focused" if it addressed conditions and diseases that disproportionately impact women. To measure whether a patent disproportionately benefits women, we conducted a literature search on the diseases and conditions the patent addresses. Focusing on review articles, we checked whether prior research has highlighted meaningful differences in the incidence of the disease or condition by sex. For example, as discussed above, lupus impacts both men and women, but nine out of ten patients with lupus are women. If an invention primarily addressed lupus then we would then classify the patent as focused on the health needs of women. Conversely, "Sleep Apnea" sufferers are three times more likely to be men than women. Thus, patents addressing "Sleep Apnea" would be classified as male focused. Patents that address "hip replacement" would be marked as neither male- nor female-focused, since the procedure occurs at essentially the same rate for both sexes. Patents that describe upstream inventions, like new ways of coating a pill, would also not be marked as male or female focused. 
In the case of sex-based anatomical differences (e.g., patents focused on "testicles" or "fallopian tubes") we would skip the review and simply mark the patent as female/male focused based on the anatomical difference. Similarly, if the patent explicitly stated that it was targeting conditions that disproportionately impact men or women, we would mark it as such. Finally, in cases where a patent clearly indicates that it treats multiple conditions that disproportionately impact either sex (e.g., a drug that can treat both "PMS" and "male sexual dysfunction"), we mark the patent as both male and female focused.

Table S-7 shows the percentage of patents focused on the medical needs of women, men, or both, separated by the patents's MTI-assigned MeSH tags. When a patent is neither assigned the male nor the female tag it addresses majority-female medical needs $5 \%$ of the time and majority-male medical needs $6 \%$ of the time. In contrast, when the patent is tagged as male, it addresses topics that disproportionately impact men $57 \%$ of the time; female-tagged patents address topics that disproportionately impact women $62 \%$ of the time. When tagged with both sex tags, the patent is male or female focused roughly $25 \%$ of the time and explicitly address both sexes only $16 \%$ of the time. Of the roughly $40 \%$ of patents that are tagged as female or male, but that are not sex focused, it seems they get the tag because they list sex-specific outcome data or incidentally mention the words "male," "female," "woman," or "man." We also find standard text-analysis errors, such as where the MTI picks up words that are associated with men or women but used in a different context. For example, "sheath" is regularly tagged as male when it should not be, because male-focused patents are much more likely to use the phrase "penile sheath" and thus the word "sheath" than female-focused patents.

Though the samples are small, we also checked if there is any evidence that patents by women are more or less likely to be female focused when tagged with the female MeSH tag. We find no such evidence $(p=0.25)$. Similarly, we find no difference by inventor gender in the probability that a patent is male focused when tagged with male MeSH tags $(p=0.33)$. This lack of bias echoes the findings described in S2, which shows that the MTI is unbiased when compared to how human indexers apply the sex MeSH tags. This unbiased measurement error biases our findings towards zero, implying our estimates of the female inventor-invention link are likely conservative.

When we take into account these misclassification rates, we still find that the overall percentage of female focused patents hovers around $12 \%$. Using these rates, we expect $62 \%$ of the just under 
$13 \%$ of female-tagged patents to be female focused, reducing the number of actually female focused

patents to about $8 \%$. However, the results in Table S-7 suggest that $5 \%$ of non-sex-tagged patents are actually female focused. Given that roughly $80 \%$ of patents are not tagged as male or female, this suggests that there are $4 \%$ more female focused patents that are not tagged as such by the MTI. Putting the two estimates together yields an overall rate of roughly $12 \%$. The same arithmetic holds for male focused patents. Even if the levels were off by a third, the difference between female and male remains unchanged. Therefore the back-of-the-envelope calculations we show in S13 would still suggest that the lack of female inventors might have led of thousands of lost female focused inventions.

To summarize, we find that the majority of patents tagged as female or male by the MTI are, in fact, focused on diseases or conditions that tend to impact women or men. The differences between patents with no sex tags and those tagged as male or female is substantial. Being tagged as male/female increases ten-fold the chance that an invention is male/female focused. Put in terms of evaluation accuracy, patents tagged as female or male have $F_{1}$ scores of just over $73 \%$ when it comes to classifying patents as male or female focused, a rate similar to the MTI indexer's ability to apply the sex MeSH tags as human indexers would (though with flipped precision and recall rates).

Finally, Table S-7 suggests caution when interpreting patents that are assigned both sex tags. It appears that when assigned both tags, the patents are more likely to actually benefit one sex or the other rather, than to target sex-focused conditions that impact both sexes. In our case, since our interest lies in whether a patent is female focused, this distinction is less of a concern. Regardless, in Appendix S7 Table S-21 we show that our findings hold when we mark patents as female focused only if the female check tag is present and the male check tag is not.

\section{S-3.3 Patents tagged as female/male address diseases with higher male/female incidence}

If our second approach marks patents as either female or male focused, our third approach quantifies not just whether a patent disproportionately benefits women/men, but how many more women/men stand to benefit from a female/male tagged invention. To do so, we build a crosswalk from the set of diseases in the MeSH ontology to the disease topics in the Institute of Health Metrics and 
Evaluation's Global Burden of Disease database (GBD). ${ }^{2}$ The GBD database measures yearly country-level incidence by hundreds of disease topics (41). ${ }^{3}$ Crucially, its incidence numbers are disaggregated by sex. Given that we study US patents, we use incidence estimates for the US.

To build the link between MeSH and GBD we had a research assistant go through the thousands of MeSH terms on the "Disease[C]" branch of the ontology and, when possible, match each MeSH term to the appropriate GBD disease topic. Where the research assistant was unsure, they marked them as such, and we adjudicated the decision. S14 reports our mapping from MeSH terms to GBD disease topics. The result is that, for each disease MeSH term, we know the GBD topic(s) it matches to and the annual estimated incidence by gender.

Using this crosswalk (Appendix S16), we construct a patent/GBD-topic dataset. Each row represents a GBD topic that our crosswalk says a patent addresses. We calculate the male and female incidence using the GBD estimates from the same year as the patent. ${ }^{4}$ The resulting dataset includes 662,727 patent/GBD-topic observations.

In Table S-8 we show that patents tagged as male/female are much more likely to address diseases that impact more men/women. In Model 1 we regress the logged female incidence count on whether the patent is tagged as female. We include the logged male incidence count to account for the possibility that female tagged patents might simply focus on diseases that afflict more women and more men. We find that female-tagged patents address diseases that impact $110 \%$ more women than patents without the tag. In Model 2 we also include a dummy for whether the patent is male focused. Doing so merely increases the magnitude on whether a patent is female tagged to $130 \%$. Models 3 and 4 are similar, but test the effect of being tagged as male on the logged male incidence. Though the effect sizes are smaller, we again find that male-tagged patents address diseases that impact $37 \%$ to $73 \%$ more men than women. The average female- or male-tagged patent is much more likely to benefit that sex.

\footnotetext{
${ }^{2}$ http://www.healthdata.org/gbd

${ }^{3}$ Here we analyze incidence, the total number of women with the condition, as against the incidence rate, which measures the share of women with a given condition.

${ }^{4}$ The GBD only goes back to 1990 . We therefore use the 1990 incidence rates for all pre-1990 patents.
} 


\section{S-3.4 Female/male tagged patents are more likely to be evaluated by female/male only medical trials}

Our fourth approach uses a patent's text to predict the probability that the invention described would be tested with a female-only or male-only clinical trial. Scientists and doctors regularly run trials on new interventions, drugs, or practices. As part of the trial design, they must decide on whether the trial recruits only female subjects, male subjects, or subjects of both sexes. On the one hand, these choices might still reflect erroneous gender and sex biases by doctors and scientists $(12,17)$. Indeed, before 1986 the NIH recommended that clinical trials exclude women. On the other hand, the NIH Revitalization Act of 1993 changed this rule and required the NIH to ensure that women and minorities were included in any relevant clinical trials. Recent years have seen a shift towards increasing diversity and representation amongst trial participants. This shift suggests that, at least for trials from the late 1990s onwards, the choice to include only men or only women in a trial reflects who doctors and scientists think will most benefit from the intervention, drug, or practice being tested.

To estimate the probability that an invention is more likely to be tested with a female-only or male-only trial, we rely on a semi-supervised transfer learning approach $(42,43)$. First, we take the full text from all the patents in our sample, along with the titles and abstracts from all publications from PubMed. Using this corpus of 6.6 billion words (comprising 3.72 million unique terms), we then estimate a 100-dimensional word representation using fastText. ${ }^{5}$ This approach, treating words as points in a high-dimensional vector space, has been successfully used and validated on similar biomedical data (44). We find that our simple word-embedding model reflects semantic space in a relatively rich way. For example, removing the word vector for "women" from "breast" and adding "men" yields a vector that is closest to the word "prostate," capturing the similarity of two types of cancer that especially impact women and men. While we hope that future work refines and improves on this approach, prior work has demonstrated that these word-embedding approaches are relatively robust, yielding broadly similar results even when fed different types and quantities of data or fit using different parameters (45).

We then use this word-embedding model in conjunction with data from clinicaltrials.

\footnotetext{
${ }^{5}$ We used fastText's (https://fasttext.cc) default model parameters, including the use of the simpler skipgram model to capture differences in word context.
} 
gov on 353,500 clinical trials that were started between 1994 and 2020. For each trial, we know whether it recruited men $(16,175)$, women $(34,656)$, or both sexes $(302,659)$. For each trial, we concatenate its title, summary text, list of conditions, and medical keywords into a single piece of text. These trials range from tests of "Surgery in Treating Patients with Prostate Cancer" (male only subjects) to "Intravenous Immunoglobulin Therapy in Optic Neuritis" (both sexes) to "Vaccine for Ovarian Cancer" (female only). While the female/male-only trials primarily focus on diseases that only impact women/men there are examples where the trials only enroll one sex even though the disease affects both. For example, a trial entitled "Impact of Sleep Disordered Breathing in Older Adults" decided to only enroll men, and the trial "Tailored Treatments of Fibromyalgia" decided to only enroll women, consistent with the fact that sleep disorders are more common in men and fibromyalgia is more common in women.

We then use fastText's supervised learning algorithm - a clever combination of the word representations and straightforward multinomial logistic regression - to train two models using this trial data, one that uses the trial text to predict whether the trial recruited only men and another that predicts whether the trial recruits only women. In both cases, the models are fed the 100dimensional word representation we built using the patent and PubMed data. While "transferring" these word representations does slightly improve model accuracy when predicting the enrollment sex of clinical trails, our primary aim is to improve the ability of our clinical-trial-trained models to predict whether the idea described in a patent, or PubMed abstract, is more or less likely to be evaluated with a female-only or male-only trial. By relying on the the Patent-PubMed word representation we implicitly map the clinical-trial text into the semantic space that reflects text from patents and PubMed abstracts, which in turn should improve the generalizability of these models for these texts.

To train and test our models, we randomly selected 340,000 trials as a training set and 13,500 clinical trails to serve as a test set. To be clear, this test set allows us to test the models precision/recall for clinical-trial data, but it does not tell us the accuracy when the algorithm is fed patent text. We find that that our models do exceptionally well at predicting the enrollment sex for clinical trials, with both models yielding precision and recall rates of $98 \%$ on the test set. Even when we account for label imbalance, and test our models on a sample that has an equivalent number of female/male and non-female/non-male trails, we find precision and recall rates of $86 \%$ 
for our male-only model and $94 \%$ for our female-only model. The textual content describing a clinical trial appears to be a strong predictor of whether the trial is likely to enroll only men or only women.

We then feed the first 2,500 characters of each patent's title, abstract, and summary - the same text we we fed to the MTI - through our two models. Table S-9 shows the estimated probabilities of a female-only trial for five randomly selected patents from each decile of the predicted female-onlytrial distribution. The table also lists the male-only-trial predicted probability, the patent's title, and a column indicating whether, after human review of the patent's text, the invention focuses on the medical needs of women. For example, the third patent mentions "feminine libido" in the title, and indeed reading the patent reveals that it is female focused. While the title of the top patent "Treatment of climacteric disorders with nitric oxide synthase substrates and/or donors" does not jump out as female focused, the text of the patent makes it clear that it is: the invention addresses conditions stemming from menopause, including hot flashes and female urogential discomfort. The patent "Connection mechanisms for uterine mobilizer" focuses on "gynecological" applications. The patent "Ultrasound device for use in a thermotherapy apparatus" is for treatment of breast cancer. Looking at Table S-9, it is clear that patents with higher estimated probabilities are, in fact, inventions that are more likely to be tested with women.

Similarly, Table S-10 shows the estimated probabilities for five randomly selected patents from each decile of the predicted male-only-trial distribution. Again, we find evidence that our algorithm trained with clinical trial data generalizes relatively well to our patent data. While the top patent's title "Certain phopohometyl..." does not jump out as male focused, the patent text makes clear that the invention address prostate cancer. The patent "Implantable penile prosthesis" is clearly male focused. Overall, both Tables S-9 and S-10 lend support to the idea that our trial-population sex estimates capture meaningful differences in who benefits from an invention.

Finally, in Table S-11 we regress our estimate for whether an invention is more likely to be tested with a female-only trial on the MTI-assigned sex tags. In Model 1 we find that the estimated femaletrial probability increases by 13.1 percentage points when the female MeSH tag is present. Given that the baseline probability is 4 percent, this effect reflects a $428 \%$ increase. In Model 2 we add in the male MeSH tag and find that the presence of the male tag reduces the probability of the invention's being tested with a female-only trial by 3.6 percentage points - essentially reducing that 
probability to zero. Model 3 dichotomizes our dependent variable at $80 \%$ to test if the check tag is applied to the inventions most likely to be tested with a female-only trial. We again find similar patterns, with the female MeSH tag increasing the probability by 9.9 percentage points from $0.21 \%$, a 48 -fold increase.

Table S-11 Columns 4-6 show similar models, but for our male-only trial estimates. Patents tagged as male are much more likely to be tested with male-only trials, with the relative effect in Column 6 reflecting a 21 -fold increase from a baseline of $0.23 \%$. That said, the association, though strong in and of itself, is weaker than the female estimates. There are a myriad of reasons why this might be, ranging from the idea that there may be more female-only biomedical conditions than male-only conditions to the notion that "male" is the default sex in medicine, which in turn makes it hard to separate male-focused inventions from "sex-neutral" ones.

Finally, it is worth noting differences and similarities in the MeSH and clinical-trial measures. While the MeSH tags suggest that just over $10 \%$ of patents are male/female focused, our trialbased measure is more conservative, suggesting that the rate is closer to a few percentage points. This difference is likely because this clinical-trial-based measure captures clear sex differences (e.g., trials for prostate cancer versus gestational diabetes), while our MeSH measure also picks up on more subtle differences (e.g., Parkinson's versus lupus). That said, both measures suggest that the sex-focus of inventions is split roughly evenly between the sexes. This is quite different than the trial data, where female-only trials outnumber male-only trials by two to one. We hope that future work explores this striking difference between the sex focus of inventions and of trials. 


\section{S-4 Inventor gender classification}

We used dictionary matching to assign gender to inventor names. We followed the dictionarymatching approach that Jensen et al. (26) describe in the supplement to their article. We differed slightly in the data sources for our dictionary, which we detail below. We assign gender only when the name in question maps to male or female at least 95 percent of the time it appeared in our dictionary. With this method, we could identify the gender of at least one inventor on $98 \%$ of patents, the lead inventor on $92 \%$ and the entire team on $80 \%$. This measure is necessarily a proxy, but one that follows practice in other innovation studies.

It is worth noting that after we began this project, PatentsView published a gender assignment for each inventor (http://patentsview.org/download). Our classification results correlate with PatentsView's at above 0.98, and using their measure does not change our findings.

It is also worth noting that our estimates rely on a binary definition of gender, though for many this dichotomization does not apply. We hope future work explores increasingly nuanced ways to measure an individual's gender. Such measures would allow us to better estimate biases and inequities faced by individuals from across the gender spectrum.

Table S-12 reports the proportion of patents for which we could assign gender to all of the inventors as function of the number of inventors listed on the patent, as well as for U.S.-based versus non-U.S.-based teams. One can readily see that our ability to identify the gender of all members of the team declines with team size. Our gender-identification algorithms also perform better among U.S.-based teams of inventors. As with many current algorithms, the majority of our non-matched records consist of Asian names.

We use the following sources for inventor gender assignment:

- United Kingdom Intellectual Property Organization (46)

Available https://www.gov.uk/government/publications/gender-profiles-in-worldwide-patentingan-analysis-of-female-inventorship

Description 102,777 unique names, of which 63,121 are female and 39,656 are male

- World Intellectual Property Organization (47)

Available http://www.wipo.int/publications/en/details.jsp?id=4125

Description 289,452 unique name-gender-nationality records, of which 157,066 are female, 112,207 are male, and 20,183 are undetermined. Combines records from the US Social 
Security Administration and Census Bureau, the Alberta government, the UK Office for National Statistics, Statistics Sweden, Spain's Instituto Nacional de Estatística, France's Institut National de la Statistique, and Denmark Statistics

- US Social Security Administration

Available https://www.ssa.gov/oact/babynames/limits.html

Description 1,924,665 names (97,310 unique), of which 1,138,293 are female (67,046 unique) and 786,372 are male $(40,927$ unique)

- Florida Voter Registration

Available https://dataverse.harvard.edu/dataset.xhtml?persistentId=doi:10.7910/DVN/UBIG3F

Description $13,710,231$ names (545,396 unique), of which $7,196,185$ are female $(368,313$ unique), 6,160,967 are male (194,150 unique), and 352,075 are undetermined $(56,567$ unique)

- New York City Baby Names

Available https://catalog.data.gov/dataset/most-popular-baby-names-by-sex-and-mothersethnic-group-new-york-city-8c742

Description 11,345 names (3,016 unique), of which 5,806 are female (1,629 unique) and 5,539 are male $(1,430$ unique)

- genderize.io

Available https://genderize.io

Description Gender information on 4,000 additional unique names. We kept the gender information only for names for which there were at least 20 samples in the database and that were assigned to one gender at least $95 \%$ of the time

- GenderAPI

Available https://gender-api.com

Description Gender information on 1,249 additional unique names. We kept the gender information only for names for which there were at least 20 samples in the database and that were assigned to one gender at least $95 \%$ of the time 


\section{S-5 Regression tables corresponding to Figure 4A}

Here we report the regression tables corresponding to Figure $4 \mathrm{~A}$ in the body of the paper along with the estimates that correspond to the analysis of heterogeneity presented in S9. Table S-13 shows the point estimates and standard errors from our baseline model. All models include fixed effects for the patent year interacted with the patent's subcategory and for the patent year interacted with the count of inventors listed on the patent (team size). We include the year $\times$ subcategory fixed effects to account for potentially time-varying differences in the gender focus of drugs, surgery, biotech, and other subcategories of inventions. This is especially important because the rise of female inventors has been concentrated in drug patents, where female-majority teams account for $25 \%$ of inventions in 2010, and has been stubbornly flat for Surgery and Instruments inventions, where the rate remains at $10 \%(\mathrm{~S} 9)$. Yet drug patents are much less likely to be sex-focused than Surgery and Instruments inventions. Thus, even if patents by women are more likely female-focused within both types of invention, these opposing likelihoods may dampen the magnitude of the underlying female inventor-invention link (i.e., Simpson's paradox). We include year $\times$ team-size fixed effects to account for the fact that larger teams mechanically have a higher chance of having at least one female inventor and because team sizes have been growing in the sciences (22).

Tables S-14, S-15, and S-16 show the estimates after exactly matching on a patent's year, subcategory, and narrow disease area. For example, we built column 1 in Table S-14 by first restricting the sample to those patents with either all-male or female-minority inventor teams. We then match each female-minority patent to a male patent from the same year and subcategory, with the same number of inventors and the same narrow disease area. The latter are level-four MeSH terms from the "Disease[C]" branch of the MeSH ontology and include terms like "Atrial Flutter [C14.280.067.248]," "Atrial Fibrillation [C14.280.067.198]," and "Pre-Eclampsia [C13.703.395.249]." By way of contrast, these narrow disease areas are nested within broader levelone, -two, and -three terms like "Neoplasms [C04]," "Heart Diseases [C14.280]," and "Hypertension, Pregnancy-Induced [C13.703.395]." Our findings also hold when we match on these broader MeSH terms. After matching the data, we then fit a model that includes disease $\times$ year $\times$ subcategory $\times$ teamsize fixed effects. We weight observations with the matching weights. Columns 2-8 in Table S-14 then report models with the same structure but that first restrict the data to the indicated sub- 
sample before matching. Tables S-15 and S-16 repeat the exercise for patents with majority-female invention teams and all-female invention teams. 


\section{S-6 Female inventors are neither more nor less likely to invent for men}

Figure S-4 replicates Figure 4A in the main paper and Figure S-5 discussed in S9, but predicts not whether a patent is female-focused, but whether it is male-focused. We find relatively well-estimated zeros across the different models and subsamples. It does not appear that female inventors are more likely to invent for both men and women. Tables S-17, S-18, S-19, and S-20 report the regression tables that correspond to the estimates in Figure S-4. See the preceding section for more details about these regressions and the matching process. 


\section{S-7 Robustness to alternative modeling assumptions, text pro- cessing, and dependent variables}

We conducted several robustness checks to test whether our results are robust to alternative modeling assumptions, data processing decisions, and alternative measures of a patent's female focus. The first two columns in Table S-21 report our baseline findings, which use a linear probability model. Column 1 includes the standard year, team-size, and subcategory fixed effects. Column 2 is similar but also adds in narrow disease-area fixed effects. ${ }^{6}$ The next two columns show that these results hold when we use logistic regression instead of OLS. Columns 5 and 6 show our results are largely unchanged if we classify patents as female-focused if (1) the patent is classified as femalefocused by the MTI and (2) is NOT classified as male-focused by the MTI. The final two columns show similar patterns when we use logistic regression and the female-only measure.

Table S-22 shows that our findings are robust to how much text we feed to and what options we select for the MTI algorithm. Column 1 shows estimates when we rely on the first 2,500 characters of the patent's title, abstract, and summary text (as we do in Figure 4A). Column 2 adds disease $\times$ year fixed effects. The models reported in columns 3 and 4 are similar but rely only on the title and abstract, again cutoff at 2,500 characters. The estimates are slightly smaller, but the pattern holds. Models 5 and 6 rely on MeSH terms produced using the MTI's "strict" filtering option and the first 2,500 characters from the title, abstract, and summary text. The strict option emphasizes precision by returning many fewer terms and using more complex word-sense-disambiguation algorithms. The disambiguation algorithms make the MTI much slower when using the strict options. As a result, it is infrequently used by researchers and by the National Library of Medicine. We again find similar, though somewhat smaller, results. Given that in S2 we find that the MTI algorithm has high precision for the "Male" and "Female" check tags, the strict filtering option may yield an overabundance of false negatives. Finally, columns 7 and 8 again use the regular filtering options but use MeSH terms produced from feeding the MTI 10,000 characters of a patent's title, abstract, and summary text. Our pattern of results holds; if anything, the effects are larger. This suggests

\footnotetext{
${ }^{6}$ We find similar results if we match and then estimate with fixed effects, instead of just including fixed effects. However, we largely rely on fixed effects in our robustness checks for the sake of simplicity of computation and reporting. See the next section for more detail on how the fixed-effects estimates compare to the models that first match and then include fixed effects.
} 
that trimming our analysis to the first 2,500 characters might lead us to understate the female inventor-invention link, especially for all-female invention teams.

Our findings are also robust if we limit our analysis to patents that are clearly focused on "humans" and not on inventions that might instead target other animals (e.g., improved cowmilking devices or castration devices for bulls). In Table S-23 Columns 1 and 2 we show that our results hold when we restrict our sample to all patents that are tagged by the MTI with the MeSH term "humans" - though this potentially excludes many pharmaceutical patents that target particular diseases but might not invoke human subjects or anatomy in great detail. Our results strengthen. In Columns 3 and 4 we show that our results hold if we exclude any patent that is tagged by the MTI with the MeSH term "animals." This would include breast cancer inventions describing prior work completed on mice. Again, our results are if anything slightly larger.

Our results also hold if we use alternative measures of a patent's sex focus. Model 1 in Table S-24 regresses our measure of female incidence for each disease a patent addresses on the gender composition of the inventor team (See Appendix S3 for further details on how we construct this measure). As with our the female MeSH tag, we find a clear dose-response relationship, with minority-female teams addressing diseases that impact $4 \%$ more women, majority-female teams addressing diseases that impact $7 \%$ more women, and all-female teams $16 \%$ more women. Model 4 is similar but focuses on log male incidence. We find that patents with all-female teams address diseases that impact $12 \%$ fewer men. Women are not merely doing more applied research, but appear to be especially likely to produce inventions that target diseases that afflict more women.

Model 2 in Table S-24 regresses our estimate of whether the patent describes an invention that would be tested with a female-only clinical trial on the gender composition of the inventor team (Again, See Appendix S3 for further details on how we construct this measure). We again see a dose-response relationship. Given that the average probability is just over 5\%, the effect for all-female teams, 2.8 percentage points, represents a healthy $50 \%$ increase. In column 3 we show that this result holds when we dichotomize our prediction of whether the invention will be tested with a female-only clinical trial at $80 \%$. We find similar results, with an estimated increase of 1.6 percentage points off a baseline of $1.1 \%$, a relative increase of nearly $150 \%$. Models 5 and 6 follow the structure of models 2 and 3 but test whether women invent for men. We find no such evidence; if anything, there is modest evidence that women are less likely to build inventions that would 
particularly benefit men.

Finally, we replicate the complete set of findings presented in Figure 4 of the paper and in Figure S-5 using our measure of whether an invention is more likely to be tested with a female-only clinical trial. Tables S-25, S-26, S-27, and S-28 present the regression results corresponding to our analysis of heterogeneity along with use of disease matching and fixed effects. We find an almost identical pattern of results, with the relative effect sizes largely similar. For example, after matching we find an estimate for all-female teams in Model 1 of S-28 of 1.6 percentage points, just over a $30 \%$ increase over the baseline rate, and still sizeable when compared to the $50 \%$ relative effect size we get without matching. The only notable difference is that, in Table S-25, the effect size is larger for corporate patents than for non-corporate patents. However, once we include diseaseyear-subcategory-team-size fixed effects the pattern reverses and, consistent with Figure S-5, we find that the effect size is larger in non-corporate settings.

Finally, it is worth noting that the standard errors we report change little if we cluster them on different combinations of patent year, team size, subcategory, and disease. 


\section{S-8 Accounting for selection with additional fixed effects and dou- ble selection lasso methods}

A key aspect of our analysis is that we show that female inventors are more likely to invent for women across and within narrow disease areas. In Figure $4 \mathrm{~A}$ we show that our findings hold when we match on the patent's year, subcategory, team size, and disease focus. Table S-29 shows that our findings hold when we instead match on fewer variables. Matching on disease-subcategory (odd-numbered columns) or disease-subcategory-year (even-numbered columns) yields essentially the same estimates. The only notable difference is that the coefficient for minority-female teams becomes somewhat larger and statistically significant.

While our exact-matching analysis is both stringent and simple (48), it does make two crucial simplifying assumption. First, we assume that patents are primarily associated with a single disease area. Second, we assume that we can model diseases as associated or not with with a patent. Instead, patents might regularly address multiple diseases. In this case, if we only control for the disease most associated with the patent, we might fail to capture the multi-dimensional nature of selection in terms of the areas where female inventors work. Further, perhaps there is meaningful variation in how strongly or weakly a patent is associated with a disease. If female inventors work on a more diffuse set of topics, and thus have patents that are more weakly associated with more diseases, then its possible our dichotomous measure linking patents to diseases might lead us astray.

We address the multiple-disease concern in Table S-30. We report fixed-effects models that include fixed effects for up to 10 different disease areas interacted with the year of the patent. The first fixed effect corresponds to the disease most associated with the patent according to the MTI. It is interacted with year to account for potential time-varying heterogeneity. The second fixed effect captures the disease next-most associated with the patent, again interacted with year. If a patent only matches to one disease then we set the value of the second fixed effect to "no matching disease." The most stringent model accounts for the ten diseases most associated with the patent. While including additional disease fixed effects weakens the effect for minority-female teams, if anything it appears to strengthen the effect for majority- and all-female teams.

We address the strength-of-association concern by relying on the fact that the MTI produces a confidence score for each MeSH term it assigns to a patent. Presumably, when a disease is more 
central to the patent, this score will be higher. This corresponds to the disease's being mentioned more, its different variants and related problems being described, its being mentioned in the title or abstract, and so on. To use this score, we first create a separate variable for each of the thousands of diseases in the MeSH tree that a patent could potentially match to. These variables are set to 0 when the MTI does not assign the disease to the patent, and take the logged confidence score when it does. ${ }^{7}$

Unfortunately, standard fixed effects and matching methods are ill-equipped to handle thousands of relatively, sparse continuous predictors. Instead, we turn to double selection lasso methods (49). This approach uses the lasso to select both the variables that drive selection (e.g., the disease areas where we see lots of both female inventors and inventions) and to select variables that serve as useful controls to improve power (e.g., diseases where the baseline rate of female focus is high or low irrespective of inventor composition). Furthermore, the double selection lasso reduces concerns that including so many controls leads to over-fitting, avoids "over-penalizing" controls as compared to a standard lasso model, and lets us fit models where the number of regressors is potentially bigger than the number of observations. At a conceptual level, the method has similarities to propensity score methods, but instead of including the estimated propensity score as a regressor to account for selection, you instead directly include the variables that the lasso algorithm identifies as driving selection.

Table S-31 shows results from models fit using the double selection lasso method. To account for potential time-varying heterogeneity in disease areas and to address the computational constraints of this method, we fit models to each decade of our data separately, lumping the late 1970s together with the 1980s. The models include our standard team-size, subcategory, and year fixed effects. We include our three covariates of interest - whether the team is minority-, majority-, or all-femaleand more than 4,000 disease-area predictors that the lasso then selects from. The pattern of results is similar to what we find when using fixed effects and matching. It appears that controlling for the disease most associated with a patent is largely sufficient to account for men's and women's sorting into different areas of invention within the biomedical sciences.

\footnotetext{
${ }^{7}$ We $\log$ scores since the distribution is extremely fat-tailed, with many scores close to 0 and some in the tens of thousands.
} 


\section{S-9 Heterogeneity in the female-inventor invention link}

Here we replicate Figure $4 \mathrm{~A}$ in the body of the paper but include additional panels to explore heterogeneity in the female inventor-invention link. Figure S-5 Panels B, C, and D split the sample by decades, to test whether the increase in women's representation muted the female inventorinvention link (The tables corresponding to Figure S-5 are presented in S5). As women entered more diverse research areas, they may have confronted potentially fewer undiscovered female-focused ideas. While the point estimates shrink by roughly a third between the 1970s-80s (Panel B) and the 2000s (Panel D), none of the differences are statistically significant. Instead the biggest shift is a pronounced dip in the 1990s (Panel C), perhaps related to the aforementioned push by the NIH to focus on women's health. As of 2010, women were still identifying more new opportunities to invent for women.

The next pair of estimates cut the data by whether the patent is assigned to a corporation or to a non-corporate entity like a university, individual inventor, or the government. Prior work finds that management directs and influences what researchers in corporate labs invent (50). Given the limited representation of women in management, it is likely that female inventors in corporations have less latitude to pursue female-focused opportunities. While we find noisy estimates for minority- and majority-female teams, we do find in panels $\mathrm{E}$ and $\mathrm{F}$ that all-female teams are 2.4 percentage points more likely to invent for women in non-corporate than in corporate settings across the unmatched and matched estimates $(p<0.001 ; p=0.063)$.

The final pair of estimates split the data by whether the patents are from the drug (55\% of patents) or surgery (35\% of patents) subclasses. While pharmaceutical research has seen an influx of female inventors, from $10 \%$ majority-female teams in 1976 to $25 \%$ by 2010, surgery has remained stubbornly male-dominated, in 1976 just under 10\% of patents were from female-majority teams; in 2010 just over $10 \%$ were (S9). Figures S-6 and S-7 illustrate these trends along with percent of patents by female-majority and male-majority teams that are female/male-focused. Especially given the history of hostility towards women in surgery (25), female inventors might be especially likely to see opportunities for women, or men especially likely to dismiss them. Indeed, in panels $\mathrm{G}$ and $\mathrm{H}$ we see that all-female teams are 8.7 percentage points more likely to invent for women in the surgery subcategory, versus 2.3 percentage points in drugs $(p<0.001)$. Further, this difference 
remains after matching $(p=0.036)$. 


\section{S-10 Building the biomedical publication dataset}

Our data on biomedical publications come the from the National Library of Medicine's PubMed database. We use the 2019 version of the PubMed data. This data is commonly used in studies of the science of science $(36,51,52)$. We extract each article's publication year, authors, journal of publication, and associated MeSH terms published or forthcoming from 2002 through 2020. We focus only on original research articles and exclude literature reviews, books, and other non-journalarticle entries that are included in Pubmed but unlikely to ever be cited by or commercialized as a patent. While the PubMed data goes back before 2002, it only records each author's full first name (as opposed to the first initial) after 2001. Following the procedure described in S3, we use the the full first names to assign authors' genders.

As noted in S2, unlike patents, most articles in the PubMed dataset are assigned about ten MeSH terms by human indexers before being added to the database. After filtering out roughly three million articles where MeSH terms are absent ${ }^{8}$ and articles where we cannot assign author gender we end up with a sample of 5,529,360 research publications. Since our interest is in seeing if women who are at risk of inventing are more likely to discover female focused ideas we further restrict our sample to the $2,062,695$ articles published in the top 1,000 journals in terms of commercial impact factor (53). Articles published in these journals are the most likely to be cited in or serve as the basis of a patented invention. See Bikard and Marx (53) for further details on the construction of the journal commercial impact factor metric.

We again use the "Female" and "Male" check tags to measure an article's sex focus. Again, it is worth emphasizing that these check tags are added by human indexers and not by the MTI's algorithm. To measure a patent's disease area we again use the level-4 disease MeSH terms, as we do in our patent analysis. In the minority of cases where a patent matches to multiple levelfour MeSH disease terms, we randomly selected one to use in our analysis. ${ }^{9}$ See S14 for summary statistics.

\footnotetext{
${ }^{8}$ These non-indexed articles are generally non-biomedical articles about health, for example health-economics papers, that are also included in PubMed.

${ }^{9}$ Unlike the MeSH terms produced by the MTI algorithm, human-assigned MeSH terms are not ranked by the human indexer, so we cannot use a score to choose. That said, the human indexers rarely assign more than ten MeSH terms, whereas the MTI assigns dozens. As a result, articles overwhelmingly match to only a single specific disease.
} 


\section{S-11 Regression table corresponding to Figure 4B}

Table S-32 displays the point estimates and standard errors for the models reported in Figure 4B of the paper. Column 1 reports an OLS regression of an article's female focus on the gender composition of the research team. It includes year $\times$ team-size and year $\times$ journal fixed effects. Columns 2 through 4 present estimates after exactly matching on disease $\times$ year $\times$ team size and includes fixed effects for the matching variables along with year $\times$ team-size and year $\times$ journal fixed effects. To match, we take papers with minority-, majority-, and all-female teams and then exactly match them to papers all male teams with the same number of authors, published in the same year, and addressing the same disease area. 


\section{S-12 Publication analysis robustness checks}

Here we report a number of robustness checks. First, we validate that publications tagged as female or male tend to address women's or men's health. Second, we show that female inventors are not more likely to discover for men. Third, we show our results hold if we analyze articles that are more or less "commercializable," hold under alternative matching schemes, and hold when we analyze shifts overtime or in terms of authorship order. Fourth, we show that our findings hold when we use alternative measures for whether a research article focuses on the needs of women or men. Fifth and finally, we connect our publication and patent analysis to show that patents by women are also more likely to build on female-focused research.

\section{S-12.1 Do female/male tagged publications address the health needs of women/men?}

Here we follow the analysis in Appendix S3 to show that female/male tagged biomedical publication are more likely to focus on female/male health needs. Validating that the female/male MeSH tags capture differences in the sex focus of publications is especially important because prior research has relied on these sex tags to measure whether a research article reports the sex of the subjects studied or analyzes heterogeneous effects by sex $(39,54)$. However, a publication's sex focus and sex reporting are likely correlated. As (39) note, single-sex studies - such as a research article describing a new diagnostic method for ovarian cancer-de facto engage in sex reporting, since the topic clearly indicates subject sex. Thus, while prior work suggests that the female/male MeSH tag reflects whether a patent reports information about sex, it remains unknown whether female/male tagged articles are also meaningfully more focused on the medical needs of women/men.

Tables S-33 and S-34 show the words most associated with the articles tagged as female/male by PubMed indexers. We constructed the list following the weighted logs-odds approach described in S3 and only display terms that occur at least 100 times. We find strong evidence that the words differ in ways that suggest female/male patents are female/male focused. For example, in Table S-33 the top word is cin3, an abbreviation for "Cervical intraepithelial neoplasia," a form of cervical cancer. The other words include endometriosis, transvaginal, fallopian, and mothers. The same is true for male-tagged patents. The top term in Table S-34 is "iief," an abbreviation for "International Index of Erectile Function." The other words include testicular, ejaculation, male, 
and psma (prostate-specific membrane antigen). Intriguingly, there are a number of terms related to heart conditions that disproportionately impact men.

In Table S-35 we show that female/male MeSH-tagged publications target diseases that impact more women/men. Using data on disease incidence by sex, described in S3, we map each publication to our incidence data using the disease MeSH tags applied to each publication. To simplify our analysis, we use incidence estimates from the US from 2010. In Column 1 we show that that patents tagged as female address diseases that impact $200 \%$ more women than those not tagged as female. This is not the result of female-tagged patents merely addressing higher incidence diseases, because we control for the logged male incidence rate. Column 2 controls for the male MeSH tag. Our results hold; the male MeSH tag predicts that the publication will address diseases that impact fewer women. Models 3 and 4 replicate the first two columns but predict log male incidence. Again, we find that the male MeSH tag predicts that the publication addresses diseases that particularly impact men.

Our final validation exercise uses the algorithm we developed in S-3 to predict the probability that the idea in a publication would be tested with a female- or male-only clinical trial. Tables S-36 and S-37 show that the algorithm appears to generalize well to publications, especially for female focused conditions, correctly estimating that "Vaginal mesh extrusion associated with use of ..." would likely be tested only on women and "Magnetic resonance imaging is a complementary method to stereological measurement of testicular volume" would be tested only on men. We use this algorithm to estimate these probabilities for all publications in our data. In Table S-38 we show that publications tagged as female/male are significantly more likely to describe ideas that would likely be tested with a female/male-only clinical trial. Indeed, Column 3 suggests that publications tagged with the female check tag are an order of magnitude more likely to have an estimated probability of a female-only clinical trail that is greater than $80 \%$.

\section{S-12.2 Are female scientists more likely to discover for men?}

Table S-39 replicates Figure 4B in the paper (Table S-32 in this appendix) but uses whether the publication is tagged with the male MeSH tag as the dependent variable. We find little evidence that papers by women are more likely to target the needs of men. 


\section{S-12.3 Our results are robust to alternative commercialization potential sample cuts, matching approaches, authorship ordering, and time period sub- samples}

Table S-45 shows that our results hold when we include publications with more or less commercialization potential. As discussed in S-10, we restrict our primary analysis sample to the top top 1,000 journals in terms of commercial impact factor (JCIF) (53). Columns 1 and 2 in Table S-45 show that our findings hold when we analyze all five million publications regardless of the journal's JCIF. The first column includes our baseline fixed effects, the second column fixed effects for disease $\times$ year. Columns 3 and 4 show the estimates using our sample of articles published in the top 1,000 JCIF journals. Columns 5 and 6 show the results when we look at articles in the top 500 JCIF journals, Columns 7 and 8 the top 100.

Table S-41 shows that our findings in Figure 4B of the paper (Table S-32 in this appendix) are robust to different matching approaches. Unfortunately, exactly matching on year (18 years), team size (dozens), disease area (of which there are thousands), and journal $(1,000)$ quickly leads to a computationally infeasible 400 million potential combinations. However, we can show that our results hold when we match on the journal (Columns 1-3), instead of the disease area, even when we further include disease $\times$ year fixed effects (Columns 4-6).

Table S-42 explores heterogeneity in our effects, both by time period and by authorship order. For simplicity, we include fixed effects by year for the journal, team size, and disease area in all our models. In Column 1 we test if authorship order matters. We find some evidence that, at least for female-majority teams, articles with female first/last authors are more likely to be female focused. Columns 2 and 3 split this analysis by time period, 2002-2012 and 2013-2020. We find no significant changes across time.

\section{S-12.4 Our publication-analysis results hold when we use alternative female focus measures}

Our results are also hold if we use alternative measures of a publication's sex focus. See S3 for details on these measures. Model 1 in Table S-43 regresses our measure of female incidence for each disease a publication addresses on the gender composition of the authors. Majority- and all-female 
teams address diseases that impact roughly $45 \%$ more women. Model 4 shows that female scientists are less likely to address diseases that disproportionately impact men.

Model 2 in Table S-43 regresses our estimate of whether the research article describes an idea that would be tested with a female-only clinical trial on the gender composition of authors. We see a clean dose-response relationship. Given that the average probability is just $6.7 \%$, the effect for all-female teams, 2.6 percentage points, represents a healthy $39 \%$ increase. In column 3 we show that this result holds when we dichotomize our prediction of whether the idea in the paper will be tested with a female-only clinical trial at $80 \%$. If anything we find stronger results. Models 5 and 6 follow the structure of Models 2 and 3 but test whether women invent for men. We find no such evidence; if anything there is modest evidence that women are less likely to discover ideas that would particularly benefit men.

\section{S-12.5 Patents by women are more likely to build on female focused research}

In our final set of publication robustness checks, we test whether patents by women are also more likely to commercialize these female-focused research ideas. If they are, this provides further evidence that increasing the number of female inventors will lead to more female-focused science being incorporated into new inventions and thus more female-focused innovations. Unlike our patent or publication analysis, testing this hypothesis requires (1) mapping patents to the underlying research idea they commercialize and (2) measuring whether the research ideas that the patent commercializes are female-focused. We do so in two ways. First, we look at patent-paper "pairs," that is, patents where the inventor appears to be commercializing a scientific ideas that they themselves have discovered. By using the the sex focus of the "paired" articles, we can determine whether the patent is commercializing more female-focused ideas. We identify patent-paper pairs using patent-to-paper citations from Marx and Fuegi (55).

To do this, we restrict our attention to citations to articles that appear in the the PubMed database. As described in S9, MeSH terms are applied to articles in PubMed, so we can measure an article's sex focus using the "Female" check tag. To get at cited papers that are "pairs," we also restrict the citations to articles where the full name of at at least one inventor appears in the cited article. Finally, we restrict the cited articles to those added by the applicant (not by the examiner) that appear on the patent's front page, to ensure that the invention fundamentally depends on the 
citations. $^{10}$

The result is a dataset with 22,666 patents that on average match to three inventor-produced scientific articles. We classify a patent as drawing on female-focused knowledge if the majority of the cited articles are classified as female-focused in PubMed. Models 1 and 2 in Table S-45 show that patents with female inventors who are commercializing their own teams' research are more likely to be commercializing female-focused work.

The second way we measure knowledge inputs is by linking patents to NIH grants. (See (52) for further details and references on the NIH grant system.) The NIH requires researchers to disclose any publications or patents that come out of NIH-grant-funded research. Using the NIH ExPORTER tool, ${ }^{11}$ we link patents to NIH grants and grants to articles in the PubMed database. Since NIH grants can run for many years and can support research and patents that take decades to produce, we need to be careful to ensure the research is something the patent could plausibly be commercializing. We do so by restricting our analysis to articles associated with the NIH grant but that were published at most 5 years before the patent.

Our final dataset includes 12,834 patents. Each of these patents is linked to an NIH grant that had one or more publications in the five years before the patent. We classify the patent as commercializing female-focused research if $50 \%$ or more of the articles coming out of the grant are female-focused. Models 3 and 4 in Table S-45 show that patents by female inventors are substantially more likely to commercialize female-focused NIH-funded research.

\footnotetext{
${ }^{10}$ We also restrict the citations to those with a confidence score of 10 . See Marx and Fuegi (55) for further details.

${ }^{11}$ https://exporter.nih.gov
} 


\section{S-13 Back-of-the-envelope calculations of lost female-focused patents}

Here we estimate the potential number of lost female-focused patents and ideas that stem from the under-representation of women in invention and discovery. To do so, we assume that the number of patents and the female-focus rate by inventor gender remain constant. We then estimate how many more patents would be female-focused if women and men were equally represented as inventors. While this gives us a crude estimate for the number of lost female-focused patents, it ignores many factors that might move the estimate up or down. As we discuss in the paper, women might displace men from working on female-focused ideas, leading our estimate to be too high. Alternatively, increasing the number of female inventors might lead to an improved allocation of talent, in which there would be both more female-focused inventions and more patents overall. In this case, our estimate would be too low. We hope future work can explore these mechanisms. None of our findings speak to potential changes in patent quality when the inventor gender mix shifts. All that said, understand the potential magnitudes is still useful.

Our first estimate builds on Figures 1 and 2 in the body of the paper and calculates the number of lost female-focused patents by year. For each year, we assume the number of patents stays constant and that the female-focus rate for female-majority and male-majority teams stays constant. We then estimate the number of female-focused patents if $50 \%$ of patents came from female-majority teams. The number of lost female-focused patents, pictured in Figure S- 8 , is simply the difference between this estimate and the actual count. Indeed, in all years we find that there are potentially lost female-focused inventions. Overall, this approach yields an estimate of 3,168 lost female-focused patents. However, it fails to take into account that all-female teams are particularly likely to invent for women, which might bias our estimate downwards.

Our second estimate takes into account the fact that all-female teams are especially likely to invent for women, by using the between and within regression in Figure $4 \mathrm{~A}$ to estimate the number of lost female-focused inventions. Building off the fact that the mean invention team has 3 inventors in our patent data, we note that, if women were equally represented, then the average team would have $12.5 \%$ of patents from all-male, $12.5 \%$ from all-female, $37.5 \%$ from majority-female, and $37.5 \%$

from minority-female teams. In the data, we find that $72 \%$ of teams are all-male while $15 \%$ are minority-female, $9 \%$ are majority-female, and only $4 \%$ are all-female (See S12). Using the estimates 
from Model 1 in Table S-13 we then calculate the expected number of patents using the observed gender composition of invention teams and the counterfactual version where women are equally represented. We find that equalizing the number of women would have resulted in 6,534 more female-focused patents from 1976 through 2010.

That said, our estimate above fails to account for the sorting of women into more female-focused areas of invention. To account for this, we run the same exercise as above but use our within area estimates from Figure 4A which take into account differences in the disease areas that women work in. Doing so yields an estimate of 3,668 lost patents. This represents roughly $1 \%$ of all biomedical patents, an effect size that we think is both meaningful and plausible. It represents an effect size of just under 10\%, compared to the just over 50,000 female-focused patents we observe in in the data. Further, shifting the direction of existing researchers is non-trivial. (51) finds that it requires quadrupling the amount of funding a scientist receives to get them to to shift their research topics by one standard deviation. Any less, and the scientist will continue researching what they already focus on. How large is a one-standard deviation shift in direction? It is the difference between "a virologist deciding which of two closely related viruses to study for their next project." Our findings suggest that increasing inventor diversity, beyond simply being socially just, may also be an effective way to shift the direction of invention towards the needs of women.

Finally, we complete the same exercise using PubMed scientific articles to get a sense of whether the the researcher gender gap has potentially led to a meaningful loss of female-focused "ideas." Given that the average article from 2002 to 2020 in PubMed has five authors, and again assuming that women and men are equally represented as researchers, we end up with counterfactual gender breakdowns of $3.125 \%$ for all-male and -female teams and $48.4375 \%$ for majority- and minorityfemale teams. The observed rates are $34 \%$ for all-male teams, $38 \%$ for minority-female teams, $23 \%$ for majority-female teams, and $5 \%$ for all-female teams. This final number is likely the result from homophily among female researchers. Even so, the majority- and minority-female rates are still much lower than what we would expect if the inventor population were evenly split and teams were not formed on the basis of gender.

Using the estimates from Figure 4B, we find that there are potentially 39,658 lost female-focused discoveries amongst articles that could be published in the top 1,000 JCIF biomedical journals due to the research gender gap; 36,450 after accounting sorting by journal and disease area. While 
much more research is needed, there appears to be a substantial number of lost inventions and ideas that could benefit women. 


\section{S-14 Summary statistics}

Here we report summary statistics for the patent and PubMed data.

Table S-46 shows cross-tabs for the number of female-focused and male-focused inventions by the gender composition of the inventor team.

Table S-47 provides summary statistics for the sample we use in Figures 1 and 2. Table S-48 provides summary statistics for the sample we use in Figure $4 \mathrm{~B}$ of the paper.

We also provide summary statistics for our patent and scientific-article data for the years 2002 through 2010 in Tables S-49 and S-50. This allows a direct comparison on the sex-focus and gendercomposition differences over the same time period. Compared to our patent data, there are both more female-focused articles and more women researchers. 


\section{S-15 Four example patents along with MTI-assigned MeSH terms}

We include four patents here to give the reader a sense of the patent text and why patents are classified as male- or female-focused. We include a patent that matched to neither the male nor the female MeSH terms, a patent that matched only to the female term, a patent that matched only to the male term, and a patent that matched to both. We present the first page of these four patents below along, with matching MeSH terms, match rank, and MTI scores.

- Neither male nor female: Retractable needle syringe

- Male-focused: Gene expression markers for colorectal cancer prognosis

- Female-focused: Methods of diagnosing endometriosis

- Male- and female-focused: Combinations of vasoactive agents and their use in the treatment of sexual dysfunction 


\section{United States Patent}

Pace et al.

$[5$
$[7$
$[5]$
$[5$
$[5$

[54] RETRACTABLE NEEDLE SYRINGE

[76] Inventors: Paul A. Pace. 610 67th St.. 2nd Floor Front. Brooklyn. N.Y. 11220; John J. Riddle. 7804 3rd Ave.. Brooklyn. N.Y. 11209

[21] Appl. No.: 800,956

[22] Filed: Feb. 18, 1997

[51] Int. $\mathrm{Cl}^{6}$

[52] U.S. Cl. ...

..........................

97

A61M 5/00 604/195; 604/218 $604 / 187.604 / 195,192$

[56]

\section{References Cited \\ U.S. PATENT DOCUMENTS}

$4,919,657 \quad 4 / 1990$ Haber et al. ........................... 604/232

$4,950,251 \quad 8 / 1990$ Haining ……………................. 604/195

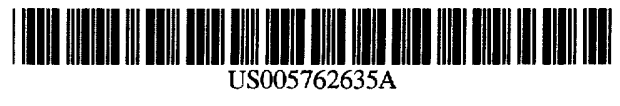

[11] Patent Number: $\quad \mathbf{5 , 7 6 2 , 6 3 5}$

[45] Date of Patent: Jun. 9, 1998

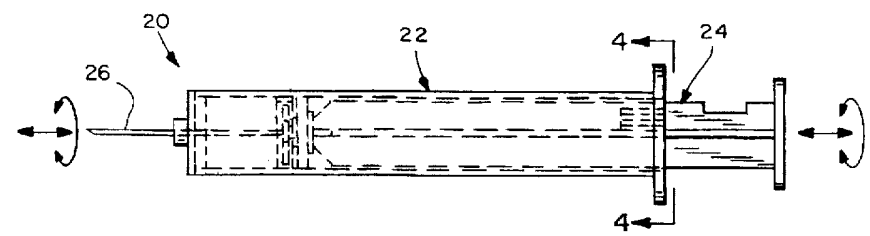


patent_mesh_tag_rank_count patent_mesh_score mesh_term mesh_tree_num

16023 HUMANS B01.050.150.900.649.313.988.400.112.400.400

25023 SYRINGES E07.877

32025 NEEDLES E07.612

4124 EQUIPMENT DESIGN E05.320

574 HEPATITIS A C02.440.420

667 MEDICAL WASTE DISPOSAL D20.944.460.300

763 HEALTH PERSONNEL M01.526.485

858 DRUG USERS M01.169

941 PLASTICS D25.720.716

1035 EQUIPMENT AND SUPPLIES E07

1135 INSTRUMENTATION

1232 PUNCTURES E02.800

1321 INVENTIONS J01.897.400

1415 SANITATION H02.229.782

159 SKIN A17.815

168 PHYSICIANS M01.526.485.810

177 OCCUPATIONS N01.824.547

186 WOUNDS AND INJURIES C26

196 CONTRACTS I01.880.604.583.090

205 HOUSEHOLD ARTICLES J01.494

214 DENTAL INSTRUMENTS E06.186.501

224 HOUSEHOLD PRODUCTS J01.516

232 COMMERCE J01.219

242 BIOLOGICAL TRANSPORT G03.143

252 POPULATION GROUPS M01.686

261 PHARMACEUTICAL PREPARATIONS D26

271 CREATIVITY F01.752.264

281 LIFE K01.752.400

291 COSTS AND COST ANALYSIS N03.219.151 


\section{S-15.2 Example male-focused patent: Gene expression markers for colorectal cancer prognosis US8367345}

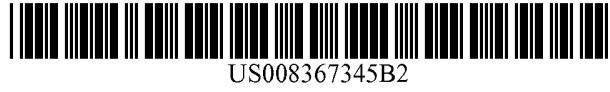

(12) United States Patent

\section{Cowens et al.}

(54) GENE EXPRESSION MARKERS FOR COLORECTAL CANCER PROGNOSIS

(75) Inventors: Wayne Cowens, Tiburon, CA (US); Joffre B. Baker, Montara, CA (US); Kim Clark-Langane, Sunnyvale, $\mathrm{CA}$ (US); James Hackett, San Jose, CA

(US); Drew Watson, Los Altos, CA (US); Soonmyung Paik, Pittsburgh, PA (US)

(73) Assignees: Genomic Health Inc., Redwood City, CA (US); NSABP Foundation, Inc. Pittsburgh, PA (US)

(*) Notice: Subject to any disclaimer, the term of this patent is extended or adjusted under 35 U.S.C. 154(b) by 0 days.

(21) Appl. No.: 13/413,338

(22) Filed: Mar. 6, 2012

(65)

Prior Publication Data

US 2012/0171688 A1 Jul. 5, 2012

\section{Related U.S. Application Data}

(62) Division of application No. 13/009,733, filed on Jan. 19,2011 , now Pat. No. $8,198,024$, which is a division of application No. 12/696,934, filed on Jan. 29, 2010, now Pat. No. 8,153,378, which is a division of application No. 11/653,102, filed on Jan 11, 2007, now Pat. No. 7,695,913.

(60) Provisional application No. 60/758,392, filed on Jan. 11,2006 , provisional application No. 60/800,277, filed on May 12, 2006, provisional application No. 60/810,077, filed on May 31, 2006.

(51) Int. Cl.

C12Q 1/68 (2006.01)

(52) U.S. Cl. ................................ 435/6.14; 435/6.12

(58) Field of Classification Search ....................... None See application file for complete search history.

(56)

\section{References Cited}

U.S. PATENT DOCUMENTS

$6,692,916$ B2 2/2004 Bevilacqua et at.

$6,960,439$ B2 $\quad 11 / 2005$ Bevilacqua et a

$6,964,850$ B2 $11 / 2005$ Bevilacqua et al.

$7,695,913$ B2 $4 / 2010$ Cowens et al.

$7,767,391 \quad$ B2 $\quad 8 / 2010$ Scott et al.

$8,026,060$ B2 9/2011 Watson et al

$8,029,995$ B2 10/2011 Watson et al.

$2001 / 0044414$ A1 11/2001 Clark et al.

2002/0150922 A1 10/2002 Stolk et al

$2002 / 0172987$ Al 11/2002 Terstappen et al.

2003/0077568 Al 4/2003 Gish et al.

2003/0109690 Al 6/2003 Ruben et al.

2003/0148314 A1 8/2003 Berger et al.

2003/0148410 Al 8/2003 Berger et al

2003/0166064 Al 9/2003 King et al.

2003/0198970 Al 10/2003 Roberts

2003/0219760 Al 11/2003 Gordon et al.

$2003 / 0225526$ Al 12/2003 Golub et al.

2004/0053317 Al 3/2004 Glinskil

$2005 / 0014165$ Al $1 / 2005$ Lee et al.

$2006 / 0195269$ A1 8/2006 Yeatman et a

2006/0211036 Al 9/2006 Chou et al.
(10) Patent No.: $\quad$ US 8,367,345 B2

(45) Date of Patent: $\quad$ Feb. 5, 2013

2007/0166704 A1 7/2007 Huang et al.

2009/0258795 A1 10/2009 Cowens et a

2009/0305277 A1 12/2009 Baker et al.

2010/0285980 A1 11/2010 Shak et al.

2010/0291573 A1 11/2010 Cowens et al.

2011/0039269 Al 2/2011 Cowens et al.

2011/0039271 A1 2/2011 Cowens et al.

2011/0097759 A1 4/2011 Cowens et al.

\begin{tabular}{lrr} 
& \multicolumn{2}{r}{ FOREIGN PATENT DOCUMENTS } \\
EP & $1522594 \mathrm{~A} 2$ & $4 / 2005$ \\
EP & $1274865 \mathrm{~B} 1$ & $2 / 2007$ \\
WO & WO9964626 A2 & $12 / 1999$ \\
WO & WO9964627 A2 & $12 / 1999$ \\
WO & WO0024940 A1 & $5 / 2000$ \\
WO & WO0141815 A2 & $6 / 2001$ \\
WO & WO0212280 A2 & $2 / 2002$ \\
WO & WO0212328 A2 & $2 / 2002$ \\
WO & WO0224956 A2 & $3 / 2002$ \\
WO & WO03050243 A2 & $6 / 2003$ \\
WO & WO03062395 A2 & $7 / 2003$ \\
WO & WO2004110345 A2 & $12 / 2004$ \\
WO & WO2005000087 A2 & $1 / 2005$ \\
WO & WO2005015236 A2 & $2 / 2005$ \\
WO & WO2005076005 A2 & $8 / 2005$ \\
WO & WO2005100593 A1 & $10 / 2005$ \\
WO & WO2006010150 A2 & $1 / 2006$ \\
WO & WO2006081248 A2 & $8 / 2006$ \\
WO & WO2006110581 A2 & $10 / 2006$ \\
WO & WO2007061876 A2 & $5 / 2007$ \\
WO & WO2007070621 A2 & $6 / 2007$ \\
WO & WO2007073220 A1 & $6 / 2007$ \\
WO & WO2007112330 A2 & $10 / 2007$
\end{tabular}

OTHER PUBLICATIONS

Anjomshoaa A., et al., "Reduced Expression of a Gene Proliferation Signature is Associated with Enhanced Malignancy in Colon Cancer," British Journal of Cancer, 2008, vol. 99, pp. 966-973.

Arango et al., "Gene-Expression Profiling Predicts Recurrence in Dukes' C Colorectal Cancer", Gastroenterology, 2005, vol. 129, No. 3, pp. 874-884

Augenlicht et al., "Low-Level C-Myc Amplification in Human Colonic Carcinoma Cell Lines and Tumors: A Frequent, p53-Independent Mutation Associated with limproved Outcome in a Randomized Multi-Institutional Trial," Cancer Research, 1997, vol. 57, pp. $1769-1775$.

Avvisato et al., "Mechanical Force Modulates Global Gene Expression and $\beta$-catenin Signaling in Colon Cancer Cells," Journal of Cell Science, 2007 , vol. 120 , pp. 2672-2682.

(Continued)

Primary Examiner - James Martinell

(74) Attorney, Agent, or Firm - Paula A. Borden; Bozicevic, Field \& Francis LLP.

\section{ABSTRACT}

A method of predicting clinical outcome in a subject diagnosed with colorectal cancer comprising determining evidence of the expression of one or more predictive RNA transcripts or their expression products in a biological sample of cancer cells obtained from the subject.

20 Claims, 1 Drawing Sheet 
patent_mesh_tag_rank_count patent_mesh_score mesh_term mesh_tree_num

161210 AGED M01.060.116.100

261210 HUMANS B01.050.150.900.649.313.988.400.112.400.400

361210 MIDDLE AGED M01.060.116.630

461210 MALE

560210 COLORECTAL NEOPLASMS C06.405.249.411.307

650166 PROGNOSIS E01.789

734404 COLONIC NEOPLASMS C06.301.371.411.307.180

87053 NEOPLASM STAGING E01.789.625

92600 ADENOCARCINOMA C04.557.470.200.025

102273 RECTAL NEOPLASMS C04.588.274.476.411.307.790

111111 GENETICS H01.158.273.343

121000 NEOPLASM RECURRENCE, LOCAL C23.550.727.655

13897 LYMPH NODES A15.382.520.604.412

14663 GENETIC MARKERS D23.101.387

15391 COLON A03.556.124.526.356

16145 THERAPY

17102 RECTUM A03.556.124.526.767

1873 DISEASE PROGRESSION C23.550.291.656

1933 SURGERY

2013 UNITED STATES Z01.107.567.875

2111 DIAGNOSIS E01

2211 DEATH C23.550.260

239 RNA D13.444.735

248 DIAGNOSTIC TESTS, ROUTINE E01.370.395

257 ETIOLOGY

265 EUROPEAN UNION I01.615.500.475

275 CLASSIFICATION L01.100

284 INVENTIONS J01.897.400

294 NEW YORK Z01.107.567.875.075.437

303 METHODS E05.581

312 DECISION MAKING F02.463.785.373

321 TIME G01.910 


\section{S-15.3 Example female-focused patent: Methods of diagnosing endometriosis US8247174}

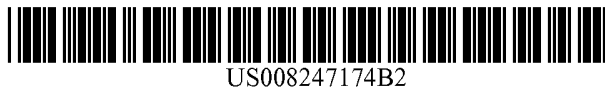

\section{(12) United States Patent Giudice}

(54) METHODS OF DLAGNOSING ENDOMETRIOSIS

(75) Inventor: Linda C. Giudice, Los Altos Hills, CA (US)

(73) Assignee: The Regents of the University of California, Oakland, CA (US)

(*) Notice: Subject to any disclaimer, the term of this patent is extended or adjusted under 35 U.S.C. 154(b) by 0 days.

(21) Appl. No.: 12/970,576

(22) Filed: Dec. 16, 2010

\section{Prior Publication Data}

\section{Related U.S. Application Data}

(63) Continuation of application No. 12/109,099, filed on Apr. 24, 2008, now Pat. No. 7,871,778.

(60) Provisional application No. 60/914,018, filed on Apr $25,2007$.

(51) Int. Cl C12Q 1/68 (2006.01)

(52) U.S. Cl.

(58) Field of Classification Search $435 / 6.1$ ............. None See application file for complete search history.

(56)

\section{References Cited}

U.S. PATENT DOCUMENTS

2003/0077589 A1* 4/2003 Hess-Stumpp et al. .......... 435/6

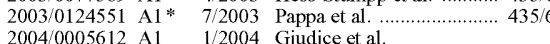

OTHER PUBLICATIONS

Talbi et al. (Endocrinology. Mar. 2006;147(3): 1097-121. Epub Nov. $23,2005) * *$

Attia, Georgia, et al. "Progesterone Receptor Isoform A but not B is Attia, Georgia, et al. "Progesterone Receptor Isoform A but not B is \& Metabolism, vol. 85, No. 8, pp. 2897-28902, Year: 2000.

Bulun. SE, et al. "Progesterone resistance in endometriosis: link of Bulun, SE, et al. "Progesterone resistance in endometriosis: link of
failure to metabolize estradiol," Mol. Cell. Endocrinol., 2006, vol. 248 , No. 1-2, pp. 94-103

Burney, Richard O. et al., "Gene Expression Profiling of Eutopic Endometrium from Women with Moderate-Severe Endometriosis," presented at the SRM meeting Oct. 21-25, 2006.

Burney, Richard O. et al., "Gene Expression Analysis of Endometrium Reveals Progesterone Resistance and Candidate Susceptibility Genes in Women with Endometriosis," Endocrinolog (Aug. 2007), 148(8):3814-3826.

Burney, Richard O et al ."Molecular Profiling Reveals Progesterone Burney, Richard O. et al., "Molecular Profiling Reveals Progesterone

(10) Patent No.: $\quad$ US 8,247,174 B2 (45) Date of Patent: $\quad$ Aug. 21, 2012
Endometriosis," presented at the $2^{\text {nd }}$ SGI International Summit held Nov. 7, 2007

Cullinan, Emily, et al. "Leukemia inhibitory factor (LIF) and LIF receptor expression in human endometrium suggest a potential autocrine/ paracrine function in regulating embryo implantation," Proc. Natl. Acad. Sci., 1996, vol. 93, pp. 3115-3120.

Igarashi, TM., et al. "Reduced expression of progesterone receptor-B in the endometrium of women with endometriosis and in cocultures of endometrial cells exposed to 2,3,7,8-tetracholordibenzo-p-dioxin," Fertil Steril, 2005, vol. 84, No. 1 pp. 67-74.

Kamat, A., et al., "Protein expression profiling of endometriosis Validation of 2-mm tissue microarrays," Fertility and Sterility, 2004 , vol. 82, Issue 6, pp. 1681-1683.

Kao, L.C., et al. "Expression Profiling of Endometrium from Women Kao, L.C., et al. "Expression Profiling of Endometrium from Wome C. Disease Based Implantation Failure and Infertility," Endocrinology, 2008, vol. 144 No. 7, pp. 2870-2881.

Lessey, Bruce, et al. "Aberrant Integrin Expression in the Endometrium of Women with Endometriosis," Journal of Clinical Endocrinology and Metabolism, 2004, vol. 79, No. 2, pp. 643-649. Lessey, Bruce, et al. "Integrin Adhesion molecules in the Human Endometrium; Correlation with the Normal and Abnormal Menstrual Cycle," 1992, vol. 90, pp. 188-195.

Osteen, KG, et al. "Reduced progesterone action during endometrial maturation: a potential risk factor for the development of endometriosis," Fertil Steril, 2005, vol. 83, No. 3, pp. 529-537.

Talbi, S. et al., "Molecular Phenotyping of Human Endometrium Talbi, s. et al, "Molectlar Photyping of Human Endometiun Distinguishes Menstrual Cycle Phases and Undelying Biological Processes in Normo-Ovulatory Women," Endocrinology (Mar 2006), 147(3): 1097-1121.

Taylor, Hugh S., et al. "HOX gene expression is altered in the endometrium of women with endometriosis," Human Reproduction, 1999, vol. 14, No. 5, pp. 1328-1331.

Vierikko, P., et al. "Steroidal regulation of endometriosis tissue: lack of induction of 17 beta-hydroxysteroid dehydrogenase activity by progesterone, medroxyprogesterone acetate, or danazol" Fertil. Steril., 1985, vol. 14, No. 2, pp. 218-224.

* cited by examiner

Primary Examiner - Christopher M. Babic

(74) Attorney, Agent, or Firm - Kilpatrick Townsend \& Stockton

(57)

\section{ABSTRACT}

The present invention provides biomarkers for the diagnosis and prognosis of endometriosis. Generally, the methods of this invention find use in diagnosing or for providing a prognosis for endometriosis by detecting the expression levels of biomarkers, which are differentially expressed (up- or downregulated) in endometrial cells from a patient with endometriosis. Similarly, these markers can be used to diagnose reduced fertility in a patient with endometriosis or to provide a prognosis for a fertility trial in a patient suffering from endometriosis. The present invention also provides methods of identifying a compound for treating or preventing endometriosis. Finally, the present invention provides kits for the diagnosis or prognosis of endometriosis.

15 Claims, 26 Drawing Sheets 
patent_mesh_tag_rank_count patent_mesh_score mesh_term mesh_tree_num

143184 FEMALE

243184 HUMANS B01.050.150.900.649.313.988.400.112.400.400

342184 ENDOMETRIOSIS C13.351.500.163

410956 PROGESTERONE RESISTANCE

510955 ENDOMETRIUM A05.360.319.679.490

62940 EMBRYO IMPLANTATION G08.686.784.170.104.500

72603 CYP19A1 PROTEIN, HUMAN

82602 AROMATASE D08.811.682.690.708.170.915.099

92316 PROGESTERONE D04.210.500.745.745.654.829

101680 METHODS E05.581

101680 METHODS E05.581

111650 DIAGNOSIS E01

111650 DIAGNOSIS E01

121327 IGFBP1 PROTEIN, HUMAN

131326 INSULIN-LIKE GROWTH FACTOR BINDING PROTEIN 1 D12.776.157.420.250

141109 PELVIC PAIN C23.888.592.612.944

15866 RECEPTORS, PROGESTERONE D12.776.826.750.765

16780 UTERINE DISEASES C13.351.500.852

17683 PERITONEUM A01.923.047.025.600

18514 ESTROGENS D27.505.696.399.472.277

19467 PROLACTIN D12.644.548.691.525.525

20251 8-CHLORO-CYCLIC ADENOSINE MONOPHOSPHATE

21250 8-BROMO CYCLIC ADENOSINE MONOPHOSPHATE D13.695.667.138.395.225

22237 INFERTILITY C12.294.365

23187 CYCLIC AMP D13.695.462.200

24154 DOWN-REGULATION G05.308.200

25128 BIOMARKERS D23.101

26123 PAIN C23.888.592.612

27114 UTERUS A05.360.319.679

28101 OVARY A05.360.319.114.630

2975 APPENDIX A03.556.124.526.209.290

3072 MENSTRUATION DISTURBANCES C23.550.568

3143 LESSER PELVIS A01.923.600.500

3235 ANNEXIN A2 D12.776.157.125.050.060

3324 INSULIN D12.644.548.586.200.500.625

3423 FERTILITY G08.686.210

3520 NEOVASCULARIZATION, PATHOLOGIC C23.550.589.500

3615 FIBROBLASTS A11.329.228

3712 PHARMACEUTICAL PREPARATIONS D26

3812 INSTRUMENTATION

3910 COMPACT DISKS J01.897.280.500.668.800.250

409 SYNAPSINS D12.776.631.750

416 INVENTIONS J01.897.400

423 SOFTWARE L01.224.900

432 CENTRAL NERVOUS SYSTEM STIMULANTS D27.505.954.427.220

442 PROTEIN BINDING G02.111.679

452 DIAGNOSTIC TESTS, ROUTINE E01.370.395

461 BIOSYNTHESIS

471 METABOLISM G03 


\section{S-15.4 Example male/female-focused patent: Combinations of vasoactive agents and their use in the treatment of sexual dysfunctions US8287926}

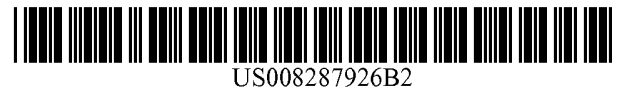

\section{(12) United States Patent} Bombardelli

(54) COMBINATIONS OF VASOACTIVE AGENTS AND THEIR USE IN THE TREATMENT OF SEXUAL DYSFUNCTIONS

(75) Inventor: Ezio Bombardelli, Milan (IT)

(73) Assignee: Indena S.p.A., Milan (IT)

(*) Notice: Subject to any disclaimer, the term of this patent is extended or adjusted under 35 U.S.C. 154(b) by 0 days.

This patent is subject to a terminal disclaimer.

(21) Appl. No.: 13/240,016

(22) Filed: Sep. 22, 2011

(65) US 2012/0009283 A1 Jan. 12, 2012

Related U.S. Application Data

(60) Continuation of application No. 12/496,194, filed on Jul. 1, 2009, which is a division of application No. $10 / 563,980$, filed as application No PCT/EP2004/007374 on Jul. 6, 2004, now abandoned.

(30) Foreign Application Priority Data

Jul. 11, 2003

(IT) MI2003A1428

(51) Int. Cl. A61K 36/16 (2006.01)

(52) U.S. Cl.

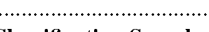

................. None See application file for complete search history.

(56)

References Cited

U.S. PATENT DOCUMENTS

$5,376,371$ A $12 / 1994$ Bombardelli

$6,306,841 \mathrm{~B} 1 *$ 10/2001 Place et al. ................... 514/149

6,514,536 B2 * 2/2003 Drizen et al. ................... 424/486

$2002 / 0013280 \mathrm{~A} 1 \quad 1 / 2002 \mathrm{Xin}$

2002/0034557 A1 3/2002 Crosby et al
(10) Patent No.: US 8,287,926 B2

(45) Date of Patent: $\quad$ *Oct. 16, 2012

Arousal Disorder. Jun. 3, 2002. Retrieved from the internet. /www. medscape.com/viewarticle/434478 $>$. Retrieved on Apr. 28, 2010. pp $1-2$.*

Drewes et al. Recent Findings on Natural Products With ErectileDysfunction Activity. Phytochemistry. 62. Apr. 2003. pp. 10191025.*

Drewes Siegfried E et al.:"Recent findings on natural products with erectile-dysfunction activity." Phytochemistry. Apr. 2003, vol. 62, No. 7, Apr. 2003, pp. 1019-1025, XP004410106 ISSN: 0031-9422 p. 1022, col. 2 .

Medscape Medical News, New Approaches to Female Sexual Arousal Disorder, Jun. 3, 2002, Retrieved from the internet http:/ www.medscape.com/viewarticle $/ 434478$, Retrieved on Apr. 28 2010, pp. 1-2.

* cited by examiner

Primary Examiner - Melenie McCormick

(74) Attorney, Agent, or Firm - Young \& Thompson

(57)

\section{ABSTRACT}

Combinations of vasoactive substances which are useful in the treatment of sexual dysfunctions associated with poor local blood supply and/or insufficient lubrication are provided. The vasoactive compounds are esculoside, visnadine, forskolin or extracts thereof, purified lipophilic extracts from plants of the genus Ipomea, esters of ximenynic acid, icarin or icarin derivatives, amentoflavone, and Gingko biloba dimeric flavones. The combination of these vasoactive substances is incorporated in gels and lotions designed to be applied to the genital organs. These formulations are useful in inducing erection of the male and female sex organs and enhancing orgasm and sexual performance. The formulations are particularly useful in the treatment of female sexual dysfunctions.

4 Claims, No Drawings 
patent_mesh_tag_rank_count patent_mesh_score mesh_term mesh_tree_num

18788 FEMALE

28788 ADULT M01.060.116

38788 MALE

48788 HUMANS B01.050.150.900.649.313.988.400.112.400.400

57788 SEXUAL DYSFUNCTION, PHYSIOLOGICAL C13.351.500.665

63059 ORGASM G08.686.784.460

72138 SEXUAL DYSFUNCTIONS, PSYCHOLOGICAL F03.835

81931 PENILE ERECTION G08.686.784.717

91173 SEXUAL BEHAVIOR F01.145.802

101065 THERAPY

101065 THERAPY

11767 COITUS F01.145.802.188

12692 SEXUAL PARTNERS M01.778

13627 SEXUALITY F01.145.802.975

14171 ACETYLCHOLINE D02.092.211.111

15161 GENITALIA, FEMALE A05.360.319

16156 CARDIOVASCULAR AGENTS D27.505.954.411

17140 GENITALIA A05.360

18111 PHYSICAL EXAMINATION E01.370.600

19108 NOREPINEPHRINE D02.092.211.215.746

2099 MUSCLE, SMOOTH A10.690.467

2197 NEUROTRANSMITTER AGENTS D27.505.519.625

2250 CALCITONIN GENE-RELATED PEPTIDE D12.644.400.097

2329 GINKGO BILOBA B01.650.940.800.575.912.125

2425 VISNADIN

2524 CHROMANS D03.633.100.150.240

2621 AMENTOFLAVONE

2720 BIFLAVONOIDS D03.383.663.283.266.450.190

2817 GINKGO BILOBA EXTRACT

2917 LUBRICATION E05.830.583

3016 PLANT EXTRACTS D26.667

3114 BLOOD CIRCULATION G09.330.100

3214 MUSCLE, STRIATED A10.690.552

3314 IPOMOEA B01.650.940.800.575.912.250.238.500

3413 FLAVONES D03.633.100.150.266.450.260

3513 XIMENYNIC ACID

3613 NITRIC OXIDE D01.625.550.500

3712 OLEIC ACIDS D10.251.355.325.600

3811 EMOTIONS F01.470

3910 COMMUNICATION F01.145.209

409 COLFORSIN D02.455.849.291.300

419 MUSCLE, SKELETAL A10.690.552.500

427 RELAXATION I03.450.769

435 MYOCYTES, SMOOTH MUSCLE A11.620.520

444 PARASYMPATHETIC NERVOUS SYSTEM A08.800.050.600

454 AUTONOMIC PATHWAYS A08.800.800.060

463 ETIOLOGY

473 CONSTITUTION AND BYLAWS N04.452.150

483 ESCULIN D09.408.348.250

492 PLANTS B01.650

502 HUMAN BODY I01.076.201.450.560

511 PSYCHOLOGY F04.096.628

521 ESTERS D02.241.400

531 INSTRUMENTATION

541 DRUG COMPOUNDING E05.916.270

551 CALCITONIN D12.644.400.095 


\section{S-16 MeSH-GBD Crosswalk}

- GBD disease cause [MeSH code] MeSH term

- Acne vulgaris [C17.800.030.150] Acne Vulgaris

- Acne vulgaris [C17.800.794.111] Acne Vulgaris

- Acute glomerulonephritis [C12.777.419.570.363] Glomerulonephritis

- Acute glomerulonephritis [C13.351.968.419.570.363] Glomerulonephritis

- Acute hepatitis A [C02.440.420] Hepatitis A

- Acute hepatitis A [C02.782.687.359.500] Hepatitis A

- Acute hepatitis A [C06.552.380.705.422] Hepatitis A

- Acute hepatitis B [C02.256.430.400] Hepatitis B

- Acute hepatitis B [C02.440.435] Hepatitis B

- Acute hepatitis B [C06.552.380.705.437] Hepatitis B

- Acute hepatitis C [C02.440.440] Hepatitis C

- Acute hepatitis C [C02.782.350.350] Hepatitis C

- Acute hepatitis C [C06.552.380.705.440] Hepatitis C

- Acute hepatitis E [C02.440.470] Hepatitis E

- Acute hepatitis E [C02.782.455] Hepatitis E

- Acute hepatitis E [C06.552.380.705.470] Hepatitis E

- Acute hepatitis [C06.552.380] Hepatitis

- African trypanosomiasis [C03.752.300.900.719] Trypanosomiasis, African

- African trypanosomiasis [C03.752.300.900] Trypanosomiasis

- Age-related and other hearing loss [C09.218.458.341] Hearing Loss

- Age-related and other hearing loss [C10.597.751.418.341] Hearing Loss

- Age-related and other hearing loss [C23.888.592.763.393.341] Hearing Loss

- Age-related macular degeneration [C11.768.585.439] Macular Degeneration

- Alcohol use disorders [C25.775.100] Alcohol-Related Disorders

- Alcohol use disorders [F03.900.100] Alcohol-Related Disorders

- Alopecia areata [C17.800.329.937.122.147] Alopecia Areata

- Alzheimer's disease and other dementias [C10.228.140.380.100] Alzheimer Disease

- Alzheimer's disease and other dementias [C10.228.140.380] Dementia

- Alzheimer's disease and other dementias [C10.574.945.249] Alzheimer Disease

- Amphetamine use disorders [C25.775.225] AmphetamineRelated Disorders

- Amphetamine use disorders [F03.900.225] AmphetamineRelated Disorders

- Anorexia nervosa [F03.400.125] Anorexia Nervosa

- Anxiety disorders [F03.080] Anxiety Disorders

- Aortic aneurysm [C14.907.055.239] Aortic Aneurysm

- Aortic aneurysm [C14.907.109.139] Aortic Aneurysm

- Appendicitis [C01.539.463.099] Appendicitis

- Appendicitis [C06.405.205.099] Appendicitis

- Appendicitis [C06.405.469.110.207] Appendicitis

- Asbestosis [C08.381.483.581.125] Asbestosis

- Asbestosis [C08.381.520.702.125] Asbestosis

- Asbestosis [C24.800.127] Asbestosis

- Ascariasis [C03.335.508.700.100.070] Ascariasis

- Asthma [C08.127.108] Asthma

- Asthma [C08.381.495.108] Asthma

- Asthma [C08.674.095] Asthma

- Asthma [C20.543.480.680.095] Asthma

- Atrial fibrillation and flutter [C14.280.067.198] Atrial Fibrillation

- Atrial fibrillation and flutter [C14.280.067.248] Atrial Flutter

- Atrial fibrillation and flutter [C23.550.073.198] Atrial Fibrillation

- Atrial fibrillation and flutter [C23.550.073.248] Atrial Flutter

- Attention-deficit/hyperactivity disorder [F03.625.094.150] Attention Deficit Disorder with Hyperactivity

- Autism spectrum disorders [F03.625.164.113] Autism Spectrum Disorder

- Bacterial skin diseases [C01.252.825] Skin Diseases, Bacterial

- Bacterial skin diseases [C01.539.800.720] Skin Diseases, Bacterial

- Bacterial skin diseases [C17.800.838.765] Skin Diseases, Bacterial

- Benign and in situ intestinal neoplasms [C04.588.274.476.411] Intestinal Neoplasms

- Benign and in situ intestinal neoplasms [C06.301.371.411] Intestinal Neoplasms

- Benign and in situ intestinal neoplasms [C06.405.249.411] Intestinal Neoplasms

- Benign and in situ intestinal neoplasms [C06.405.469.491] Intestinal Neoplasms

- Benign prostatic hyperplasia [C12.294.565.500] Prostatic Hyperplasia

- Bipolar disorder [F03.084.500] Bipolar Disorder

- Bladder cancer [C04.588.945.947.960] Urinary Bladder Neoplasms

- Bladder cancer [C12.758.820.968] Urinary Bladder Neoplasms

- Bladder cancer [C12.777.829.813] Urinary Bladder Neoplasms
- Bladder cancer [C13.351.937.820.945] Urinary Bladder Neoplasms

- Bladder cancer [C13.351.968.829.707] Urinary Bladder Neoplasms

- Blindness and vision impairment [C10.597.751.941] Vision Disorders

- Blindness and vision impairment [C11.966] Vision Disorders

- Blindness and vision impairment [C23.888.592.763.941] Vision Disorders

- Brain and nervous system cancer [C04.588.614.250.195] Brain Neoplasms

- Brain and nervous system cancer [C04.588.614] Nervous System Neoplasms

- Brain and nervous system cancer [C10.228.140.211] Brain Neoplasms

- Brain and nervous system cancer [C10.551.240.250] Brain Neoplasms

- Brain and nervous system cancer [C10.551] Nervous System Neoplasms

- Breast cancer [C04.588.180] Breast Neoplasms

- Breast cancer [C17.800.090.500] Breast Neoplasms

- Bulimia nervosa [F03.400.250] Bulimia Nervosa

- Cannabis use disorders [C25.775.635] Marijuana Abuse

- Cannabis use disorders [F01.145.610] Marijuana Use

- Cannabis use disorders [F03.900.635] Marijuana Abuse

- Cannabis use disorders [F03.900.643] Marijuana Use

- Cardiomyopathy and myocarditis [C14.280.238.625] Myocarditis

- Cardiomyopathy and myocarditis [C14.280.238] Cardiomyopathies

- Cardiovascular diseases [C14] Cardiovascular Diseases

- Cataract [C11.510.245] Cataract

- Cellulitis [C01.539.800.130] Cellulitis

- Cellulitis [C01.539.830.200] Cellulitis

- Cellulitis [C17.300.185] Cellulitis

- Cellulitis [C23.550.470.756.200] Cellulitis

- Cervical cancer [C04.588.945.418.948.850] Uterine Cervical Neoplasms

- Cervical cancer [C13.351.500.852.593.131] Uterine Cervical Neoplasms

- Cervical cancer [C13.351.500.852.762.850] Uterine Cervical Neoplasms

- Cervical cancer [C13.351.937.418.875.850] Uterine Cervical Neoplasms

- Chagas disease [C03.752.300.900.200] Chagas Disease

- Chagas disease [C03.752.300.900.200.190] Chagas Cardiomyopathy

- Chagas disease [C14.280.238.190] Chagas Cardiomyopathy

- Chlamydial infection [C01.252.400.210.210] Chlamydia Infections

- Chlamydial infection [C01.252.810.301] Chlamydia Infections

- Chlamydial infection [C01.539.778.281.301] Chlamydia Infections

- Chlamydial infection [C12.294.668.281.301] Chlamydia Infections

- Chlamydial infection [C13.351.500.711.281.301] Chlamydia Infections

- Chronic kidney disease [C05.116.198.816.750] Chronic Kidney Disease-Mineral and Bone Disorder

- Chronic kidney disease [C12.777.419.080] Chronic Kidney Disease-Mineral and Bone Disorder

- Chronic kidney disease [C13.351.968.419.795] Chronic Kidney Disease-Mineral and Bone Disorder

- Chronic kidney disease [C18.452.104.816.750] Chronic Kidney Disease-Mineral and Bone Disorder

- Chronic kidney disease [C18.452.174.845.750] Chronic Kidney Disease-Mineral and Bone Disorder

- Chronic kidney disease [C18.654.521.500.133.770.734.750] Chronic Kidney Disease-Mineral and Bone Disorder

- Chronic kidney disease [C19.642.355.480.500] Chronic Kidney Disease-Mineral and Bone Disorder

- Chronic obstructive pulmonary disease [C08.381.495.389] Pulmonary Disease, Chronic Obstructive

- Chronic respiratory diseases [C08.618] Respiration Disorders

- Cirrhosis and other chronic liver diseases [C06.552.630] Liver Cirrhosis

- Cocaine use disorders [C25.775.300] Cocaine-Related Disorders

- Cocaine use disorders [F03.900.300] Cocaine-Related Disorders

- Colon and rectum cancer [C04.588.274.476.411.307.180] Colonic Neoplasms

- Colon and rectum cancer [C04.588.274.476.411.307.790] Rectal Neoplasms

- Colon and rectum cancer [C06.301.371.411.307.180] Colonic Neoplasms

- Colon and rectum cancer [C06.301.371.411.307.790] Rectal Neoplasms

- Colon and rectum cancer [C06.405.249.411.307.180] Colonic Neoplasms

- Colon and rectum cancer [C06.405.249.411.307.790] Rectal Neo- 
plasms

- Colon and rectum cancer [C06.405.469.158.356.180] Colonic Neoplasms

- Colon and rectum cancer [C06.405.469.491.307.180] Colonic Neoplasms

- Colon and rectum cancer [C06.405.469.491.307.790] Rectal Neoplasms

- Colon and rectum cancer [C06.405.469.860.180.500] Rectal Neoplasms

- Conduct disorder [F03.625.094.300] Conduct Disorder

- Congenital birth defects [C16.131] Congenital Abnormalities

- Congenital heart anomalies [C14.240.400] Heart Defects, Congenital

- Congenital heart anomalies [C14.280.400] Heart Defects, Congenital

- Congenital heart anomalies [C16.131.240.400] Heart Defects, Congenital

- Congenital musculoskeletal and limb anomalies [N/A] N/A

- Cutaneous and mucocutaneous leishmaniasis [C03.752.300.500.400.385] Leishmaniasis, Mucocutaneous

- Cutaneous and mucocutaneous leishmaniasis [C03.858.560.400.385] Leishmaniasis, Mucocutaneous

- Cutaneous and mucocutaneous leishmaniasis [C17.800.838.775.560.400.3 Leishmaniasis, Mucocutaneous

- Cystic echinococcosis [C03.335.190.396.314] Echinococcosis, Hepatic

- Cystic echinococcosis [C03.335.190.396] Echinococcosis

- Cystic echinococcosis [C03.518.314] Echinococcosis, Hepatic

- Cystic echinococcosis [C04.182] Cysts

- Cystic echinococcosis [C06.552.664.272] Echinococcosis, Hepatic

- Cystic echinococcosis [C23.300.306] Cyst

- Cysticercosis [B01.050.500.500.736.215.895.775] Taenia solium

- Cysticercosis [C03.335.190.902.185] Cysticercosis

- Decubitus ulcer [C17.800.893.665] Pressure Ulce

- Dengue [B04.820.250.350.270] Dengue Virus

- Dengue [C02.081.270] Dengue

- Dengue [C02.782.350.250.214] Dengue

- Dengue [C02.782.417.214] Dengue

- Depressive disorders [F03.600.300] Depressive Disorder

- Dermatitis [C17.800.174] Dermatitis

- Diabetes and kidney diseases [C12.777.419] Kidney Diseases

- Diabetes and kidney diseases [C13.351.968.419] Kidney Diseases

- Diabetes and kidney diseases [C18.452.394.750] Diabetes Mellitus

- Diabetes and kidney diseases [C19.246] Diabetes Mellitus

- Diabetes mellitus [C18.452.394.750] Diabetes Mellitus

- Diabetes mellitus [C19.246] Diabetes Mellitus

- Diarrheal diseases [C06.405] Gastrointestinal Diseases

- Diarrheal diseases [C23.888.821.214] Diarrhea

- Dietary iron deficiency [C15.378.071.196.300] Anemia, IronDeficiency

- Dietary iron deficiency [C18.452.565.100] Anemia, IronDeficiency

- Digestive congenital anomalies [C16.131.314] Digestive System Abnormalities

- Digestive diseases [C06] Digestive System Diseases

- Diphtheria [C01.252.410.040.246.388] Diphtheria

- Diphtheria [D20.215.894.691.263] Diphtheria Toxoid

- Down syndrome [C10.597.606.360.220] Down Syndrome

- Down syndrome [C16.131.077.327] Down Syndrome

- Down syndrome [C16.131.260.260] Down Syndrome

- Down syndrome [C16.320.180.260] Down Syndrome

- Drug use disorders [C25.775.383] Drug Overdose

- Drug use disorders [E02.319.306.500.500] Drug Overdose

- Drug-susceptible tuberculosis [N/A] N/A

- Dysthymia [F03.600.300.400] Dysthymic Disorde

- Eating disorders [F03.400] Feeding and Eating Disorders

- Ebola [B04.820.455.300.200] Ebolaviru

- Ebola [C02.782.417.415] Hemorrhagic Fever, Ebola

- Ebola [C02.782.580.250.400] Hemorrhagic Fever, Ebola

- Ectopic pregnancy [C13.703.733] Pregnancy, Ectopic

- Encephalitis [C02.182.525] Encephalitis, Viral

- Encephalitis [C02.290] Encephalitis, Viral

- Encephalitis [C10.228.140.430.520.750] Encephalitis, Viral

- Encephalitis [C10.228.140.430] Encephalitis

- Encephalitis [C10.228.228.245.340] Encephalitis, Viral

- Encephalitis [C10.228.228.399.750] Encephalitis, Viral

- Endocarditis [C14.280.282] Endocarditis

- Endocrine, metabolic, blood, and immune disorders [C15.378] Hematologic Diseases

- Endocrine, metabolic, blood, and immune disorders [C18.452] Metabolic Diseases

- Endocrine, metabolic, blood, and immune disorders [C19] Endocrine System Diseases

- Endocrine, metabolic, blood, and immune disorders [C20] Immune System Disease

- Endometriosis [C13.351.500.163] Endometriosis

- Enteric infections [C06.405.469] Intestinal Diseases

- Epilepsy [C10.228.140.490] Epilepsy

- Esophageal cancer [C04.588.274.476.205] Esophageal Neoplasms
- Esophageal cancer [C04.588.443.353] Esophageal Neoplasms

- Esophageal cancer [C06.301.371.205] Esophageal Neoplasms

- Esophageal cancer [C06.405.117.430] Esophageal Neoplasms

- Esophageal cancer [C06.405.249.205] Esophageal Neoplasms

- Extensively drug-resistant tuberculosis [C01.252.410.040.552.846.775.500]

Extensively Drug-Resistant Tuberculosis

- Female infertility [C13.351.500.365.700] Infertility, Female

- Food-borne trematodiases [C03.335.865] Trematode Infections

- Fungal skin diseases [C01.703] Mycoses

G6PD deficiency [C15.378.071.141.150.480] Glucosephosphate Dehydrogenase Deficiency

- G6PD deficiency [C16.320.070.480] Glucosephosphate Dehydrogenase Deficiency

- G6PD deficiency [C16.320.565.202.402] Glucosephosphate Dehydrogenase Deficiency

- G6PD deficiency [C18.452.648.202.402] Glucosephosphate Dehydrogenase Deficiency

- G6PD trait [C15.378.071.141.150.480] Glucosephosphate Dehydrogenase Deficiency

- G6PD trait [C16.320.070.480] Glucosephosphate Dehydrogenase Deficiency

- G6PD trait [C16.320.565.202.402] Glucosephosphate Dehydro-

genase Deficiency

- G6PD trait [C18.452.648.202.402] Glucosephosphate Dehydrogenase Deficiency

- Gallbladder and biliary diseases [C06.130.564] Gallbladder Dis-

- Gallbladder and biliary diseases [C06.130] Biliary Tract Diseases

- Gallbladder and biliary tract cancer [C04.588.274.120.401] Gallbladder Neoplasms

- Gallbladder and biliary tract cancer [C04.588.274.120] Biliary Tract Neoplasms

- Gallbladder and biliary tract cancer [C06.130.320.401] Gallbladder Neoplasms

- Gallbladder and biliary tract cancer [C06.130.320] Biliary Tract Neoplasms

- Gallbladder and biliary tract cancer [C06.130.564.401] Gallbladder Neoplasms

- Gallbladder and biliary tract cancer [C06.301.120.401] Gallbladder Neoplasms

- Gallbladder and biliary tract cancer [C06.301.120] Biliary Tract Neoplasms

- Gastritis and duodenitis [C06.405.205.462.249] Duodenitis

- Gastritis and duodenitis [C06.405.205.697] Gastritis

- Gastritis and duodenitis [C06.405.469.275.600] Duodenitis

- Gastritis and duodenitis [C06.405.469.326.750] Duodenitis

- Gastritis and duodenitis [C06.405.748.398] Gastritis

- Gastroesophageal reflux disease [C06.405.117.119.500.484] Gastroesophageal Reflux

- Genital herpes [C02.256.466.382.290] Herpes Genitalis

- Genital herpes [C02.800.801.350] Herpes Genitalis

- Genital herpes [C12.294.329] Herpes Genitalis

- Genital herpes [C13.351.500.342] Herpes Genitalis

- Genital prolapse [C13.351.500.852.833] Uterine Prolapse

- Genital prolapse [C23.300.842.624.750] Uterine Prolapse

- Glaucoma [C11.525.381] Glaucoma

- Gonococcal infection [B03.440.400.425.550.550.474] Neisseria gonorrhoeae

- Gonococcal infection [B03.660.075.525.520.400] Neisseria gonorrhoeae

- Gonococcal infection [C01.252.400.625.391] Gonorrhea

- Gonococcal infection [C01.252.810.401] Gonorrhea

- Gonococcal infection [C01.539.778.281.401] Gonorrhea

- Gonococcal infection [C12.294.668.281.401] Gonorrhea

- Gonococcal infection [C13.351.500.711.281.401] Gonorrhea

- Gout [C05.550.114.423] Gout

- Gout [C05.550.354.500] Gout

- Gout [C05.799.414] Gout

- Gout [C16.320.565.798.368] Gout

- Gout [C18.452.648.798.368] Gout

- Guinea worm disease [B01.050.500.500.294.400.937.225.250.250] Dracunculus Nematode

- Guinea worm disease [C03.335.508.700.750.299] Dracunculiasis

- Gynecological diseases [C13.351.500] Genital Diseases, Female

- H influenzae type B meningitis [B03.440.450.600.450.330.150] Haemophilus influenzae type b

- $\mathrm{H}$ influenzae type B meningitis [B03.660.250.550.290.330.150] Haemophilus influenzae type b

- H influenzae type B meningitis [C01.252.200.500.450] Meningitis, Haemophilus

- $\mathrm{H}$ influenzae type $\mathrm{B}$ meningitis [C01.252.400.700.433.615] Meningitis, Haemophilus

- H influenzae type B meningitis [C10.228.228.180.500.425] Meningitis, Haemophilus

- H influenzae type B meningitis [C10.228.614.280.393] Meningitis, Haemophilus

- HIV/AIDS [C02.782.815.616.400.040] Acquired Immunodeficiency Syndrome

- HIV/AIDS [C02.782.815.616.400] HIV Infections

- HIV/AIDS [C02.800.801.400.040] Acquired Immunodeficiency Syndrome 
- HIV/AIDS [C02.800.801.400] HIV Infections

- HIV/AIDS [C02.839.040] Acquired Immunodeficiency Syndrome

- HIV/AIDS [C20.673.480.040] Acquired Immunodeficiency Syndrome

- HIV/AIDS [C20.673.480] HIV Infections

- HIV/AIDS and sexually transmitted infections [C01.539.778] Sexually Transmitted Diseases

- HIV/AIDS and sexually transmitted infections [C02.782.815.616.400.040] Acquired Immunodeficiency Syndrome

- HIV/AIDS and sexually transmitted infections [C02.782.815.616.400] HIV Infections

- HIV/AIDS and sexually transmitted infections [C02.800.801.400.040] Acquired Immunodeficiency Syndrome

- HIV/AIDS and sexually transmitted infections [C02.800.801.400] HIV Infections

- HIV/AIDS and sexually transmitted infections [C02.800] Sexually Transmitted Diseases

- HIV/AIDS and sexually transmitted infections [C02.839.040] Acquired Immunodeficiency Syndrome

- HIV/AIDS and sexually transmitted infections [C12.294.668] Sexually Transmitted Diseases

- HIV/AIDS and sexually transmitted infections [C13.351.500.711] Sexually Transmitted Diseases

- HIV/AIDS and sexually transmitted infections [C20.673.480.040] Acquired Immunodeficiency Syndrome

- HIV/AIDS and sexually transmitted infections [C20.673.480] HIV Infections

- Headache disorders [C10.228.140.546] Headache Disorders

- Hemoglobinopathies and hemolytic anemias [C15.378.071.141] Anemia, Hemolytic

- Hemoglobinopathies and hemolytic anemias [C15.378.420] Hemoglobinopathies

- Hemoglobinopathies and hemolytic anemias [C16.320.365] Hemoglobinopathies

- Hemolytic disease and other neonatal jaundice [C16.614.451.500] Jaundice, Neonatal

- Hemolytic disease and other neonatal jaundice [C23.550.429.249.500] Jaundice, Neonatal

- Hodgkin lymphoma [C04.557.386.355] Hodgkin Disease

- Hodgkin lymphoma [C15.604.515.569.355] Hodgkin Disease

- Hodgkin lymphoma [C20.683.515.761.355] Hodgkin Disease

- Hookworm disease [B01.050.500.500.294.400.968.100] Ancylostomatoidea

- Hookworm disease [C03.335.508.700.775.455] Hookworm Infections

- Hypertensive heart disease [C14.907.489] Hypertension

- Idiopathic developmental intellectual disability [C10.597.606.360] Intellectual Disability

- Idiopathic developmental intellectual disability [C23.888.592.604.646] Intellectual Disability

- Idiopathic developmental intellectual disability [F01.700.687] Intellectual Disability

- Idiopathic developmental intellectual disability [F03.625.539] Intellectual Disability

- Indirect maternal deaths [C13.703.401] Maternal Death

- Indirect maternal deaths [C23.550.260.730.500] Maternal Death

- Indirect maternal deaths [I01.880.735.607.500] Maternal Death

- Inflammatory bowel disease [C06.405.205.731] Inflammatory Bowel Diseases

- Inflammatory bowel disease [C06.405.469.432] Inflammatory Bowel Diseases

- Inguinal, femoral, and abdominal hernia [C23.300.707.374.750] Hernia, Femoral

- Inguinal, femoral, and abdominal hernia [C23.300.707.374.875] Hernia, Inguinal

- Inguinal, femoral, and abdominal hernia [C23.300.707.374] Hernia, Abdominal

- Interstitial lung disease and pulmonary sarcoidosis [C08.381.483.725] Sarcoidosis, Pulmonary

- Interstitial lung disease and pulmonary sarcoidosis [C08.381.483] Lung Diseases, Interstitial

- Interstitial lung disease and pulmonary sarcoidosis [C15.604.515.827.725] Sarcoidosis, Pulmonary

- Intestinal nematode infections [C03.335.508] Nematode Infections

- Intestinal nematode infections [C03.432] Intestinal Diseases, Parasitic

- Intestinal nematode infections [C06.405.469.452] Intestinal Diseases, Parasitic

- Invasive Non-typhoidal Salmonella (iNTS) [B03.440.450.425.800] Salmonella

- Invasive Non-typhoidal Salmonella (iNTS) [B03.660.250.150.710] Salmonella

- Invasive Non-typhoidal Salmonella (iNTS) [C01.252.400.310.821] Salmonella Infections

- Iodine deficiency $[\mathrm{N} / \mathrm{A}] \mathrm{N} / \mathrm{A}$

- Ischemic heart disease [C14.280.647] Myocardial Ischemia

- Ischemic heart disease [C14.907.585] Myocardial Ischemia

- Kidney cancer [C04.588.945.947.535] Kidney Neoplasms

- Kidney cancer [C12.758.820.750] Kidney Neoplasms
- Kidney cancer [C12.777.419.473] Kidney Neoplasms

- Kidney cancer [C13.351.937.820.535] Kidney Neoplasms

- Kidney cancer [C13.351.968.419.473] Kidney Neoplasms

- Klinefelter syndrome [C16.131.260.830.835.500] Klinefelter Syndrome

- Klinefelter syndrome [C16.131.939.316.795.500] Klinefelter Syndrome

- Klinefelter syndrome [C16.320.180.830.835.500] Klinefelter Syndrome

- Larynx cancer [C04.588.443.665.481] Laryngeal Neoplasms

- Larynx cancer [C08.360.369] Laryngeal Neoplasms

- Larynx cancer [C08.785.481] Laryngeal Neoplasms

- Larynx cancer [C09.400.369] Laryngeal Neoplasms

- Larynx cancer [C09.647.481] Laryngeal Neoplasms

- Late maternal deaths [C13.703.401] Maternal Death

- Late maternal deaths [C13.703.401] Maternal Death

- Late maternal deaths,I01.880.735.607.500] Maternal Death

- Latent tuberculosis infection [C01.252.410.040.552.846.122] Latent Tuberculosis

- Leishmaniasis [C03.752.300.500] Leishmaniasis

- Leishmaniasis [C03.858.560] Leishmaniasis

- Leishmaniasis [C17.800.838.775.560] Leishmaniasis

- Leprosy [C01.252.410.040.552.386] Leprosy

- Leukemia [C04.557.337] Leukemia

- Lip and oral cavity cancer [C04.588.443.591.550] Lip Neoplasms

- Lip and oral cavity cancer [C04.588.443.591] Mouth Neoplasms

- Lip and oral cavity cancer [C07.465.409.640] Lip Neoplasms

- Lip and oral cavity cancer [C07.465.530.550] Lip Neoplasms

- Lip and oral cavity cancer [C07.465.530] Mouth Neoplasms

- Liver cancer [C04.588.274.623] Liver Neoplasms

- Liver cancer [C06.301.623] Liver Neoplasms

- Liver cancer [C06.552.697] Liver Neoplasms

- Low back pain [C23.888.592.612.107.400] Low Back Pain

- Lower respiratory infections [C01.539.739] Respiratory Tract Infections

- Lower respiratory infections [C08.730] Respiratory Tract Infections

- Lymphatic filariasis [C03.335.508.700.750.361.350] Elephantiasis, Filarial

- Lymphatic filariasis [C03.335.508.700.750.361] Filariasis

- Lymphatic filariasis [C15.604.496.490] Elephantiasis, Filarial

- Major depressive disorder [F03.600.300.375] Depressive Disorder, Major

- Malaria [C03.752.530] Malaria

- Male infertility [C12.294.365.700] Infertility, Male

- Malignant skin melanoma [C04.557.465.625.650.510] Melanoma

- Malignant skin melanoma [C04.557.580.625.650.510] Melanoma

- Malignant skin melanoma [C04.557.665.510] Melanoma

- Maternal abortion and miscarriage [C13.703.039] Abortion, Spontaneous

- Maternal abortion and miscarriage [C13.703.090] Abortion, Threatened

- Maternal and neonatal disorders [N/A] N/A

- Maternal deaths aggravated by HIV/AIDS [C13.703.401] Maternal Death

- Maternal deaths aggravated by HIV/AIDS [C23.550.260.730.500] Maternal Death

- Maternal deaths aggravated by HIV/AIDS [I01.880.735.607.500] Maternal Death

- Maternal disorders [C13.703] Pregnancy Complications

- Maternal hemorrhage [C13.703.420.725] Postpartum Hemorrhage

- Maternal hemorrhage [C13.703.844.700] Postpartum Hemorrhage

- Maternal hemorrhage [C23.550.414.993.850] Postpartum Hemorrhage

- Maternal hypertensive disorders [C13.703.395] Hypertension, Pregnancy-Induced

- Maternal hypertensive disorders [C14.907.489.480] Hypertension, Pregnancy-Induced

- Maternal obstructed labor and uterine rupture [C13.351.500.852.904] Uterine Rupture

- Maternal obstructed labor and uterine rupture [C13.703.420] Obstetric Labor Complications

- Maternal sepsis and other maternal infections [C01.539.674] Pregnancy Complications, Infectious

- Maternal sepsis and other maternal infections [C13.703.700] Pregnancy Complications, Infectious

- Measles [C02.782.580.600.500.500] Measles

- Meningitis [C01.252.200.500] Meningitis, Bacterial

- Meningitis [C01.703.181.500] Meningitis, Fungal

- Meningitis [C02.182.550] Meningitis, Viral

- Meningitis [C10.228.140.430.550] Meningoencephalitis

- Meningitis [C10.228.228.180.500] Meningitis, Bacterial

- Meningitis [C10.228.228.198.500] Meningitis, Fungal

- Meningitis [C10.228.228.245.500] Meningitis, Viral

- Meningitis [C10.228.228.245.550] Meningoencephalitis

- Meningitis [C10.228.228.570] Meningoencephalitis

- Meningitis [C10.228.614.220] Meningitis, Aseptic

- Meningitis [C10.228.614.280] Meningitis, Bacterial

- Meningitis [C10.228.614.300] Meningitis, Fungal

- Meningitis [C10.228.614.400] Meningitis, Viral 
- Meningitis [C10.228.614.500] Meningoencephalitis

- Meningitis [C10.228.614] Meningitis

- Meningococcal meningitis [C01.252.200.500.550] Meningitis, Meningococcal

- Meningococcal meningitis [C01.252.400.625.549.449] Meningitis, Meningococcal

- Meningococcal meningitis [C10.228.228.180.500.750] Meningitis, Meningococcal

- Meningococcal meningitis [C10.228.614.280.505] Meningitis, Meningococcal

- Mental disorders [F03] Mental Disorders

- Mesothelioma [C04.557.470.035.510] Mesothelioma

- Mesothelioma [C04.557.470.660.510] Mesothelioma

- Migraine [C10.228.140.546.399.750] Migraine Disorders

- Motor neuron disease [C10.574.562] Motor Neuron Disease

- Motor neuron disease [C10.668.467] Motor Neuron Disease

- Multidrug-resistant tuberculosis without extensive drug resistance [C01.252.410.040.552.846.775] Tuberculosis, MultidrugResistant

- Multiple myeloma [C04.557.595.500] Multiple Myeloma

- Multiple myeloma [C14.907.454.460] Multiple Myeloma

- Multiple myeloma [C15.378.147.780.650] Multiple Myeloma

- Multiple myeloma [C15.378.463.515.460] Multiple Myeloma

- Multiple myeloma [C20.683.515.845] Multiple Myeloma

- Multiple myeloma [C20.683.780.650] Multiple Myeloma

- Multiple sclerosis [C10.114.375.500] Multiple Sclerosis

- Multiple sclerosis [C10.314.350.500] Multiple Sclerosis

- Multiple sclerosis [C20.111.258.250.500] Multiple Sclerosis

- Musculoskeletal disorders [C05] Musculoskeletal Diseases

- Myelodysplastic, myeloproliferative, and other hematopoietic neoplasms [C04.588.448] Hematologic Neoplasms

- Myelodysplastic, myeloproliferative, and other hematopoietic neoplasms [C15.378.400] Hematologic Neoplasms

- Nasopharynx cancer [C04.588.443.665.710.650] Nasopharyngeal Neoplasms

- Nasopharynx cancer [C07.550.350.650] Nasopharyngeal Neoplasms

- Nasopharynx cancer [C07.550.745.650] Nasopharyngeal Neoplasms

- Nasopharynx cancer [C09.647.710.650] Nasopharyngeal Neoplasms

- Nasopharynx cancer [C09.775.350.650] Nasopharyngeal Neoplasms

Nasopharynx cancer [C09.775.549.650] Nasopharyngeal Neoplasms

- Near vision loss [C11.744.786] Presbyopia

- Neck pain [C23.888.592.612.553] Neck Pain

- Neglected tropical diseases and malaria [N/A] N/A

- Neonatal disorders [C16.614] Infant, Newborn, Diseases

- Neonatal disorders [C16] Congenital, Hereditary, and Neonatal Diseases and Abnormalities

- Neonatal encephalopathy due to birth asphyxia and trauma [C16.614.092] Asphyxia Neonatorum

- Neonatal preterm birth [C13.703.420.491.500] Premature Birth

- Neonatal sepsis and other neonatal infections [C01.539.757.580] Neonatal Sepsis

- Neonatal sepsis and other neonatal infections [C16.614.627] Neonatal Sepsis

- Neonatal sepsis and other neonatal infections [C23.550.470.790.500.470] Neonatal Sepsis

Neoplasms [C04] Neoplasms

- Neural tube defects [C10.500.680] Neural Tube Defects

- Neural tube defects [C16.131.666.680] Neural Tube Defects

- Neurological disorders [C10] Nervous System Diseases

- Non-Hodgkin lymphoma [C04.557.386.480] Lymphoma, NonHodgkin

- Non-Hodgkin lymphoma [C15.604.515.569.480] Lymphoma, Non-Hodgkin

- Non-Hodgkin lymphoma [C20.683.515.761.480] Lymphoma, Non-Hodgkin

- Non-melanoma skin cancer [C04.588.805] Skin Neoplasms

- Non-melanoma skin cancer [C17.800.882] Skin Neoplasms

- Non-rheumatic valvular heart disease [C14.280.484] Heart Valve Diseases

- Nutritional deficiencies [C18.654.521] Malnutrition

- Onchocerciasis [C03.335.508.700.750.361.699] Onchocerciasis

- Onchocerciasis [C03.858.650] Onchocerciasis

- Onchocerciasis [C17.800.838.775.690] Onchocerciasis

- Opioid use disorders [C25.775.675] Opioid-Related Disorder

- Opioid use disorders [F03.900.675] Opioid-Related Disorders

- Oral disorders [C07.793] Tooth Diseases

- Orofacial clefts [N/A] N/A

- Osteoarthritis [C05.550.114.606] Osteoarthritis

- Osteoarthritis [C05.799.613] Osteoarthritis

- Other chromosomal abnormalities [C16.131.260] Chromosome Disorders

- Other chromosomal abnormalities [C16.320.180] Chromosome Disorders

- Other non-communicable diseases [N/A] N/A

- Other pharynx cancer [C04.588.443.665.710] Pharyngeal Neoplasms

- Other pharynx cancer [C07.550.745] Pharyngeal Neoplasms
- Other pharynx cancer [C09.647.710] Pharyngeal Neoplasms - Other pharynx cancer [C09.775.549] Pharyngeal Neoplasms - Otitis media [C09.218.705.663] Otitis Media

- Ovarian cancer [C04.588.322.455] Ovarian Neoplasms

- Ovarian cancer [C13.351.500.056.630.705] Ovarian Neoplasms

- Ovarian cancer [C13.351.937.418.685] Ovarian Neoplasms

- Ovarian cancer [C19.344.410] Ovarian Neoplasms

- Ovarian cancer [C19.391.630.705] Ovarian Neoplasms

- Pancreatic cancer [C04.588.274.761] Pancreatic Neoplasms

- Pancreatic cancer [C04.588.322.475] Pancreatic Neoplasms

- Pancreatic cancer [C06.301.761] Pancreatic Neoplasms

- Pancreatic cancer [C06.689.667] Pancreatic Neoplasms

- Pancreatic cancer [C19.344.421] Pancreatic Neoplasms

- Pancreatitis [C06.689.750] Pancreatitis

- Paralytic ileus and intestinal obstruction [C06.405.469.531.492.500] Intestinal Pseudo-Obstruction

- Paralytic ileus and intestinal obstruction [C06.405.469.531] Intestinal Obstruction

- Paratyphoid fever [C01.252.400.310.821.438] Paratyphoid Fever

- Parkinson's disease [C10.228.140.079.862.500] Parkinson Disease

- Parkinson's disease [C10.228.662.600.400] Parkinson Disease

- Parkinson's disease [C10.574.812] Parkinson Disease

- Peptic ulcer disease [C06.405.469.275.800] Peptic Ulcer

- Peptic ulcer disease [C06.405.748.586] Peptic Ulce

- Peripheral artery disease [C14.907.137.126.307.500] Peripheral Arterial Disease

- Peripheral artery disease [C14.907.617.671] Peripheral Arterial Disease

- Pneumococcal meningitis [C01.252.200.500.600] Meningitis, Pneumococcal

- Pneumococcal meningitis [C01.252.410.890.670.595] Meningitis, Pneumococcal

- Pneumococcal meningitis [C10.228.228.180.500.875] Meningitis, Pneumococcal

- Pneumococcal meningitis [C10.228.614.280.560] Meningitis, Pneumococcal

- Pneumoconiosis [C08.381.483.581] Pneumoconiosis

- Pneumoconiosis [C08.381.520.702] Pneumoconiosis

- Pneumoconiosis [C24.800] Pneumoconiosis

- Polycystic ovarian syndrome [C04.182.612.765] Polycystic Ovary Syndrome

- Polycystic ovarian syndrome [C13.351.500.056.630.580.765] Polycystic Ovary Syndrome

- Polycystic ovarian syndrome [C19.391.630.580.765] Polycystic Ovary Syndrome

- Premenstrual syndrome [C23.550.568.968] Premenstrual Syndrome

- Prostate cancer [C04.588.945.440.770] Prostatic Neoplasms

- Prostate cancer [C12.294.260.750] Prostatic Neoplasms

- Prostate cancer [C12.294.565.625] Prostatic Neoplasms

- Prostate cancer [C12.758.409.750] Prostatic Neoplasms

- Protein-energy malnutrition [C18.654.521.500.708.626] ProteinEnergy Malnutrition

- Pruritus [C17.800.685] Pruritus

- Pruritus [C23.888.885.625] Pruritus

- Psoriasis [C17.800.859.675] Psoriasis

- Pyoderma [C17.800.695] Pyoderma

- Rabies [B04.820.455.750.500.700] Rabies virus

- Rabies [C02.782.580.830.750] Rabies

- Refraction disorders [C11.744] Refractive Errors

- Respiratory infections and tuberculosis [C01.252.410.040.552.846] Tuberculosis

- Respiratory infections and tuberculosis [C01.539.739] Respiratory Tract Infections

- Respiratory infections and tuberculosis [C08.730] Respiratory Tract Infections

- Rheumatic heart disease [C01.252.410.890.731.649] Rheumatic Heart Disease

- Rheumatic heart disease [C14.280.874] Rheumatic Heart Disease

- Rheumatoid arthritis [C05.550.114.154] Arthritis, Rheumatoid

- Rheumatoid arthritis [C05.799.114] Arthritis, Rheumatoid

- Rheumatoid arthritis [C17.300.775.099] Arthritis, Rheumatoid

- Rheumatoid arthritis [C20.111.199] Arthritis, Rheumatoid

- Scabies [C03.858.211.480.708] Scabies

- Scabies [C17.800.838.775.800] Scabies

- Schistosomiasis [C03.335.865.859] Schistosomiasis

- Schizophrenia [F03.700.750] Schizophrenia

- Schizophrenia [F04.824] Schizophrenic Psychology

- Sense organ diseases [C10.597.751] Sensation Disorders

- Sense organ diseases [C23.888.592.763] Sensation Disorders

- Sickle cell disorders [C15.378.071.141.150.150] Anemia, Sickle Cell

- Sickle cell disorders [C15.378.420.155] Anemia, Sickle Cell

- Sickle cell disorders [C16.320.070.150] Anemia, Sickle Cell

- Sickle cell disorders [C16.320.365.155] Anemia, Sickle Cell

- Sickle cell trait [C15.378.071.141.150.150.670] Sickle Cell Trait

- Sickle cell trait [C15.378.420.155.668] Sickle Cell Trait

- Sickle cell trait [C16.320.070.150.670] Sickle Cell Trait

- Sickle cell trait [C16.320.365.155.668] Sickle Cell Trait

- Silicosis [C08.381.483.581.760] Silicosis 
- Silicosis [C08.381.520.702.760] Silicosis

- Silicosis [C24.800.834] Silicosis

- Skin and subcutaneous diseases [C17.800] Skin Diseases

- Stomach cancer [C04.588.274.476.767] Stomach Neoplasms

- Stomach cancer [C06.301.371.767] Stomach Neoplasms

- Stomach cancer [C06.405.249.767] Stomach Neoplasms

- Stomach cancer [C06.405.748.789] Stomach Neoplasms

- Stroke [C10.228.140.300.775] Stroke

- Stroke [C14.907.253.855] Stroke

- Substance use disorders [C25.775] Substance-Related Disorder

- Substance use disorders [F03.900] Substance-Related Disorders

- Sudden infant death syndrome [C23.550.260.322.625] Sudden Infant Death

- Sudden infant death syndrome [C23.550.260.657.500] Sudden Infant Death

- Syphilis [C01.252.400.840.744] Syphilis

- Syphilis [C01.252.810.859] Syphilis

- Syphilis [C01.252.847.840.744] Syphilis

- Syphilis [C01.539.778.281.859] Syphilis

- Syphilis [C12.294.668.281.859] Syphilis

- Syphilis [C13.351.500.711.281.859] Syphilis

- Tension-type headache [C10.228.140.546.399.875] Tension-Type Headache

- Testicular cancer [C04.588.322.762] Testicular Neoplasms

- Testicular cancer [C04.588.945.440.915] Testicular Neoplasms

- Testicular cancer [C12.294.260.937] Testicular Neoplasms

- Testicular cancer [C12.758.409.937] Testicular Neoplasms

- Testicular cancer [C19.344.762] Testicular Neoplasms

- Testicular cancer [C19.391.829.782] Testicular Neoplasms

- Tetanus [C01.252.410.222.864] Tetanus

- Tetanus [D20.215.894.691.824] Tetanus Toxoid

- Thalassemias [C15.378.071.141.150.875] Thalassemia

- Thalassemias [C15.378.420.826] Thalassemia

- Thalassemias [C16.320.070.875] Thalassemia

- Thalassemias [C16.320.365.826] Thalassemia

- Thalassemias trait [C15.378.071.141.150.875.150] betaThalassemia

- Thalassemias trait [C15.378.420.826.150] beta-Thalassemia

- Thalassemias trait [C16.320.070.875.150] beta-Thalassemia

- Thalassemias trait [C16.320.365.826.150] beta-Thalassemia

- Thyroid cancer [C04.588.322.894] Thyroid Neoplasms

- Thyroid cancer [C04.588.443.915] Thyroid Neoplasms

- Thyroid cancer [C19.344.894] Thyroid Neoplasms

- Thyroid cancer [C19.874.788] Thyroid Neoplasms

- Tracheal, bronchus, and lung cancer [C04.588.443.925] Tracheal Neoplasms

- Tracheal, bronchus, and lung cancer [C04.588.894.797.520.109] Bronchial Neoplasms

- Tracheal, bronchus, and lung cancer [C04.588.894.797.520] Lung Neoplasms

- Tracheal, bronchus, and lung cancer [C04.588.894.797.760] Tracheal Neoplasms

- Tracheal, bronchus, and lung cancer [C08.127.265] Bronchial Neoplasms

- Tracheal, bronchus, and lung cancer [C08.381.540] Lung Neoplasms

- Tracheal, bronchus, and lung cancer [C08.785.520.100] Bronchial Neoplasms

- Tracheal, bronchus, and lung cancer [C08.785.520] Lung Neoplasms

- Tracheal, bronchus, and lung cancer [C08.785.760] Tracheal Neoplasms

- Tracheal, bronchus, and lung cancer [C08.907.563] Tracheal Neoplasms

- Trachoma [C01.252.354.225.800] Trachoma
- Trachoma [C01.252.400.210.210.800] Trachoma

- Trachoma [C01.539.375.354.220.800] Trachom

- Trachoma [C11.187.183.220.889] Trachoma

- Trachoma [C11.204.813] Trachoma

- Trachoma [C11.294.354.220.800] Trachoma

- Trichomoniasis [B01.630.800.808.717] Trichomonas vaginalis

- Trichomoniasis [C03.752.890] Trichomonas Infections

- Trichuriasis [C03.335.508.100.275.895] Trichuriasis

- Tuberculosis [C01.252.410.040.552.846] Tuberculosis

- Turner syndrome [C16.131.240.400.970] Turner Syndrome

- Turner syndrome [C16.131.260.830.835.750] Turner Syndrome

- Turner syndrome [C16.131.939.316.309.872] Turner Syndrome

- Turner syndrome [C16.131.939.316.795.750] Turner Syndrome

- Turner syndrome [C16.320.180.830.835.750] Turner Syndrome

- Typhoid and paratyphoid [C01.252.400.310.821.438] Paratyphoid Fever

- Typhoid and paratyphoid [C01.252.400.310.821.873] Typhoid Fever

- Typhoid fever [C01.252.400.310.821.873] Typhoid Fever

- Upper digestive system diseases [N/A] N/A

- Upper respiratory infections [C01.539.739] Respiratory Tract Infections

- Upper respiratory infections [C08.730] Respiratory Tract Infections

- Urinary diseases and male infertility [C12.294.365.700] Infertility, Male

- Urinary diseases and male infertility [C12.777] Urologic Diseases

- Urinary diseases and male infertility [C13.351.968] Urologic Diseases

- Urinary tract infections [C01.539.895] Urinary Tract Infections

- Urinary tract infections [C12.777.892] Urinary Tract Infections

- Urinary tract infections [C13.351.968.892] Urinary Tract Infections

- Urogenital congenital anomalies [C16.131.939] Urogenital Abnormalities

- Urolithiasis [C12.777.967] Urolithiasis

- Urolithiasis [C13.351.968.967] Urolithiasis

- Urticaria [C17.800.862.945] Urticaria

- Urticaria [C20.543.480.904] Urticaria

- Uterine cancer [C04.588.945.418.948] Uterine Neoplasms

- Uterine cancer [C04.588.945.418.948] Uterine Neoplasms

- Uterine cancer [C13.351.937.418.875] Uterine Neoplasms

- Uterine fibroids [C04.557.450.590.450] Leiomyoma

- Varicella and herpes zoster [B04.280.382.100.900.460] Herpesvirus 3] Human

- Varicella and herpes zoster [C02.256.466.930.250] Chickenpox

- Varicella and herpes zoster [C02.256.466.930.750] Herpes Zoster

- Vascular intestinal disorders [C14.907] Vascular Diseases

- Viral skin diseases [C02.825] Skin Diseases, Viral

- Viral skin diseases [C02.825] Skin Diseases, Viral

- Visceral leishmaniasis [C03.752.300.500.510] Leishmaniasis, Visceral

- Vitamin A deficiency [C18.654.521.500.133.628] Vitamin A Deficiency

- Whooping cough [C01.252.400.143.740] Whooping Cough

- Whooping cough [C01.539.739.969] Whooping Cough

- Whooping cough [C08.730.969] Whooping Cough

- Yellow fever [B04.820.250.350.990] Yellow fever virus

- Yellow fever [C02.081.980] Yellow Fever

- Yellow fever [C02.782.350.250.980] Yellow Fever

- Yellow fever [C02.782.417.881] Yellow Fever

- Zika virus [B04.820.250.350.995] Zika Virus

- Zika virus [C02.081.990] Zika Virus Infection

- Zika virus [C02.782.350.250.990] Zika Virus Infection

Table S-1: Estimates showing the percentage of patents classified as female-focused by the MTI algorithm that are classified as female-focused by trained MeSH indexers. 95\% Confidence intervals in brackets. Top left shows recall, bottom left the false negative rate, bottom right the precision, and top right the false positive rate.

\begin{tabular}{c|cc}
\hline \hline \multirow{2}{*}{ Female-focused (Indexer) } & \multicolumn{2}{|c}{ Female-focused (MTI) } \\
& False & True \\
\hline False & 65.46 & 6.84 \\
& {$[55.71,74.06]$} & {$[3.73,12.21]$} \\
True & 34.54 & 93.16 \\
& {$[25.94,44.29]$} & {$[87.79,96.27]$} \\
\hline \hline
\end{tabular}


Table S-2: Estimates showing the percentage of patents classified as male-focused by the MTI algorithm that are classified as male-focused by trained MeSH indexers. 95\% Confidence intervals in brackets. Top left shows recall, bottom left the false negative rate, bottom right the precision, and top right the false positive rate.

\begin{tabular}{c|cc}
\hline \hline \multirow{2}{*}{ Male-focused (Indexer) } & \multicolumn{2}{|c}{ Male-focused (MTI) } \\
& False & True \\
\hline False & 67.43 & 7.52 \\
& {$[57.54,75.98]$} & {$[4.03,13.60]$} \\
True & 32.57 & 92.48 \\
& {$[24.02,42.46]$} & {$[86.40,95.97]$} \\
\hline \hline
\end{tabular}

Table S-3: Precision and recall for whether a patent is female-focused, split by the gender composition of the inventor team. 95\% Confidence intervals in brackets. In each table the top left shows recall, bottom left the false negative rate, bottom right the precision, and top right the false positive rate.

(A) Female Majority Team (50\% or Greater)

\begin{tabular}{c|cc}
\hline \hline \multirow{2}{*}{ Female-focused (Indexer) } & \multicolumn{2}{|c}{ Female-focused (MTI) } \\
& False & True \\
\hline False & 65.47 & 3.69 \\
& {$[53.75,75.56]$} & {$[1.56,8.49]$} \\
True & 34.53 & 96.31 \\
& {$[24.44,46.25]$} & {$[91.51,98.44]$} \\
\hline \hline
\end{tabular}

(B) Male Majority Team (Greater than 50\%)

\begin{tabular}{c|cc}
\hline \hline \multirow{2}{*}{ Female-focused (Indexer) } & \multicolumn{2}{|c}{ Female-focused (MTI) } \\
& False & True \\
\hline False & 65.46 & 7.53 \\
True & {$[54.00,75.37]$} & $[3.86,14.17)]$ \\
& 34.54 & 92.47 \\
& {$[24.63,46.00]$} & $[85.83,96.14)]$ \\
\hline \hline
\end{tabular}


Table S-4: Precision and recall for whether a patent is male-focused, split by the gender composition of the inventor team. 95\% Confidence intervals in brackets. In each table the top left shows recall, bottom left the false negative rate, bottom right the precision, and top right the false positive rate.

\begin{tabular}{c|cc} 
(A) Female Majority Team $(50 \%$ or Greater $)$ \\
\hline \multirow{2}{*}{ Male (Indexer) } & \multicolumn{2}{|c}{ Male (MTI) } \\
& False & True \\
\hline False & 70.86 & 7.48 \\
\multirow{2}{*}{ True } & {$[59.01,80.42]$} & {$[4.10,13.27]$} \\
& 29.14 & 92.52 \\
& {$[19.58,40.99]$} & {$[86.73,95.90]$} \\
\hline
\end{tabular}

(B) Male Majority Team (Greater than 50\%)

\begin{tabular}{c|cc}
\hline \hline \multirow{2}{*}{ Male (Indexer) } & \multicolumn{2}{|c}{ Male (MTI) } \\
& False & True \\
\hline False & 66.77 & 7.53 \\
& $[55.11,76.68)]$ & {$[3.63,14.97]$} \\
True & 33.23 & 92.47 \\
& {$[23.32,44.89]$} & {$[85.03,96.37]$} \\
\hline \hline
\end{tabular}


Table S-5: The 100 words most associated with patents tagged with the "Female" check tag.

\begin{tabular}{|c|c|c|c|c|c|}
\hline $\begin{array}{l}\text { Z-Score } \\
\text { Rank }\end{array}$ & Word & $\begin{array}{l}\text { Raw Odds-Ratio } \\
\text { (Weighted Z-Score) }\end{array}$ & $\begin{array}{l}\text { Z-Score } \\
\text { Rank }\end{array}$ & Word & $\begin{array}{l}\text { Raw Odds-Ratio } \\
\text { (Weighted Z-Score) }\end{array}$ \\
\hline 1 & cows & $6843.8(31)$ & 51 & vulvar & $176.6(6)$ \\
\hline 2 & gynecologic & $2113(17)$ & 52 & labia & $167.6(10)$ \\
\hline 3 & iud & $1911.2(17)$ & 53 & cesarean & $165.5(7)$ \\
\hline 4 & lactating & $1870.8(16)$ & 54 & nonoxynol & $157.5(7)$ \\
\hline 5 & hysterectomy & $1860.7(15)$ & 55 & uterus & 157.1 (38) \\
\hline 6 & sows & 1803.5 (16) & 56 & papanicolaou & $150(8)$ \\
\hline 7 & premenopausal & $1009.4(12)$ & 57 & guzmania & $147.5(10)$ \\
\hline 8 & granulosa & 948.8 (12) & 58 & hysteroscopy & $146.7(6)$ \\
\hline 9 & mares & 942.1 (12) & 59 & mammogram & $145.1(9)$ \\
\hline 10 & mastitis & $923.6(21)$ & 60 & mammographic & $141.3(8)$ \\
\hline 11 & breastpump & 847.9 (11) & 61 & clitoral & $139.4(7)$ \\
\hline 12 & girls & $821(11)$ & 62 & cow & $136(19)$ \\
\hline 13 & calving & $767.2(10)$ & 63 & interlabial & 135.9 (11) \\
\hline 14 & oestrone & 753.7 (9) & 64 & ovulation & $134.8(22)$ \\
\hline 15 & mare & $747(10)$ & 65 & pregnancies & $130.6(13)$ \\
\hline 16 & childbearing & $679.7(10)$ & 66 & endometrial & $127.2(23)$ \\
\hline 17 & breastpumps & $551.8(8)$ & 67 & menstruation & $124(15)$ \\
\hline 18 & woman & 491.8 (27) & 68 & dysmenorrhea & $123.3(13)$ \\
\hline 19 & clitoris & 474.4 (8) & 69 & progestogen & $111(9)$ \\
\hline 20 & spermicide & 437.4 (7) & 70 & msd & $107.7(8)$ \\
\hline 21 & vulva & $437.4(9)$ & 71 & megestrol & $99(6)$ \\
\hline 22 & udder & $427.3(10)$ & 72 & endometrium & 98.3 (18) \\
\hline 23 & estrus & 405.1 (11) & 73 & pcos & $97.1 \quad(6)$ \\
\hline 24 & menopause & 402.5 (22) & 74 & uteri & $94.9(7)$ \\
\hline 25 & luteal & $381.3(8)$ & 75 & teats & 90.8 (8) \\
\hline 26 & women & 378.1 (71) & 76 & menopausal & $89(22)$ \\
\hline 27 & enterocele & 376.8 (7) & 77 & gestagen & 88.2 (7) \\
\hline 28 & vagina & $348 \quad(36)$ & 78 & fibroids & 87.9 (12) \\
\hline 29 & fallopian & $305.1 \quad(22)$ & 79 & intrauterine & $84.6(18)$ \\
\hline 30 & endocervical & 287.7 (8) & 80 & brassiere & $81.3(7)$ \\
\hline 31 & vaginosis & $279.9(9)$ & 81 & uterine & $79.3(38)$ \\
\hline 32 & spermicidal & 276.7 (12) & 82 & $\operatorname{cin}$ & $78.8(10)$ \\
\hline 33 & spermicides & 275.9 (7) & 83 & oviduct & $78.7(8)$ \\
\hline 34 & postpartum & $271.4(10)$ & 84 & intravaginal & 77.9 (13) \\
\hline 35 & rectocele & 262.4 (7) & 85 & pregnancy & 77.7 (35) \\
\hline 36 & iuds & $261.1(9)$ & 86 & antiestrogen & $77.6(8)$ \\
\hline 37 & menorrhagia & $259.6(10)$ & 87 & lumpectomy & $76(6)$ \\
\hline 38 & vaginal & 257.3 (49) & 88 & hysteroscope & 74.9 (8) \\
\hline 39 & cystocele & 254.4 (9) & 89 & endometriosis & 73.1 (20) \\
\hline 40 & pessary & $252.4(9)$ & 90 & ivf & $72.5(10)$ \\
\hline 41 & cervix & 245.8 (29) & 91 & birthing & 70.7 (6) \\
\hline 42 & pregnant & $235.4(23)$ & 92 & trimester & 67.8 (7) \\
\hline 43 & females & $235.2(28)$ & 93 & mammograms & $66.8(7)$ \\
\hline 44 & smbg & $212(10)$ & 94 & mammography & $65(18)$ \\
\hline 45 & lactation & 209.1 (14) & 95 & preterm & 64.7 (14) \\
\hline 46 & postmenopausal & 207.9 (18) & 96 & breastfeeding & $64.2(7)$ \\
\hline 47 & vaginitis & $198.9(10)$ & 97 & mastectomy & 63.7 (11) \\
\hline 48 & hens & $197.4(8)$ & 98 & hypermobility & $63.2(9)$ \\
\hline 49 & amniocentesis & $187.5(9)$ & 99 & transvaginal & $62.5(7)$ \\
\hline 50 & sow & $183.4(7)$ & 100 & progestin & $62.3(13)$ \\
\hline
\end{tabular}


Table S-6: The 100 words most associated with patents tagged with the "Male" check tag.

\begin{tabular}{|c|c|c|c|c|c|}
\hline $\begin{array}{l}\text { Z-Score } \\
\text { Rank } \\
\end{array}$ & Word & $\begin{array}{l}\begin{array}{l}\text { Raw Odds-Ratio } \\
\text { (Weighted Z-Score) }\end{array} \\
\end{array}$ & $\begin{array}{c}\text { Z-Score } \\
\text { Rank }\end{array}$ & Word & $\begin{array}{l}\text { Raw Odds-Ratio } \\
\text { (Weighted Z-Score) }\end{array}$ \\
\hline 1 & prostatectomy & $3636.5(23)$ & 51 & klk & $72.2(6)$ \\
\hline 2 & cavernosa & 2008.1 (17) & 52 & urethral & $67.7(30)$ \\
\hline 3 & cavernosum & $926.8(11)$ & 53 & resectoscope & $62.2(13)$ \\
\hline 4 & vasectomy & 914 (12) & 54 & sperm & $57.8(31)$ \\
\hline 5 & retropubic & $746.6(11)$ & 55 & sexes & $49.8(9)$ \\
\hline 6 & peyronie & $740.2(10)$ & 56 & testis & $48.6(13)$ \\
\hline 7 & erections & $727.3(10)$ & 57 & pellucida & $46.7(8)$ \\
\hline 8 & penis & $556.2(39)$ & 58 & sui & 46.2 (13) \\
\hline 9 & scrotum & $512.3(12)$ & 59 & androgenetic & $45.7(7)$ \\
\hline 10 & erection & $506.4(26)$ & 60 & tumescence & $41.3(6)$ \\
\hline 11 & isd & $407.6(9)$ & 61 & phosphenes & $39.6(9)$ \\
\hline 12 & impotence & $406.7(26)$ & 62 & nocturia & $37.3(9)$ \\
\hline 13 & circumcision & $351.9(8)$ & 63 & orgasm & $36.8(8)$ \\
\hline 14 & spermatozoa & $346.9(15)$ & 64 & androgen & $33.1 \quad(30)$ \\
\hline 15 & ejaculation & $335.3(14)$ & 65 & testicles & $32.5(6)$ \\
\hline 16 & penile & $330.7(30)$ & 66 & testosterone & $32.2(26)$ \\
\hline 17 & bull & $286.6(24)$ & 67 & testicular & $32.2(12)$ \\
\hline 18 & erectile & $273.8(28)$ & 68 & vellus & $32(7)$ \\
\hline 19 & boys & $253.2(7)$ & 69 & baldness & $30.5(14)$ \\
\hline 20 & hyperandrogenic & $242.4(7)$ & 70 & psa & $28.7(20)$ \\
\hline 21 & ejaculate & $238.1(7)$ & 71 & condom & $28.5(23)$ \\
\hline 22 & prostatitis & $233.2(13)$ & 72 & continence & $28(11)$ \\
\hline 23 & prostatectomies & $227.4(6)$ & 73 & vas & $28(11)$ \\
\hline 24 & bph & $209.4(25)$ & 74 & areata & $27(6)$ \\
\hline 25 & glans & $199.5(10)$ & 75 & levosimendan & $26.1(6)$ \\
\hline 26 & turp & $171.6(8)$ & 76 & coitus & $25.2(7)$ \\
\hline 27 & males & $170.8(30)$ & 77 & antiandrogen & $24.8(6)$ \\
\hline 28 & zp & $161.7(9)$ & 78 & semen & $24.7(15)$ \\
\hline 29 & prostate & $145.7(71)$ & 79 & osas & $24.1(6)$ \\
\hline 30 & deferens & $131.7(10)$ & 80 & flutamide & $23.7(6)$ \\
\hline 31 & periurethral & $126.8(9)$ & 81 & homosexual & $23.2(6)$ \\
\hline 32 & castration & $122.6(11)$ & 82 & androgens & 22.8 (19) \\
\hline 33 & spermatic & $111.6(6)$ & 83 & hirsutism & $22.2(9)$ \\
\hline 34 & intraurethral & $107.6(7)$ & 84 & male & $22.1 \quad(52)$ \\
\hline 35 & dihydrotestosterone & 105.6 & 85 & lhrh & $21.7(15)$ \\
\hline 36 & hypermobility & $102.2(10)$ & 86 & antiandrogens & $21.7(8)$ \\
\hline 37 & transurethral & $100.7(13)$ & 87 & laughing & $21.3(8)$ \\
\hline 38 & scrotal & $97.5(6)$ & 88 & ram & $21(9)$ \\
\hline 39 & dht & 96.4 (14) & 89 & clitoris & $20.6(6)$ \\
\hline 40 & corpora & $95.7(12)$ & 90 & intercourse & $20.6(15)$ \\
\hline 41 & sildenafil & $83.1(8)$ & 91 & insemination & $20(13)$ \\
\hline 42 & transrectal & $83(7)$ & 92 & guzmania & $19.6(8)$ \\
\hline 43 & urethra & $81.8(43)$ & 93 & testes & $19.6(11)$ \\
\hline 44 & spermatogenesis & 79.1 (11) & 94 & $\mathrm{dbh}$ & $19.5(5)$ \\
\hline 45 & impotency & $78.8(8)$ & 95 & zona & $19.2(8)$ \\
\hline 46 & finasteride & $75.1(6)$ & 96 & androgenic & $18.9(14)$ \\
\hline 47 & foreskin & 74.8 (7) & 97 & detrusor & $18.5(10)$ \\
\hline 48 & psma & $74.5(8)$ & 98 & infertile & $18.3(6)$ \\
\hline 49 & resectoscopes & $74.4(6)$ & 99 & voiding & $18.2(9)$ \\
\hline 50 & prostatic & $73.6(34)$ & 100 & gender & $18.1(10)$ \\
\hline
\end{tabular}


Table S-7: Percentage of patents classified by the authors as focusing on the needs of women and men, by MeSH sex tag

\begin{tabular}{rcccc}
\hline \hline & \multicolumn{3}{c}{ Human Review } \\
No Sex Tags & Not Sex Focused & Male Focused & Female Focused & Male \& Female Focused \\
\cline { 2 - 5 } Male Check Tag & 89 & 6 & 5 & 0 \\
Female Check Tag & 38 & 57 & 4 & 2 \\
Male \& Female Check Tag & 28 & 6 & 62 & 2 \\
\hline \hline
\end{tabular}


Table S-8: Patents tagged as female by the MTI address diseases with higher female incidence counts; male-tagged patents address diseases with higher male incidence

\begin{tabular}{|c|c|c|c|c|}
\hline \multirow{2}{*}{$\begin{array}{l}\text { Dependent Variables: } \\
\text { Model: }\end{array}$} & \multicolumn{2}{|c|}{ Log Female Incidence } & \multicolumn{2}{|c|}{ Log Male Incidence } \\
\hline & (1) & $(2)$ & $(3)$ & (4) \\
\hline \multicolumn{5}{|l|}{ Variables } \\
\hline Female MeSH & $\begin{array}{l}1.1^{* * *} \\
(0.02)\end{array}$ & $\begin{array}{l}1.3^{* * *} \\
(0.02)\end{array}$ & & $\begin{array}{c}-1.3^{* * *} \\
(0.02)\end{array}$ \\
\hline Male MeSH & & $\begin{array}{c}-0.69^{* * *} \\
(0.01)\end{array}$ & $\begin{array}{c}0.37^{* * *} \\
(0.01)\end{array}$ & $\begin{array}{c}0.73^{* * *} \\
(0.01)\end{array}$ \\
\hline Log Male Incidence & $\begin{array}{l}0.93^{* * *} \\
(0.001)\end{array}$ & $\begin{array}{l}0.93^{* * *} \\
(0.001)\end{array}$ & & \\
\hline Log Female Incidence & & & $\begin{array}{c}0.96^{* * *} \\
(0.0005)\end{array}$ & $\begin{array}{c}0.96^{* * *} \\
(0.0005)\end{array}$ \\
\hline (Intercept) & $\begin{array}{c}0.95^{* * *} \\
(0.01)\end{array}$ & $\begin{array}{c}1^{* * *} \\
(0.01)\end{array}$ & $\begin{array}{l}0.15^{* * *} \\
(0.006)\end{array}$ & $\begin{array}{l}0.27^{* * *} \\
(0.006)\end{array}$ \\
\hline \multicolumn{5}{|l|}{ Fit statistics } \\
\hline Observations & 662,727 & 662,727 & 662,727 & 662,727 \\
\hline $\mathrm{R}^{2}$ & 0.87311 & 0.87479 & 0.86924 & 0.87533 \\
\hline
\end{tabular}

Each observation is a patent-disease match.

Patents are inverse-weighted by the number of diseases each patent matches.

Standard-errors, clustered by patent, in parentheses.

Signif. Codes: ***: 0.001, **: 0.01, *: 0.05 
Table S-9: List of patents with our female-only trial prediction measure

\begin{tabular}{|c|c|c|c|}
\hline P(Female Trial) & P(Male Trial) & Patent title & Female Focused? \\
\hline $100 \%$ & $1 \%$ & Treatment of climacteric disorders with nitric oxide synthase substrates and/or donors & $\mathrm{Y}$ \\
\hline $99 \%$ & $0 \%$ & Transdermal hormone replacement therapy & $\mathrm{Y}$ \\
\hline $99 \%$ & $2 \%$ & Use of estradiol valerate or $17 E \leq \leq$ estradiol in combination with ... increase feminine libido & $\mathrm{Y}$ \\
\hline $98 \%$ & $0 \%$ & Benzothiophene compounds, compositions, and methods & $\mathrm{Y}$ \\
\hline $95 \%$ & $3 \%$ & Connection mechanisms for uterine mobilizer & $\mathrm{Y}$ \\
\hline $90 \%$ & $0 \%$ & Detection of group B Streptococcus & $\mathrm{Y}$ \\
\hline $88 \%$ & $2 \%$ & Prostaglandine derivatives & \\
\hline $88 \%$ & $0 \%$ & Nursing apparatus & $\mathrm{Y}$ \\
\hline $85 \%$ & $5 \%$ & Treatment of cellulite and adipose tissue with mid-infrared radiation & $\mathrm{Y}$ \\
\hline $85 \%$ & $1 \%$ & Catheter for measuring intrauterine pressure & $\mathrm{Y}$ \\
\hline $79 \%$ & $0 \%$ & Urinary incontinence device & \\
\hline $77 \%$ & $5 \%$ & Sanitary feminine napkin with a mechanism for optional contouring & $\mathrm{Y}$ \\
\hline $74 \%$ & $7 \%$ & Method for increasing placental blood flow & $\mathrm{Y}$ \\
\hline $74 \%$ & $0 \%$ & Indenone derivative and pharmaceutical composition comprising same & $\mathrm{Y}$ \\
\hline $73 \%$ & $0 \%$ & Apparatus for expressing milk & $\mathrm{Y}$ \\
\hline $67 \%$ & $4 \%$ & Method for isolating a novel receptor for alpha.4 integrins & \\
\hline $63 \%$ & $6 \%$ & Absorbent article having tucked flaps & $\mathrm{Y}$ \\
\hline $63 \%$ & $0 \%$ & Ultrasound device for use in a thermotherapy apparatus & $\mathrm{Y}$ \\
\hline $62 \%$ & $0 \%$ & Liquid handling member with inner materials having good creep... & \\
\hline $61 \%$ & $2 \%$ & Tetrahydrocarbazole derivatives and their pharmaceutical use & $\mathrm{Y}$ \\
\hline $58 \%$ & $0 \%$ & Breathing circuit connector for use in anesthesiology & \\
\hline $55 \%$ & $3 \%$ & Methods of using growth inhibitory peptides & \\
\hline $55 \%$ & $1 \%$ & Absorbent products having integral transverse retaining tab and pocket & $\mathrm{Y}$ \\
\hline $54 \%$ & $3 \%$ & Breastpump with stimulation and method & $\mathrm{Y}$ \\
\hline $53 \%$ & $0 \%$ & Anti-midkine antibody for preventing post-laparotomy adhesions & \\
\hline $50 \%$ & $4 \%$ & Angiostatin fragments and method of use & \\
\hline $49 \%$ & $17 \%$ & Apparatus and method for displaying three-dimensional image and ultrasonic ... & \\
\hline $46 \%$ & $31 \%$ & Method for preparation of androst-4-ene-3,17-dione and androsta-1,4-diene-3,17-dione & \\
\hline $44 \%$ & $1 \%$ & Static devices and methods to shrink tissues for incontinence & Y \\
\hline $41 \%$ & $7 \%$ & Pharmaceutically active compounds and methods of use & \\
\hline $40 \%$ & $2 \%$ & Apparatus and process for monitoring a pregnant mammal & \\
\hline $38 \%$ & $0 \%$ & Tissue expander stent & $\mathrm{Y}$ \\
\hline $35 \%$ & $2 \%$ & Equal phase two-dimensional array probe & \\
\hline $33 \%$ & $14 \%$ & Method and portable device for treating skin disorders & \\
\hline $33 \%$ & $3 \%$ & Device for the height-adjustable fixing and support of internal anatomical organs & \\
\hline $29 \%$ & $7 \%$ & Treatment of cardiac dysfunction with 15-ketoprostaglandin compounds & \\
\hline $24 \%$ & $5 \%$ & Liquid absorbing material and method for making the same & $\mathrm{Y}$ \\
\hline $23 \%$ & $0 \%$ & Soybean cultivar 9380279522807 & \\
\hline $23 \%$ & $6 \%$ & Cosmetic agent on the basis of quaternary chitosan derivatives, novel quaternary... & \\
\hline $21 \%$ & $3 \%$ & Power transmission apparatus for electric bending endoscope & \\
\hline $16 \%$ & $0 \%$ & Culture flask & \\
\hline $12 \%$ & $1 \%$ & Method for enhancing functional properties of submucosal tissue graft constructs & \\
\hline $12 \%$ & $7 \%$ & Method and apparatus for visualization of motion in ultrasound flow imaging ... & \\
\hline $11 \%$ & $32 \%$ & Method for producing an L-amino acid using a bacterium of the Enterobacteriaceae ... & \\
\hline $11 \%$ & $5 \%$ & Delivery of nucleic acid to cells & \\
\hline $3 \%$ & $6 \%$ & Glass-like polysaccharide useful as absorbent for liquids & \\
\hline $2 \%$ & $18 \%$ & Controlled-release formulations, method of manufacture, and use thereof & \\
\hline $2 \%$ & $2 \%$ & Therapeutic catheter with displacement sensing transducer & \\
\hline $1 \%$ & $0 \%$ & Versatile operatory light system & \\
\hline $0 \%$ & $0 \%$ & Defibrillation apparatus and method & \\
\hline
\end{tabular}


Table S-10: List of patents with our male-only trial prediction measure

\begin{tabular}{|c|c|c|c|}
\hline P(Female Trial) & P(Male Trial) & Patent title & Male Focused? \\
\hline $0 \%$ & $100 \%$ & Certain phosphonomethyl-pentanedioic acid derivatives thereof & $\mathrm{Y}$ \\
\hline $2 \%$ & $99 \%$ & Method for treatment of alopecia & $\mathrm{Y}$ \\
\hline $1 \%$ & $99 \%$ & Implantable penile prosthetic cylinder with inclusive fluid reservoir & $\mathrm{Y}$ \\
\hline $0 \%$ & $98 \%$ & Alpha interferon for treating prostate cancer & $\mathrm{Y}$ \\
\hline $2 \%$ & $95 \%$ & Benzimidazole derivatives useful as selective androgen receptor modulators (sarms) & $\mathrm{Y}$ \\
\hline $49 \%$ & $85 \%$ & 17-CE $\leq$ hydroxysteroid dehydrogenase type 3 inhibitors for the treatment of androgen... & $\mathrm{Y}$ \\
\hline $0 \%$ & $85 \%$ & Azolyl substituted aralkyl compounds and pesticidal use thereof & \\
\hline $2 \%$ & $84 \%$ & Purified luciferase from luciola lateralis & \\
\hline $5 \%$ & $82 \%$ & Cosmetic or pharmaceutical composition for topical use to prevent ....androgenetic alopecia & $\mathrm{Y}$ \\
\hline $1 \%$ & $82 \%$ & Implantable penile prosthesis & $\mathrm{Y}$ \\
\hline $0 \%$ & $79 \%$ & Herbal compositions for prostate conditions & $\mathrm{Y}$ \\
\hline $2 \%$ & $78 \%$ & Benzonaphthyridines & \\
\hline $0 \%$ & $75 \%$ & Method of determining the presence of endotoxin in a sample & \\
\hline $0 \%$ & $72 \%$ & $N$-phenoxypropanol-N'-pyridazinyl ethylendiamines as beta.-receptor blockers & $\mathrm{Y}$ \\
\hline $4 \%$ & $72 \%$ & Condensed polycyclic compounds & $\mathrm{Y}$ \\
\hline $1 \%$ & $70 \%$ & 1(p-Chlorobenzoyl)-5-methoxy-2-methyl indole-3-acetic acid-3-oxo-isobenzofuranyl... & \\
\hline $3 \%$ & $64 \%$ & Stable colored reference standard for enzymatic determinations & \\
\hline $0 \%$ & $64 \%$ & Treatment and diagnosis of cancer & $\mathrm{Y}$ \\
\hline $0 \%$ & $63 \%$ & Anti-hypertensive polyhaloisopropyl-substituted arylureas & $\mathrm{Y}$ \\
\hline $0 \%$ & $63 \%$ & 3-alkoxy-1-phenylpyrazole derivatives and pesticides & \\
\hline $16 \%$ & $60 \%$ & Photostable cosmetic light screening compositions & \\
\hline $1 \%$ & $59 \%$ & Methods of using soluble epoxide hydrolase inhibitors & \\
\hline $5 \%$ & $57 \%$ & Methods of using 2,4-dienoic acid esters of tocopherols to reduce free radical damage... & \\
\hline $1 \%$ & $52 \%$ & Method of treating helminthiasis by parenteral administration of sulfide- and .... & $\mathrm{Y}$ \\
\hline $1 \%$ & $52 \%$ & Analogues of the active metabolite of leflunomide & \\
\hline $0 \%$ & $49 \%$ & Hair conditioner compositions having improved freezing and freeze-thaw stability & \\
\hline $0 \%$ & $47 \%$ & Novel compounds & \\
\hline $1 \%$ & $47 \%$ & Composition and method to modify sperm function and increase male gender ratio ... & $\mathrm{Y}$ \\
\hline $2 \%$ & $45 \%$ & Novel HMG-COA reductase inhibitors & $\mathrm{Y}$ \\
\hline $0 \%$ & $41 \%$ & 3-Substituted-5-alkyl-1,6-naphthyridin-2(1H)-ones, cardiotonic use and preparation... & \\
\hline $2 \%$ & $38 \%$ & Microbiological process for oxidation of methyl groups & \\
\hline $5 \%$ & $38 \%$ & Lactamimides as calcium antagonists & \\
\hline $26 \%$ & $34 \%$ & Osmotic drug delivery monitoring system and method & \\
\hline $1 \%$ & $33 \%$ & Glucose dehydrogenase and its production & \\
\hline $3 \%$ & $31 \%$ & Method for the oxidation of aromatic compounds & \\
\hline $3 \%$ & $27 \%$ & Method for detection of long-lived radioisotopes in small biochemical samples & \\
\hline $4 \%$ & $27 \%$ & Benzocycloheptapyrans, compositions, and method of treatment & \\
\hline $3 \%$ & $25 \%$ & Benzoindoline compounds & \\
\hline $1 \%$ & $25 \%$ & Family GH 61 polypeptides & \\
\hline $1 \%$ & $22 \%$ & Pyridopyrimidinone antianginal agents & \\
\hline $3 \%$ & $20 \%$ & Water-degradable fishing lure & \\
\hline $3 \%$ & $19 \%$ & Quinoline derivatives 057 & \\
\hline $7 \%$ & $15 \%$ & Flavonoid complexes with cyclodextrins & \\
\hline $0 \%$ & $12 \%$ & Triazine derivatives, and pharmaceutical compositions comprising the same & \\
\hline $9 \%$ & $11 \%$ & Ultrasonic diagnostic apparatus capable of performing switching between single beam ... & \\
\hline $21 \%$ & $5 \%$ & Method of producing products for use in the treatment of bacterial and/or virus infections & \\
\hline $0 \%$ & $5 \%$ & Quinoxalinones as antibacterial composition & \\
\hline $5 \%$ & $4 \%$ & Multi-chamber vial & \\
\hline $5 \%$ & $2 \%$ & Method and device for applying external pressure in combination with ... & \\
\hline $1 \%$ & $0 \%$ & Recessed tip fluid dispenser & \\
\hline
\end{tabular}




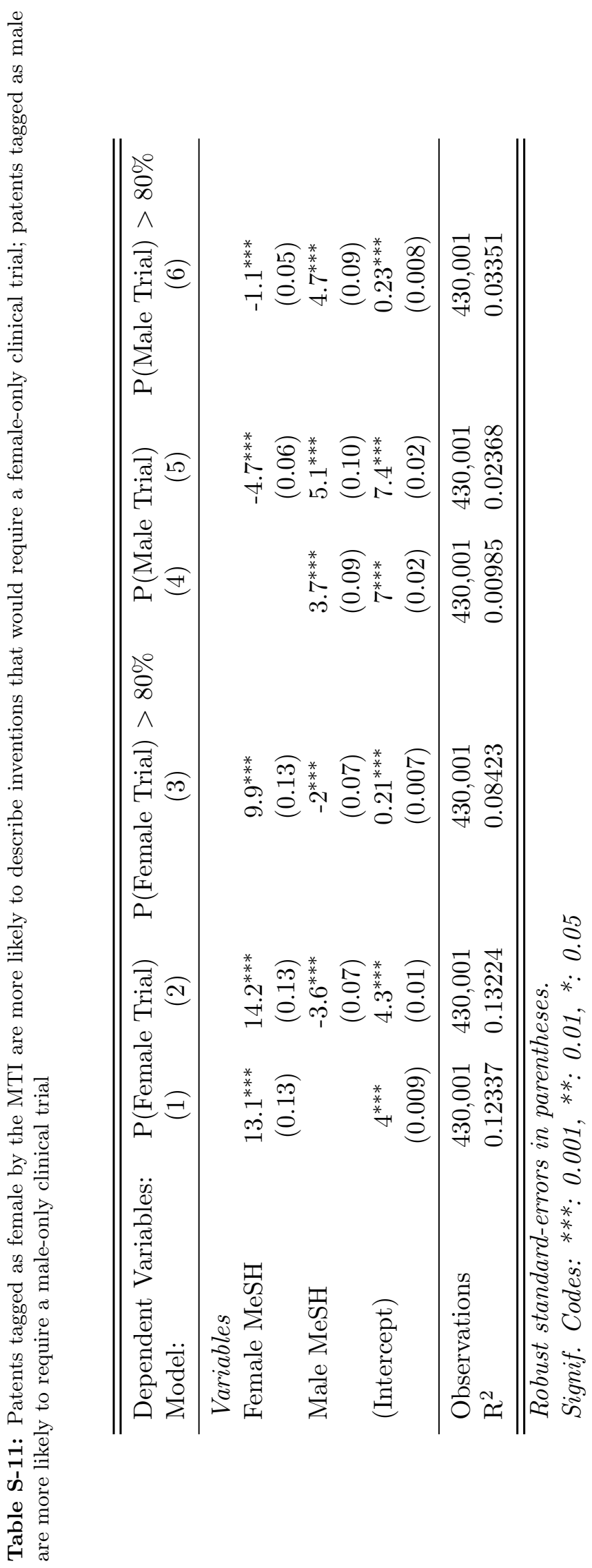


Table S-12: Gender-identification success rates, by team size and geographic location

\begin{tabular}{rrr}
\hline \hline Inventor count & Non-US teams & US teams \\
\hline 1 & 0.800 & 0.908 \\
2 & 0.690 & 0.819 \\
3 & 0.605 & 0.744 \\
4 & 0.539 & 0.677 \\
5 & 0.491 & 0.615 \\
6 & 0.460 & 0.559 \\
7 & 0.426 & 0.495 \\
8 & 0.371 & 0.468 \\
9 & 0.324 & 0.467 \\
10 & 0.279 & 0.398 \\
$>10$ & 0.308 & 0.387 \\
\hline \hline
\end{tabular}




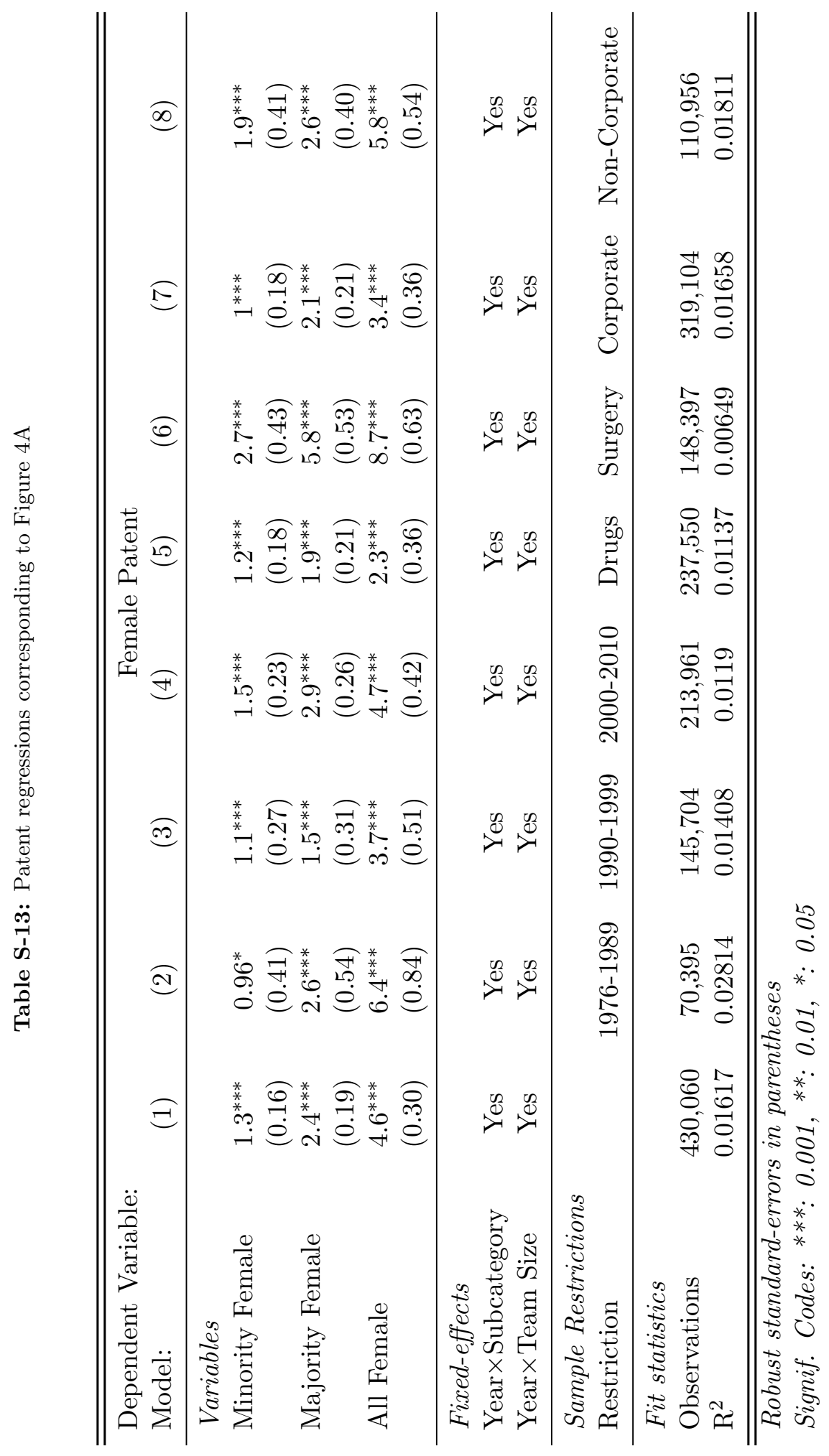




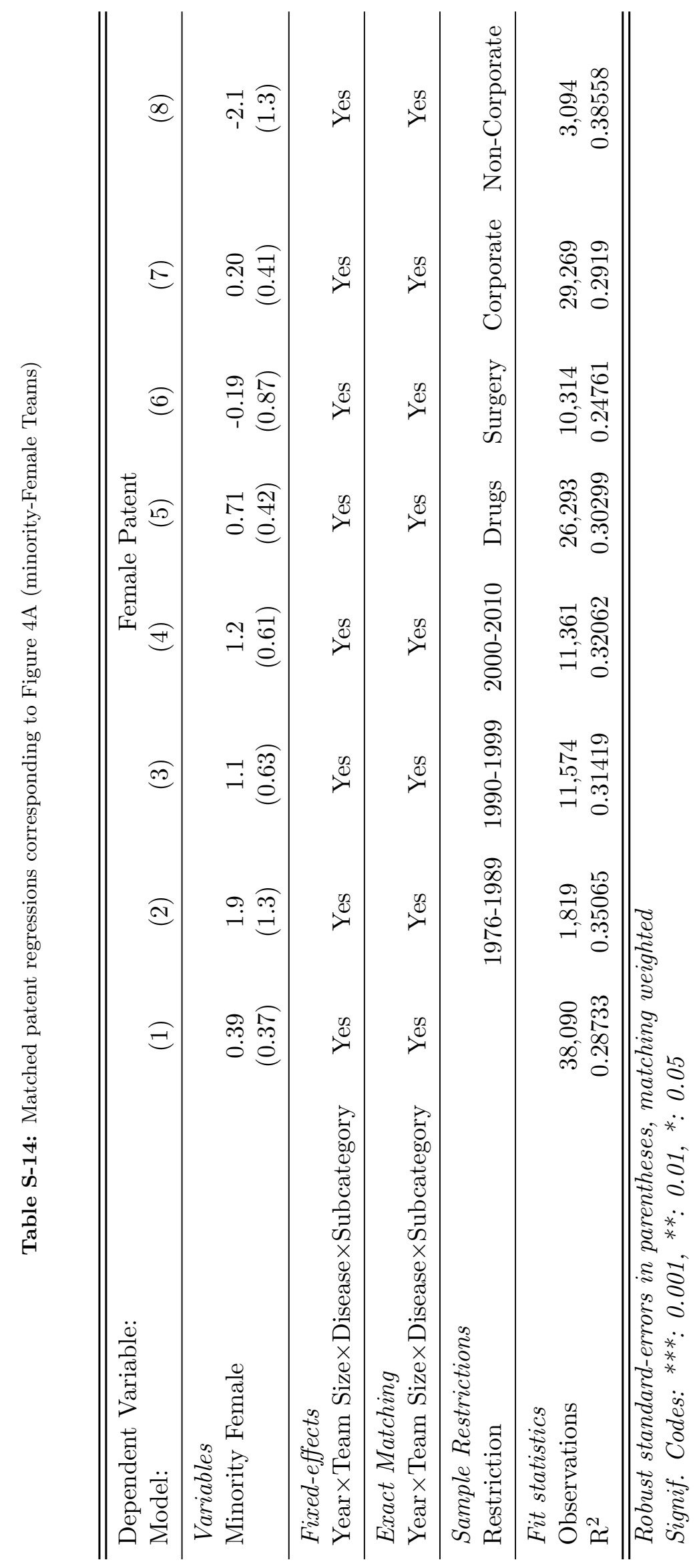




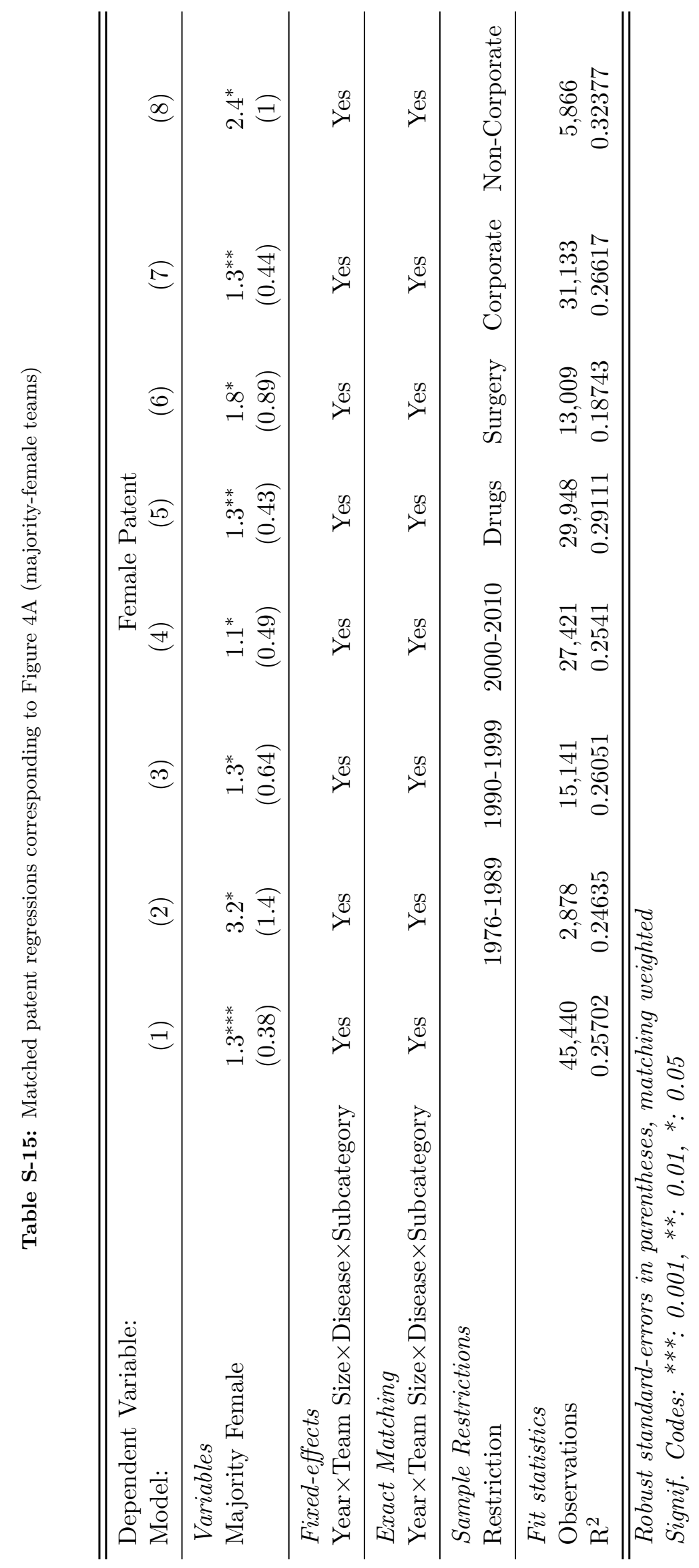




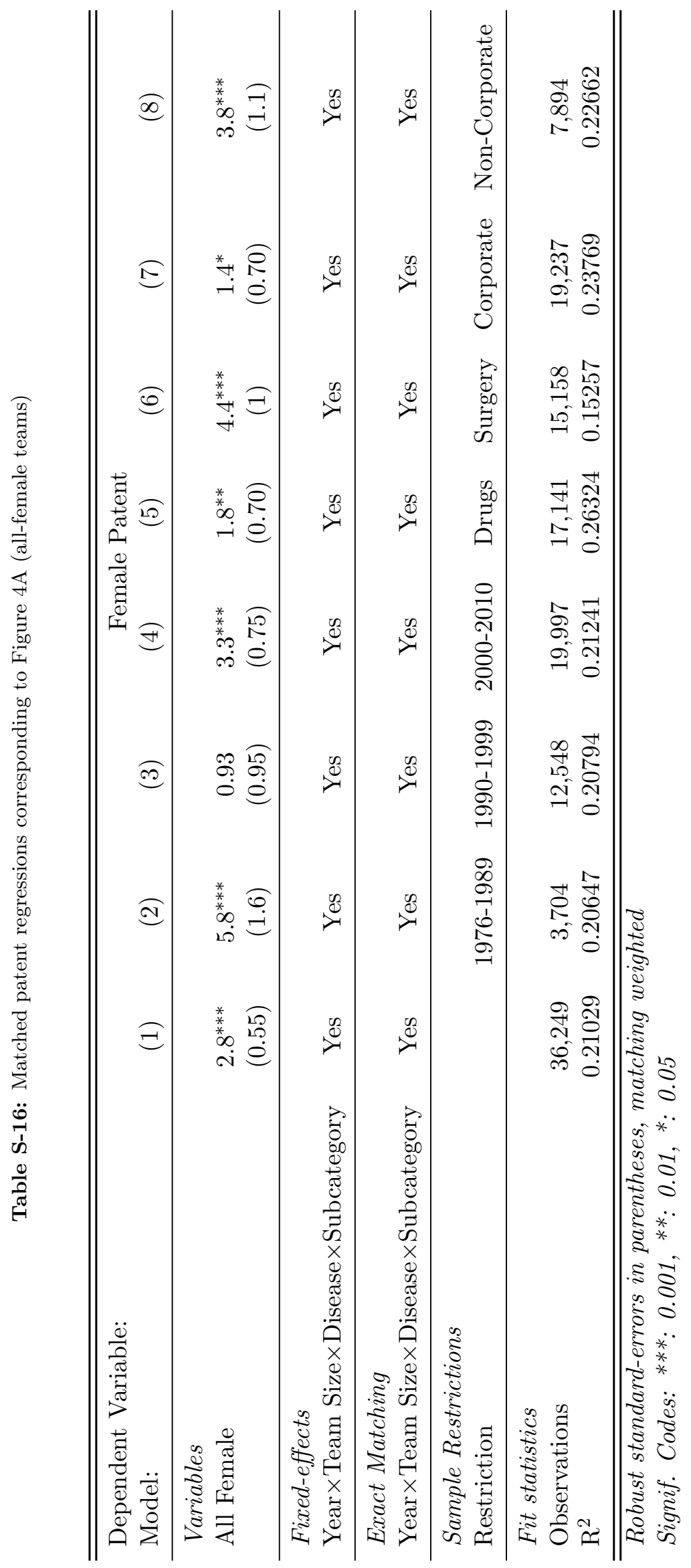




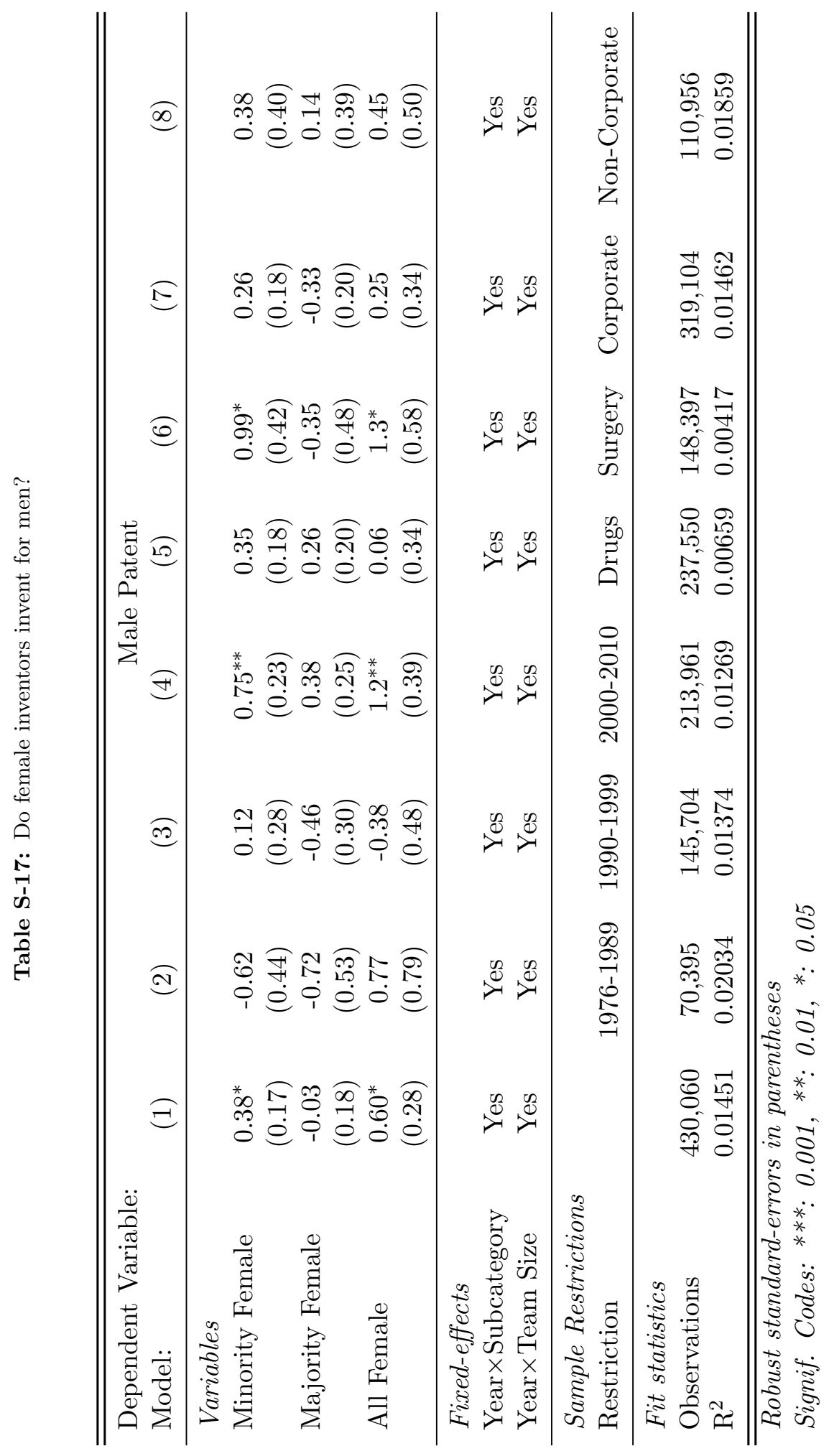




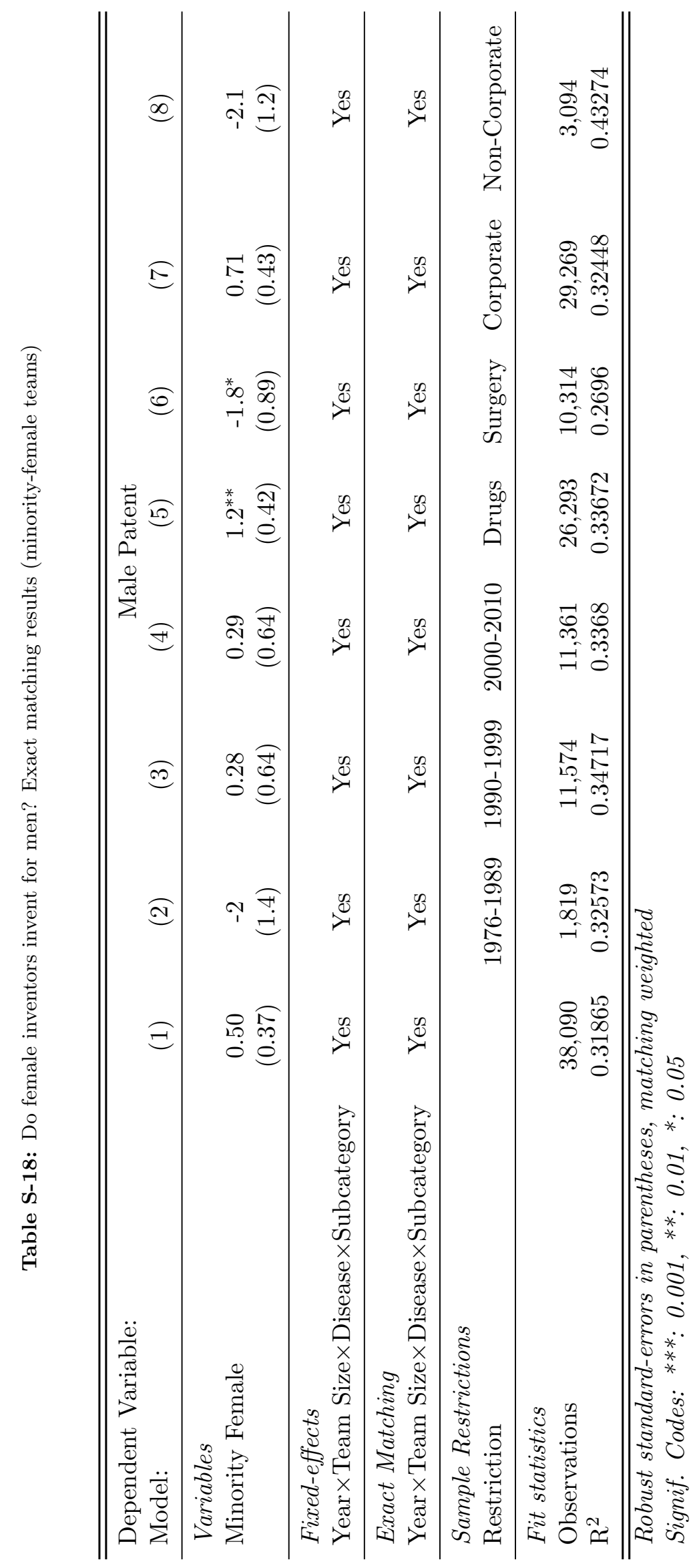




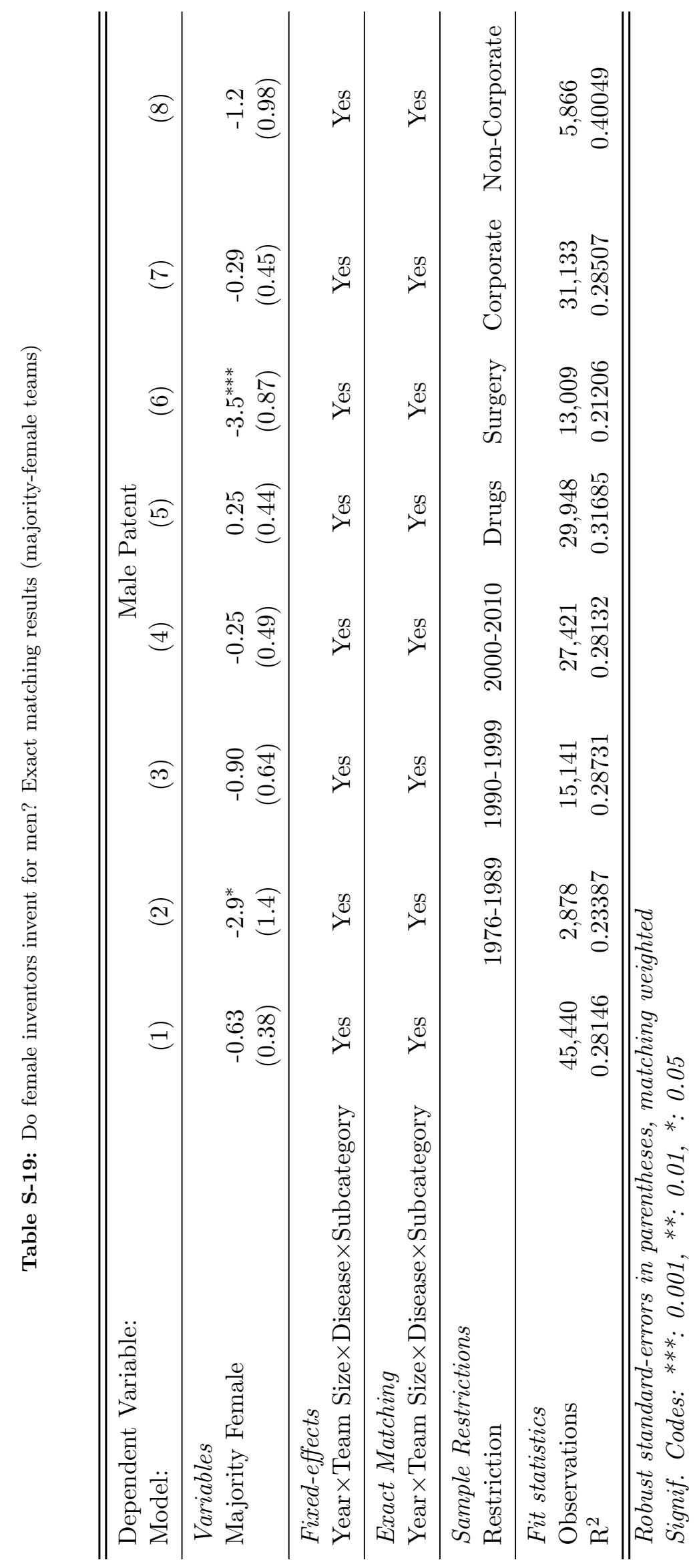




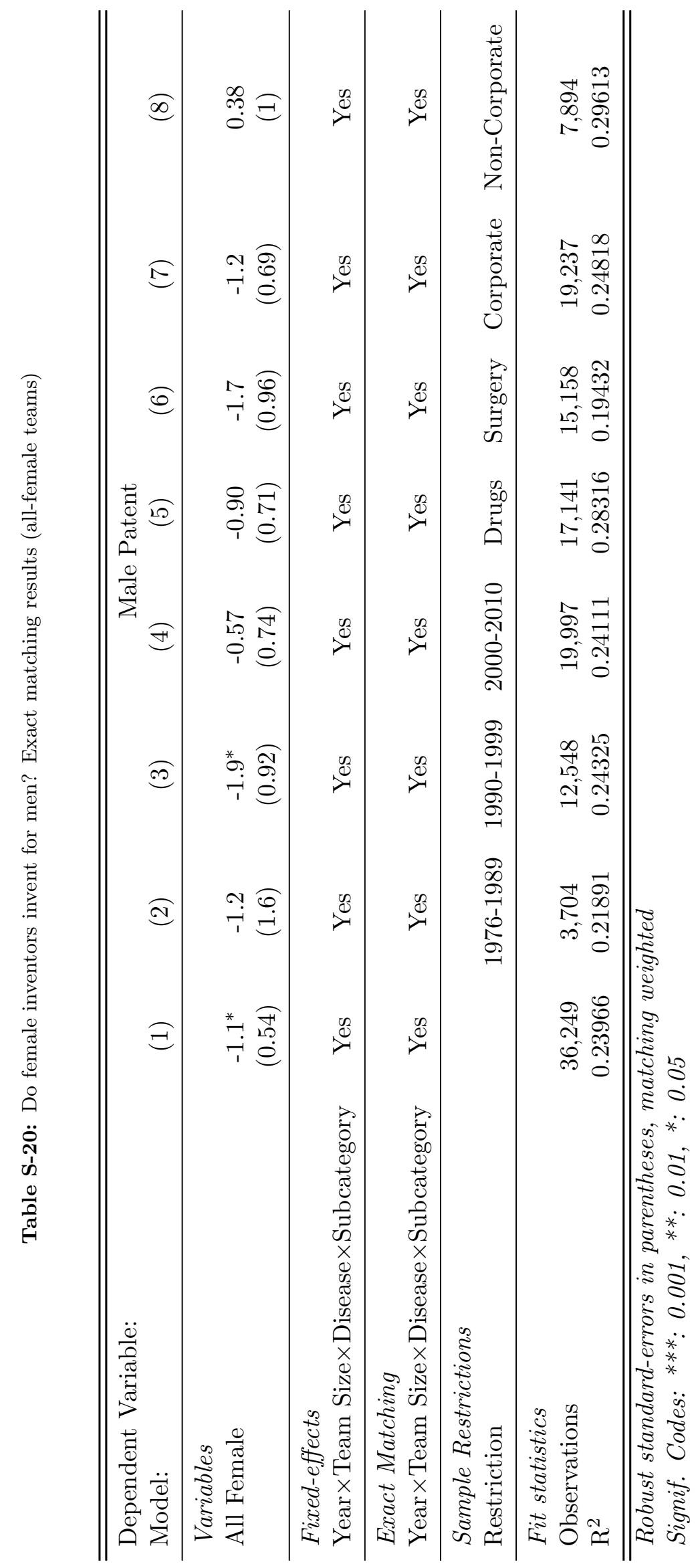




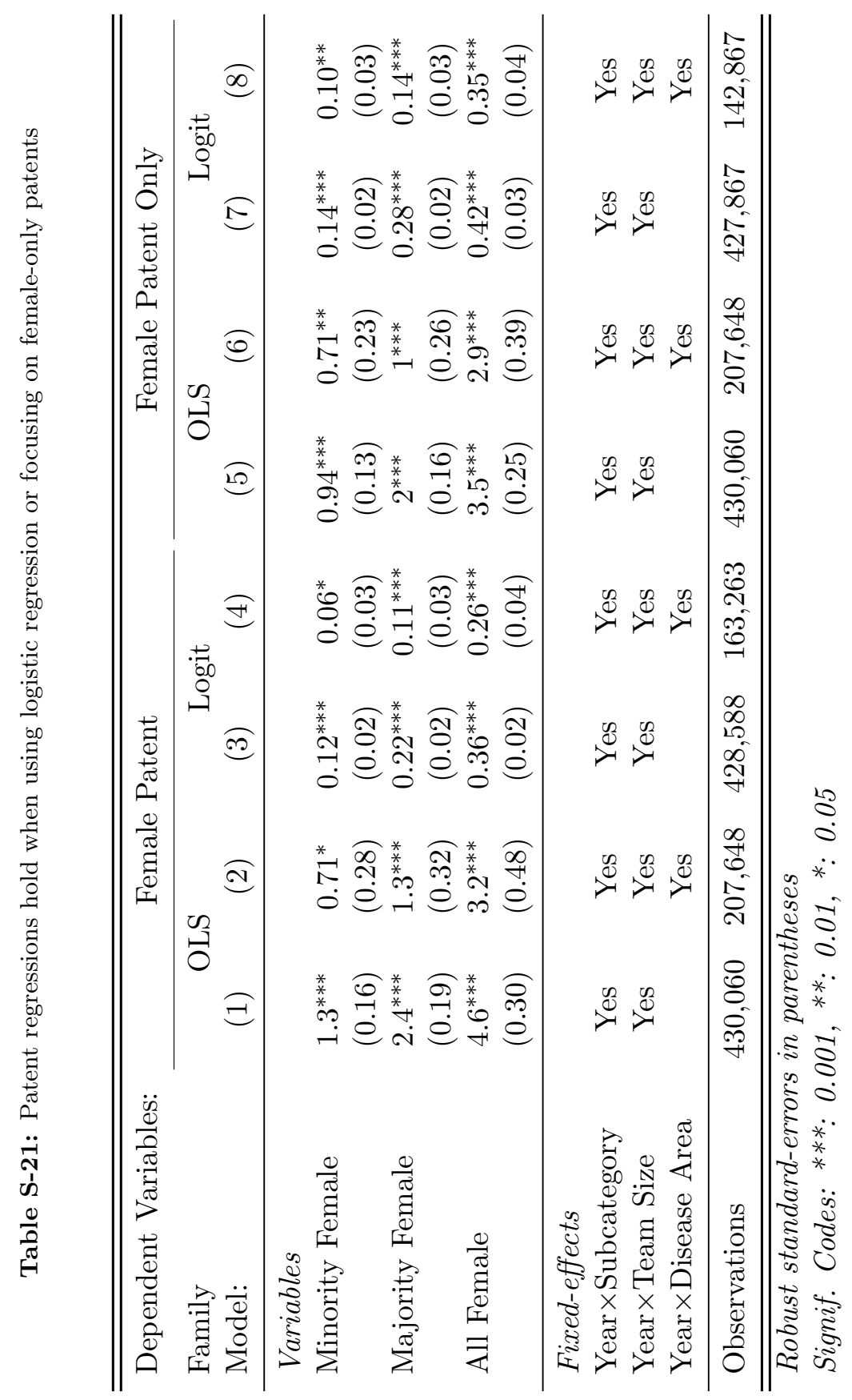


Table S-22: Patent regressions hold when we feed more or less patent text to the MTI algorithm

\begin{tabular}{|c|c|c|c|c|c|c|c|c|}
\hline \multirow{2}{*}{$\begin{array}{l}\text { Dependent Variable: } \\
\text { Model: }\end{array}$} & \multicolumn{8}{|c|}{ Female Patent } \\
\hline & \multicolumn{2}{|c|}{ 2,500 Characters } & \multicolumn{2}{|c|}{ Title and Abstract } & \multicolumn{2}{|c|}{ Strict Filtering } & \multicolumn{2}{|c|}{ 10,000 Characters } \\
\hline \multicolumn{9}{|l|}{ Variables } \\
\hline Minority Female & $\begin{array}{l}1.3^{* * *} \\
(0.16)\end{array}$ & $\begin{array}{l}0.71^{*} \\
(0.28)\end{array}$ & $\begin{array}{l}1.2^{* * *} \\
(0.15)\end{array}$ & $\begin{array}{c}1.00^{* * *} \\
(0.28)\end{array}$ & $\begin{array}{c}0.67^{* * *} \\
(0.13)\end{array}$ & $\begin{array}{c}0.38 \\
(0.21)\end{array}$ & $\begin{array}{l}1.6^{* * *} \\
(0.19)\end{array}$ & $\begin{array}{l}0.81^{*} \\
(0.35)\end{array}$ \\
\hline Majority Female & $\begin{array}{l}2.4^{* * *} \\
(0.19)\end{array}$ & $\begin{array}{l}1.3^{* * *} \\
(0.32)\end{array}$ & $\begin{array}{l}2.1^{* * *} \\
(0.17)\end{array}$ & $\begin{array}{l}1.5^{* * *} \\
(0.31)\end{array}$ & $\begin{array}{l}1.5^{* * *} \\
(0.15)\end{array}$ & $\begin{array}{l}0.62^{* *} \\
(0.24)\end{array}$ & $\begin{array}{l}2.7^{* * *} \\
(0.21)\end{array}$ & $\begin{array}{l}1.5^{* * *} \\
(0.38)\end{array}$ \\
\hline All Female & $\begin{array}{l}4.6^{* * *} \\
(0.30)\end{array}$ & $\begin{array}{l}3.2^{* * *} \\
(0.48)\end{array}$ & $\begin{array}{l}3.8^{* * *} \\
(0.28)\end{array}$ & $\begin{array}{l}3.1^{* * *} \\
(0.47)\end{array}$ & $\begin{array}{l}3.1^{* * *} \\
(0.25)\end{array}$ & $\begin{array}{l}2.4^{* * *} \\
(0.38)\end{array}$ & $\begin{array}{l}5.1^{* * *} \\
(0.33)\end{array}$ & $\begin{array}{l}4.3^{* * *} \\
(0.56)\end{array}$ \\
\hline \multicolumn{9}{|l|}{ Fixed-effects } \\
\hline Year $\times$ Subcategory & Yes & Yes & Yes & Yes & Yes & Yes & Yes & Yes \\
\hline Year $\times$ Team Size & Yes & Yes & Yes & Yes & Yes & Yes & Yes & Yes \\
\hline Year $\times$ Disease Area & & Yes & & Yes & & Yes & & Yes \\
\hline \multicolumn{9}{|l|}{ Fit statistics } \\
\hline Observations & 430,060 & 207,648 & 430,117 & 203,284 & 430,107 & 211,366 & 430,102 & 203,285 \\
\hline $\mathrm{R}^{2}$ & 0.01617 & 0.2878 & 0.01405 & 0.27091 & 0.01049 & 0.30259 & 0.01575 & 0.26548 \\
\hline
\end{tabular}

Robust standard-errors in parentheses

Signif. Codes: ***: 0.001, **: 0.01, *: 0.05 
Table S-23: Patent regressions hold when we restrict our sample to patents tagged as focusing on "humans" or by excluding patents tagged as focusing on "animals"

\begin{tabular}{|c|c|c|c|c|}
\hline $\begin{array}{l}\text { Dependent Variable: } \\
\text { Model: }\end{array}$ & $(1)$ & $(2)$ & $\begin{array}{c}\text { male Patent } \\
\qquad(3)\end{array}$ & $(4)$ \\
\hline \multicolumn{5}{|l|}{ Variables } \\
\hline Minority Female & $\begin{array}{l}1.5^{* * *} \\
(0.23)\end{array}$ & $\begin{array}{l}0.69^{*} \\
(0.34)\end{array}$ & $\begin{array}{l}1.3^{* * *} \\
(0.19)\end{array}$ & $\begin{array}{c}0.77^{*} \\
(0.34)\end{array}$ \\
\hline Majority Female & $\begin{array}{l}2.9^{* * *} \\
(0.26)\end{array}$ & $\begin{array}{l}1.5^{\text {*** }} \\
(0.38)\end{array}$ & $\begin{array}{l}2.6^{* * *} \\
(0.22)\end{array}$ & $\begin{array}{l}1.1^{* *} \\
(0.39)\end{array}$ \\
\hline All Female & $\begin{array}{l}5.3^{* * *} \\
(0.39)\end{array}$ & $\begin{array}{l}3.4^{* * *} \\
(0.57)\end{array}$ & $\begin{array}{l}5.1^{* * *} \\
(0.35)\end{array}$ & $\begin{array}{l}3.4^{* * *} \\
(0.56)\end{array}$ \\
\hline MeSH Sample & "Humans" & "Humans" & Not "Animals" & Not "Animals" \\
\hline $\begin{array}{l}\text { Fixed-effects } \\
\text { Year } \times \text { Subcategory } \\
\text { Year } \times \text { Team Size } \\
\text { Year } \times \text { Disease Area }\end{array}$ & $\begin{array}{l}\text { Yes } \\
\text { Yes }\end{array}$ & $\begin{array}{l}\text { Yes } \\
\text { Yes } \\
\text { Yes }\end{array}$ & $\begin{array}{l}\text { Yes } \\
\text { Yes }\end{array}$ & $\begin{array}{l}\text { Yes } \\
\text { Yes } \\
\text { Yes }\end{array}$ \\
\hline $\begin{array}{l}\text { Fit statistics } \\
\text { Observations } \\
\mathrm{R}^{2}\end{array}$ & $\begin{array}{l}303,962 \\
0.00695\end{array}$ & $\begin{array}{l}169,610 \\
0.29313\end{array}$ & $\begin{array}{l}336,150 \\
0.02006\end{array}$ & $\begin{array}{l}158,542 \\
0.29734\end{array}$ \\
\hline
\end{tabular}

Robust standard-errors in parentheses

Signif. Codes: ***: 0.001, **: 0.01, *: 0.05 


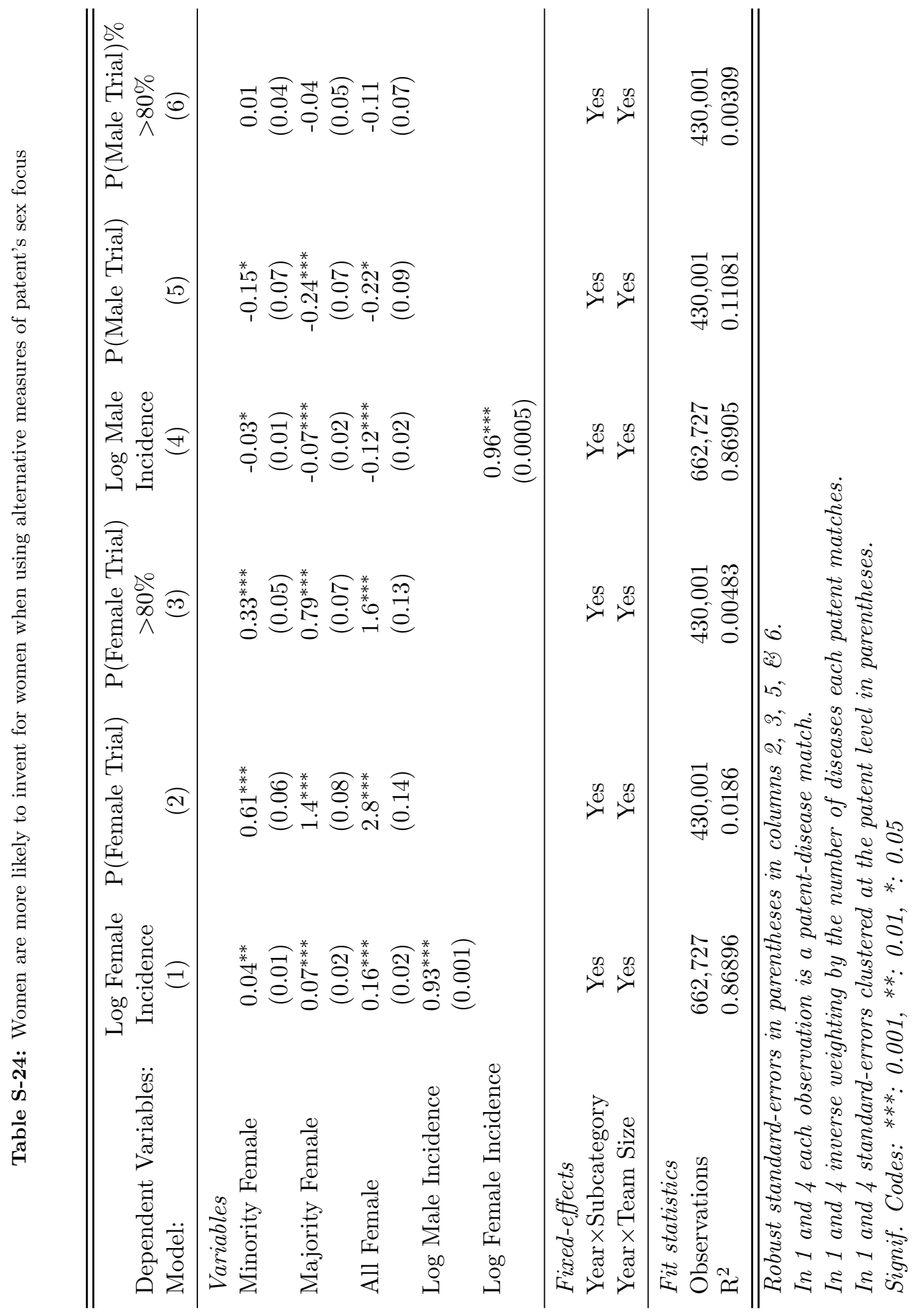




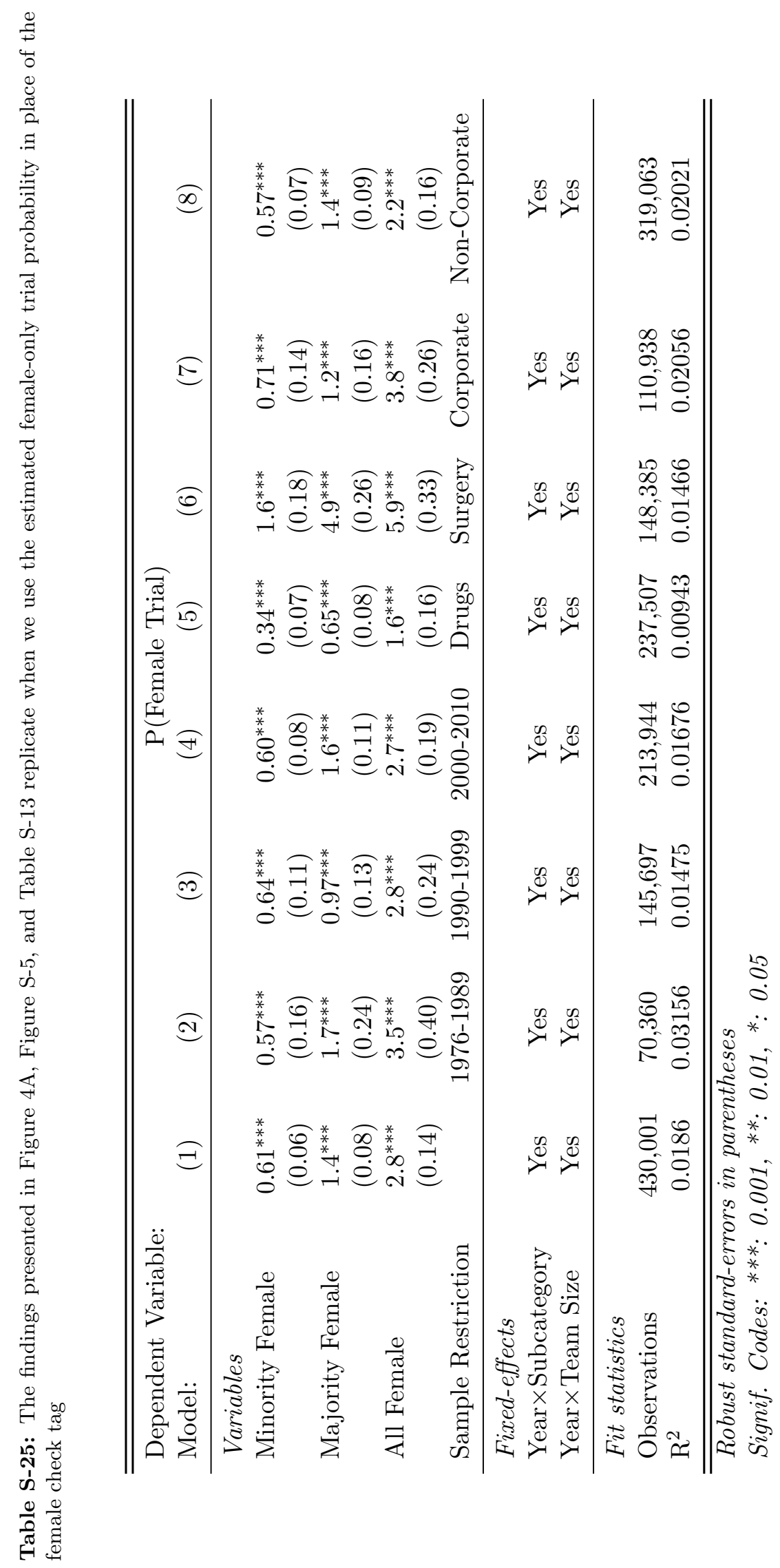




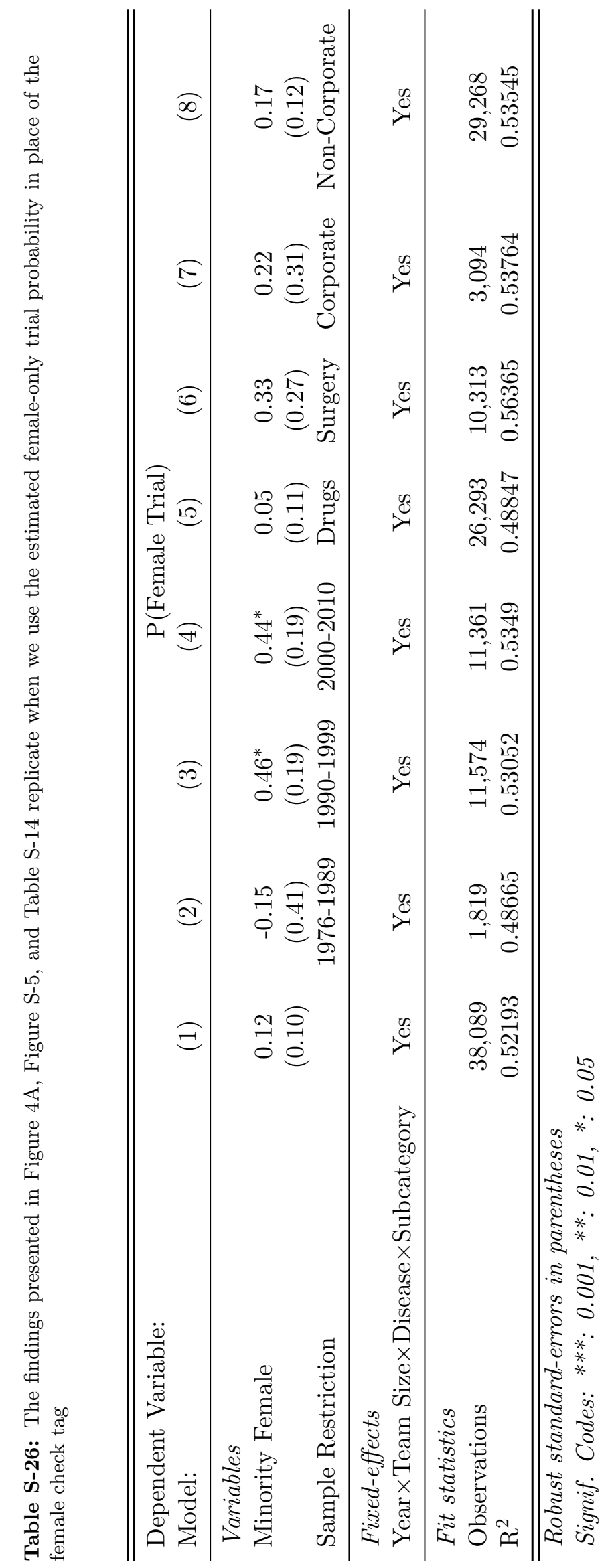




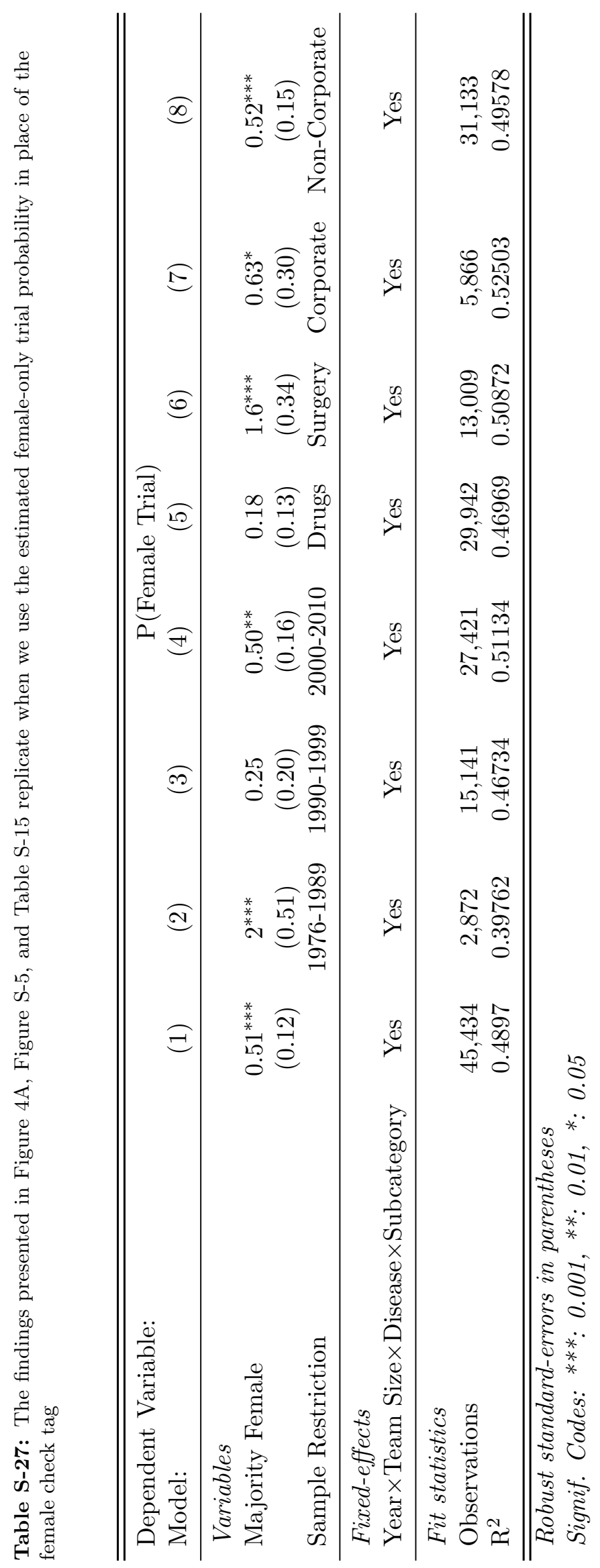




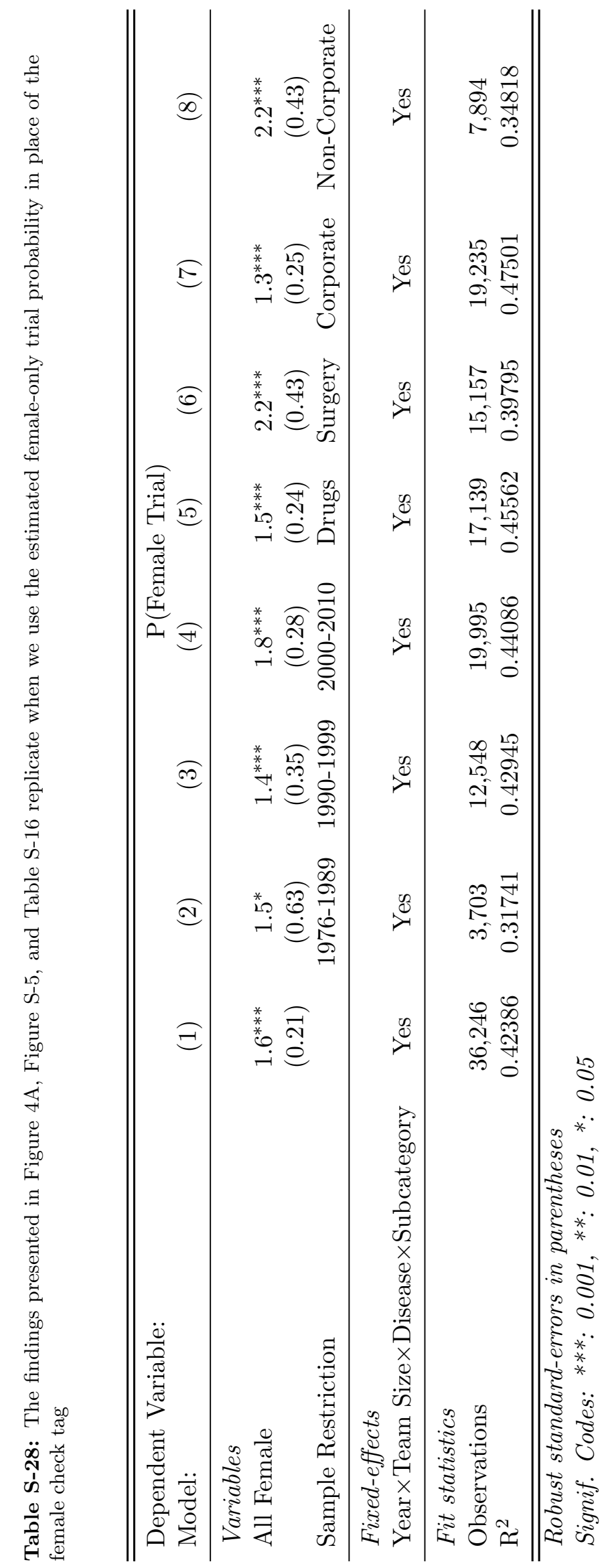


Table S-29: The findings hold when we match on Disease-Subcategory and Year-Disease-Subcategory

\begin{tabular}{|c|c|c|c|c|c|c|}
\hline $\begin{array}{l}\text { Dependent Variable: } \\
\text { Model: }\end{array}$ & (1) & $(2)$ & $\begin{array}{l}\text { Female } \\
(3)\end{array}$ & $\begin{array}{l}\text { Patent } \\
\qquad(4)\end{array}$ & $(5)$ & (6) \\
\hline \multicolumn{7}{|l|}{ Variables } \\
\hline Minority Female & $\begin{array}{l}1.3^{* * *} \\
(0.28)\end{array}$ & $\begin{array}{l}1.1^{* * *} \\
(0.31)\end{array}$ & & & & \\
\hline Majority Female & & & $\begin{array}{l}1.4^{* * *} \\
(0.30)\end{array}$ & $\begin{array}{l}1.4^{* * *} \\
(0.33)\end{array}$ & & \\
\hline All Female & & & & & $\begin{array}{l}2.5^{* * *} \\
(0.45)\end{array}$ & $\begin{array}{l}2.6^{* * *} \\
(0.50)\end{array}$ \\
\hline \multicolumn{7}{|l|}{ Fixed-effects } \\
\hline Disease $\times$ Subcategory & Yes & Yes & Yes & Yes & Yes & Yes \\
\hline Year $\times$ Disease $\times$ Subcategory & & Yes & & Yes & & Yes \\
\hline Year $\times$ Team Size & Yes & Yes & Yes & Yes & Yes & Yes \\
\hline \multicolumn{7}{|l|}{ Exact Matching } \\
\hline Disease $\times$ Subcategory & Yes & & Yes & & Yes & \\
\hline Year $\times$ Disease $\times$ Subcategory & & Yes & & Yes & & Yes \\
\hline \multicolumn{7}{|l|}{ Fit statistics } \\
\hline Observations & 170,853 & 121,495 & 157,009 & 100,896 & 140,208 & 71,180 \\
\hline $\mathrm{R}^{2}$ & 0.15909 & 0.20243 & 0.16027 & 0.19412 & 0.15213 & 0.18252 \\
\hline
\end{tabular}

Robust standard-errors in parentheses

Signif. Codes: ***: 0.001, **: 0.01, *: 0.05 


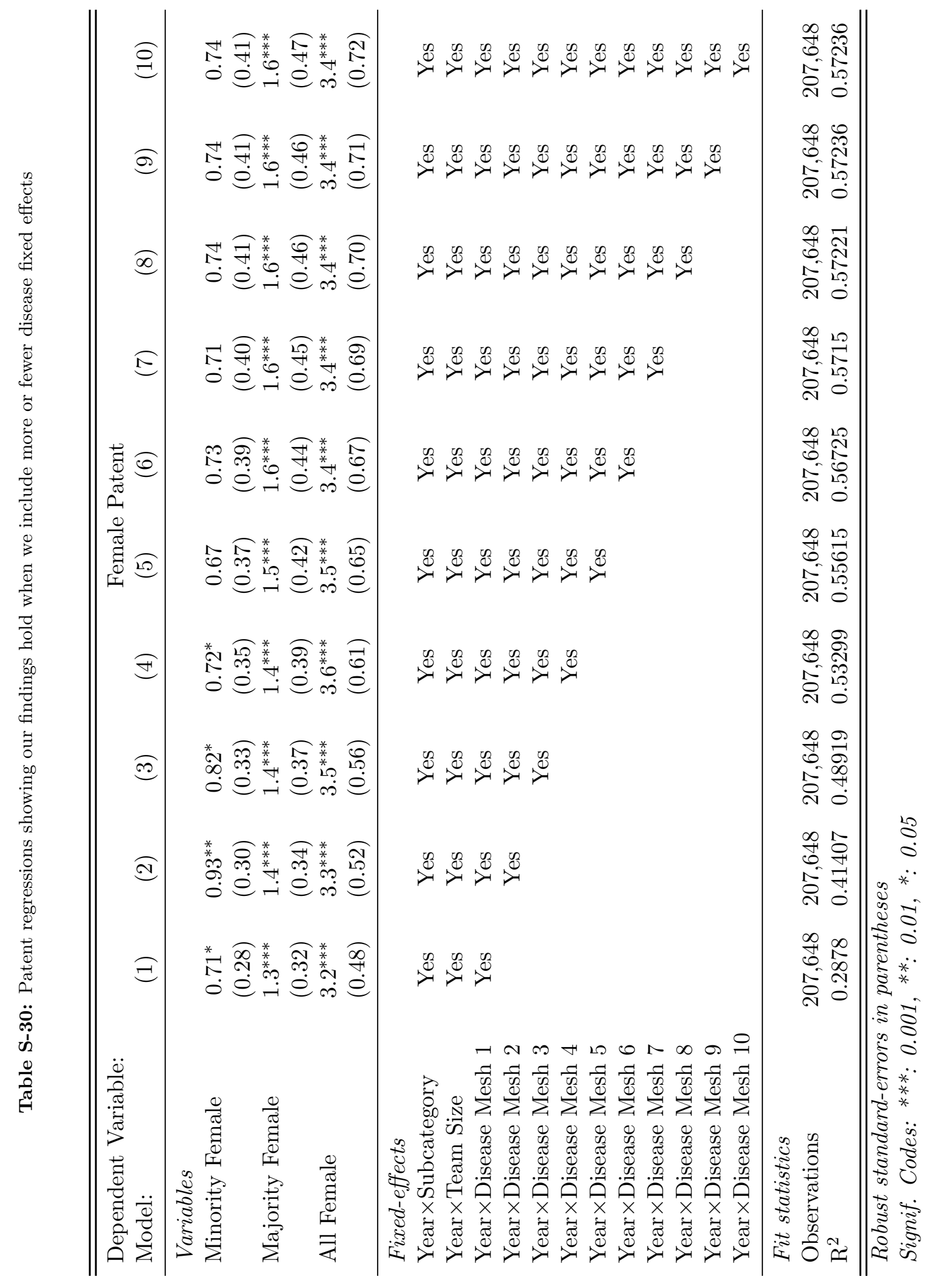


Table S-31: Patent regressions with double selection lasso show similar results

\begin{tabular}{|c|c|c|c|}
\hline $\begin{array}{l}\text { Dependent Variable: } \\
\text { Model: }\end{array}$ & \multicolumn{3}{|c|}{ Female Patent } \\
\hline \multicolumn{4}{|l|}{ Variables } \\
\hline Minority Female & $\begin{array}{l}1.9^{* * *} \\
(0.35)\end{array}$ & $\begin{array}{c}0.97^{* * *} \\
(0.21)\end{array}$ & $\begin{array}{c}0.65^{* * *} \\
(0.17)\end{array}$ \\
\hline Majority Female & $2.3^{* * *}$ & $1.1^{* * * *}$ & $1.7^{* * * *}$ \\
\hline All Female & $\begin{array}{l}5.5^{* * *} \\
(0.80)\end{array}$ & $\begin{array}{l}2.5^{* * *} \\
(0.46)\end{array}$ & $\begin{array}{l}3.5^{* * *} \\
(0.37)\end{array}$ \\
\hline \multicolumn{4}{|l|}{ Fixed Effects } \\
\hline Year×Team Size & Yes & Yes & Yes \\
\hline Year $\times$ Subcategory & Yes & Yes & Yes \\
\hline \multicolumn{4}{|l|}{ Sample Restrictions } \\
\hline Restriction & $1976-1989$ & 1990-1999 & $2000-2010$ \\
\hline Observations & 70,395 & 145,704 & 213,961 \\
\hline
\end{tabular}

Table S-32: Publication regressions corresponding to Figure 4B

\begin{tabular}{|c|c|c|c|c|}
\hline \multirow{2}{*}{$\begin{array}{l}\text { Dependent Variable: } \\
\text { Model: }\end{array}$} & \multicolumn{4}{|c|}{ Female Research } \\
\hline & (1) & $(2)$ & $(3)$ & $(4)$ \\
\hline \multicolumn{5}{|l|}{ Variables } \\
\hline Minority Female & $\begin{array}{c}0.84^{* * *} \\
(0.08)\end{array}$ & $\begin{array}{c}0.74^{* * *} \\
(0.17)\end{array}$ & & \\
\hline \multirow[t]{2}{*}{ Majority Female } & $3^{* * *}$ & & $2.4^{* * *}$ & \\
\hline & $(0.08)$ & & $(0.19)$ & \\
\hline \multirow[t]{2}{*}{ All Female } & $4.5^{* * *}$ & & & $4.1^{* * *}$ \\
\hline & $(0.12)$ & & & $(0.32)$ \\
\hline \multicolumn{5}{|l|}{ Fixed-effects } \\
\hline Year×Team Size & Yes & Yes & Yes & Yes \\
\hline Year $\times$ Journal & Yes & Yes & Yes & Yes \\
\hline Year $\times$ Team Size $\times$ Disease & & Yes & Yes & Yes \\
\hline \multicolumn{5}{|l|}{ Exact Matching } \\
\hline Year $\times$ Team Size $\times$ Disease & & Yes & Yes & Yes \\
\hline \multicolumn{5}{|l|}{ Fit statistics } \\
\hline Observations & $2,062,695$ & 372,222 & 291,986 & 119,650 \\
\hline $\mathrm{R}^{2}$ & 0.3632 & 0.39536 & 0.42926 & 0.48682 \\
\hline
\end{tabular}


Table S-33: The 100 words most associated with publications tagged with the "Female" check tag

\begin{tabular}{|c|c|c|c|c|c|}
\hline $\begin{array}{l}\text { Z-Score } \\
\text { Rank }\end{array}$ & Word & $\begin{array}{l}\text { Raw Odds-Ratio } \\
\text { (Weighted Z-Score) }\end{array}$ & $\begin{array}{c}\text { Z-Score } \\
\text { Rank }\end{array}$ & Word & $\begin{array}{l}\text { Raw Odds-Ratio } \\
\text { (Weighted Z-Score) }\end{array}$ \\
\hline 1 & $\operatorname{cin} 3$ & $1283.4(23)$ & 51 & postmenopausal & $49.1(41)$ \\
\hline 2 & preantral & $437.6(13)$ & 52 & placentas & $48.1(14)$ \\
\hline 3 & parametrial & $381.5(12)$ & 53 & cesarean & $47.9(16)$ \\
\hline 4 & colposcopy & $362.3(11)$ & 54 & granulosa & $46(21)$ \\
\hline 5 & rrso & $339.4(12)$ & 55 & mastectomy & $45.7(26)$ \\
\hline 6 & conization & $329.6(12)$ & 56 & ovariectomized & $45.5(22)$ \\
\hline 7 & anovulation & $328.2(11)$ & 57 & females & $45.4(69)$ \\
\hline 8 & pprom & $324(11)$ & 58 & trimester & $44.6(26)$ \\
\hline 9 & hysteroscopy & $288.9(11)$ & 59 & alnd & $43.8(13)$ \\
\hline 10 & preeclamptic & $270.7(11)$ & 60 & estrus & $43.7(13)$ \\
\hline 11 & z0011 & $259.5(10)$ & 61 & postpartum & $40.6(25)$ \\
\hline 12 & leep & $251.1(10)$ & 62 & eoc & $39.9(20)$ \\
\hline 13 & $\operatorname{cin} 2$ & $239.8(13)$ & 63 & dcis & $39.7(24)$ \\
\hline 14 & myometrial & $234.1(14)$ & 64 & $\mathrm{gdm}$ & $38.3(19)$ \\
\hline 15 & nulliparous & $216.8(11)$ & 65 & ovariectomy & $38.3(15)$ \\
\hline 16 & figo & $215.2(15)$ & 66 & genders & $38.2(15)$ \\
\hline 17 & $\mathrm{pcos}$ & $192.9(29)$ & 67 & catastrophizing & $38(10)$ \\
\hline 18 & endometrioid & $171.2(16)$ & 68 & menopausal & $37.5(24)$ \\
\hline 19 & endometriosis & $136.7(24)$ & 69 & women's & $37.4(20)$ \\
\hline 20 & woman & $132.1(43)$ & 70 & menopause & $35.1(22)$ \\
\hline 21 & hysterectomy & $128(21)$ & 71 & tubal & 34.1 (11) \\
\hline 22 & menarche & $120.1(13)$ & 72 & vaginal & $33.6(35)$ \\
\hline 23 & preeclampsia & $117.4(24)$ & 73 & obstetric & 32.7 (13) \\
\hline 24 & decidual & $106.8(12)$ & 74 & female & $31.6(97)$ \\
\hline 25 & myometrium & $100.8(12)$ & 75 & lumpectomy & $30.6(10)$ \\
\hline 26 & pregnancies & $99.9(31)$ & 76 & ovarian & $28.1(71)$ \\
\hline 27 & girl & $98.4(18)$ & 77 & gender & $27.6(65)$ \\
\hline 28 & fibroids & $96.3(10)$ & 78 & ovulation & $27.3(18)$ \\
\hline 29 & vulvar & 90.7 (13) & 79 & nstemi & $25.1(13)$ \\
\hline 30 & women & $88.2(160)$ & 80 & sexes & $23.3(24)$ \\
\hline 31 & luteal & $87.9(18)$ & 81 & uterus & $23.1(22)$ \\
\hline 32 & transvaginal & 87.9 (13) & 82 & ovaries & $22.9(20)$ \\
\hline 33 & premenopausal & $87.8(24)$ & 83 & cytoreduction & $22.7(13)$ \\
\hline 34 & ovx & $83.1(27)$ & 84 & ewes & $22.3(13)$ \\
\hline 35 & eclampsia & $80.6(12)$ & 85 & lvmi & $22.1(10)$ \\
\hline 36 & endometrial & 77.9 (44) & 86 & antenatal & $22.1(12)$ \\
\hline 37 & girls & $76.9(32)$ & 87 & ccrt & $21.6(10)$ \\
\hline 38 & oophorectomy & $69.7(12)$ & 88 & euroscore & $21.5(14)$ \\
\hline 39 & ovulatory & $66.2(11)$ & 89 & vagina & $21.2(12)$ \\
\hline 40 & miscarriage & $61.9(12)$ & 90 & $\operatorname{slnb}$ & $21.1(15)$ \\
\hline 41 & menstrual & $61.2(24)$ & 91 & mace & $20.8(24)$ \\
\hline 42 & endometrium & $60.8(21)$ & 92 & lactation & $20.3(19)$ \\
\hline 43 & estrous & $58.3(16)$ & 93 & savr & $20.1(10)$ \\
\hline 44 & uterine & $56.6(41)$ & 94 & nipple & $19.7(16)$ \\
\hline 45 & gynecologic & 55.4 (19) & 95 & mothers & $19.5(31)$ \\
\hline 46 & pregnant & $55(47)$ & 96 & mammographic & $19.2(18)$ \\
\hline 47 & pregnancy & $54.6(70)$ & 97 & gynecological & $19.1(11)$ \\
\hline 48 & nonpregnant & $53.5(15)$ & 98 & hrql & $18.9(12)$ \\
\hline 49 & fallopian & $50.2(12)$ & 99 & cervix & $18.8(17)$ \\
\hline 50 & amci & $49.4(11)$ & 100 & breasts & $18.6(16)$ \\
\hline
\end{tabular}


Table S-34: The 100 words most associated with publications tagged with the "Male" check tag

\begin{tabular}{|c|c|c|c|c|c|}
\hline $\begin{array}{l}\text { Z-Score } \\
\text { Rank }\end{array}$ & Word & $\begin{array}{l}\text { Raw Odds-Ratio } \\
\text { (Weighted Z-Score) }\end{array}$ & $\begin{array}{c}\text { Z-Score } \\
\text { Rank }\end{array}$ & Word & $\begin{array}{l}\text { Raw Odds-Ratio } \\
\text { (Weighted Z-Score) }\end{array}$ \\
\hline 1 & iief & $1029.2(21)$ & 51 & epididymis & $22.3(12)$ \\
\hline 2 & gleason & $979.2(30)$ & 52 & sexes & $21.4(23)$ \\
\hline 3 & hgpin & $665.8(17)$ & 53 & vasc & $21.3(11)$ \\
\hline 4 & psadt & $503.8(15)$ & 54 & hfref & $20.2(11)$ \\
\hline 5 & extraprostatic & $500(15)$ & 55 & ipss & $19.7(14)$ \\
\hline 6 & intraprostatic & 457.9 (14) & 56 & euroscore & $19.4(13)$ \\
\hline 7 & sextant & $423.4(13)$ & 57 & mace & $19.3(23)$ \\
\hline 8 & peyronie's & $292.1(11)$ & 58 & lncap & $19.1(23)$ \\
\hline 9 & intratesticular & $276.8(11)$ & 59 & rankin & $19(17)$ \\
\hline 10 & chordee & $251.3(10)$ & 60 & cto & $18.4(16)$ \\
\hline 11 & erspc & $250(10)$ & 61 & ahi & $18.3(19)$ \\
\hline 12 & gonocytes & $239.8(10)$ & 62 & abiraterone & $17.6(10)$ \\
\hline 13 & mcrpc & $202(10)$ & 63 & leydig & $17.3(14)$ \\
\hline 14 & varicocele & $192(12)$ & 64 & mmse & $17.2(15)$ \\
\hline 15 & seminiferous & $121.5(13)$ & 65 & sperm & $17.2(45)$ \\
\hline 16 & prostates & $120.5(10)$ & 66 & octogenarians & $16.6(10)$ \\
\hline 17 & turp & $118.7(11)$ & 67 & tavi & $16.5(20)$ \\
\hline 18 & scrotal & $116.1(12)$ & 68 & stemi & $15.8(27)$ \\
\hline 19 & prostatectomy & $102.6(33)$ & 69 & postexercise & $15.6(11)$ \\
\hline 20 & spermatogonia & $98.9(13)$ & 70 & tevar & $15.4(14)$ \\
\hline 21 & prostatitis & $97.7(13)$ & 71 & adt & $15.3(16)$ \\
\hline 22 & $\mathrm{crpc}$ & $83.3(16)$ & 72 & pugh & $15.2(14)$ \\
\hline 23 & penile & 80.9 (23) & 73 & hypopnea & $15.1(14)$ \\
\hline 24 & hypospadias & $77.6(13)$ & 74 & ohca & $15(15)$ \\
\hline 25 & spermatozoa & $73.5(22)$ & 75 & esophagectomy & $14.6(18)$ \\
\hline 26 & acrosome & $69(12)$ & 76 & $\mathrm{fvc}$ & $14.4(16)$ \\
\hline 27 & boy & 67.7 (19) & 77 & rehospitalization & $14.2(11)$ \\
\hline 28 & spermatids & $65.7(12)$ & 78 & beva & $14.2(23)$ \\
\hline 29 & prostatic & $64.3(31)$ & 79 & oswestry & $14.2(13)$ \\
\hline 30 & spermatogonial & $61.8(10)$ & 80 & nyha & $14(18)$ \\
\hline 31 & ejaculation & $60.9(11)$ & 81 & periprocedural & $13.8(14)$ \\
\hline 32 & boys & $57.5(28)$ & 82 & updrs & $13.7(12)$ \\
\hline 33 & castration & 48.4 (19) & 83 & clavien & $13.6(11)$ \\
\hline 34 & spermatocytes & $45.5(12)$ & 84 & midterm & $13.6(15)$ \\
\hline 35 & penis & $45(12)$ & 85 & ppci & $13.5(11)$ \\
\hline 36 & testicular & $44(32)$ & 86 & charlson & $13.3(14)$ \\
\hline 37 & sertoli & $43.1(18)$ & 87 & womac & $13.2(11)$ \\
\hline 38 & amci & $43.1(10)$ & 88 & cabg & $13(34)$ \\
\hline 39 & erectile & $40.2(23)$ & 89 & fev1 & $13(19)$ \\
\hline 40 & males & $37.7(66)$ & 90 & handgrip & $12.9(10)$ \\
\hline 41 & genders & $32.6(15)$ & 91 & wmh & $12.8(11)$ \\
\hline 42 & castrated & $32(11)$ & 92 & psma & $12.4(17)$ \\
\hline 43 & lvmi & $31.1(10)$ & 93 & cimt & $12.2(14)$ \\
\hline 44 & prostate & $29.4(101)$ & 94 & subfoveal & $12(12)$ \\
\hline 45 & male & $26.2(105)$ & 95 & holter & $12(13)$ \\
\hline 46 & spermatogenesis & $25.1(19)$ & 96 & ewl & $11.8(11)$ \\
\hline 47 & epididymal & $24.5(15)$ & 97 & das 28 & $11.8(13)$ \\
\hline 48 & nstemi & $23.2(12)$ & 98 & sua & $11.7(10)$ \\
\hline 49 & testes & $22.8(20)$ & 99 & tavr & $11.7(16)$ \\
\hline 50 & gender & $22.8(61)$ & 100 & nihss & $11.6(13)$ \\
\hline
\end{tabular}


Table S-35: Papers tagged as female address diseases with higher female incidence counts; male tagged papers address diseases with higher male incidence

\begin{tabular}{|c|c|c|c|c|}
\hline \multirow{2}{*}{$\begin{array}{l}\text { Dependent Variables: } \\
\text { Model: }\end{array}$} & \multicolumn{2}{|c|}{ Log Female Incidence } & \multicolumn{2}{|c|}{ Log Male Incidence } \\
\hline & (1) & $(2)$ & (3) & $(4)$ \\
\hline \multicolumn{5}{|l|}{ Variables } \\
\hline Female MeSH & $\begin{array}{c}2^{* * *} \\
(0.009)\end{array}$ & $\begin{array}{l}3.4^{* * *} \\
(0.01)\end{array}$ & & $\begin{array}{c}-3.5^{* * *} \\
(0.01)\end{array}$ \\
\hline Male MeSH & & $\begin{array}{c}-3.5^{* * *} \\
(0.01)\end{array}$ & $\begin{array}{c}2.1^{* * *} \\
(0.008)\end{array}$ & $\begin{array}{l}3.5^{* * *} \\
(0.01)\end{array}$ \\
\hline Log Male Incidence & $\begin{array}{l}0.72^{* * *} \\
(0.001)\end{array}$ & $\begin{array}{c}0.79^{* * *} \\
(0.0010)\end{array}$ & & \\
\hline Log Female Incidence & & & $\begin{array}{l}0.72^{* * *} \\
(0.001)\end{array}$ & $\begin{array}{l}0.78^{* * *} \\
(0.001)\end{array}$ \\
\hline (Intercept) & $\begin{array}{l}1.9^{* * *} \\
(0.01)\end{array}$ & $\begin{array}{l}2.2^{* * *} \\
(0.01)\end{array}$ & $\begin{array}{l}1.7^{* * *} \\
(0.02)\end{array}$ & $\begin{array}{l}2.3^{* * *} \\
(0.01)\end{array}$ \\
\hline \multicolumn{5}{|l|}{ Fit statistics } \\
\hline Observations & 581,831 & 581,831 & 581,831 & 581,831 \\
\hline $\mathrm{R}^{2}$ & 0.54488 & 0.64336 & 0.54912 & 0.64785 \\
\hline
\end{tabular}


Table S-36: List of publications with our female-only-trial prediction measure

\begin{tabular}{|c|c|c|c|}
\hline P(Female Trial) & P(Male Trial) & Patent title & Female Focused? \\
\hline $100 \%$ & $0 \%$ & Multiparity and the risk of premenopausal breast cancer: different effects across ethnic groups in Singapore. & $\mathrm{Y}$ \\
\hline $100 \%$ & $0 \%$ & Screening mammography in older women: a review. & $\mathrm{Y}$ \\
\hline $100 \%$ & $0 \%$ & Vaginal mesh extrusion associated with use of Mentor transobturator sling. & $\mathrm{Y}$ \\
\hline $98 \%$ & $0 \%$ & Expression of Toll-like receptors 2, 4 and 6 in the equine chorioallantois. & \\
\hline $97 \%$ & $0 \%$ & Female adolescents' views on a youth-friendly clinic. & $\mathrm{Y}$ \\
\hline $90 \%$ & $0 \%$ & Use of Six Sigma Methodology to Reduce Appointment Lead-Time in Obstetrics Outpatient Department. & $\mathrm{Y}$ \\
\hline $89 \%$ & $0 \%$ & Denosumab and teriparatide transitions in postmenopausal osteoporosis (the DATA-Switch study): extension .... & $\mathrm{Y}$ \\
\hline $88 \%$ & $1 \%$ & MiR-22I-3p is down-regulated in preeclampsia and affects trophoblast growth, invasion and ... & $\mathrm{Y}$ \\
\hline $87 \%$ & $0 \%$ & Docosahexaenoic and arachidonic acid concentrations in human breast milk worldwide. & $\mathrm{Y}$ \\
\hline $81 \%$ & $2 \%$ & Neuronal microtubule-associated protein $2 D$ is a dual a-kinase anchoring protein expressed in rat ovarian ... & \\
\hline $77 \%$ & $1 \%$ & Neonatal vaginal irritation results in long-term visceral and somatic hypersensitivity ... & $\mathrm{Y}$ \\
\hline $76 \%$ & $3 \%$ & Alkaloids and flavonoid glycosides from the aerial parts of Leonurus japonicus and their opposite effects on uterin & $\mathrm{Y}$ \\
\hline $75 \%$ & $2 \%$ & Fas-mediated pathway and apoptosis in normal, hyperplastic, and neoplastic endometrium. & Y \\
\hline $71 \%$ & $1 \%$ & Effect of branched-chain fatty acids on fatty acid biosynthesis of human breast cancer cells. & $\mathrm{Y}$ \\
\hline $71 \%$ & $9 \%$ & Posttranscriptional control of the competence factor betaFTZ-FI by juvenile hormone in the mosquito Aedes aegypti. & \\
\hline $67 \%$ & $5 \%$ & Effects of ketamine on the fetal transcriptomic response to umbilical cord occlusion: comparison with ... & \\
\hline $67 \%$ & $39 \%$ & The effect of individual and mixtures of mycotoxins and persistent organochloride pesticides on oestrogen ... & \\
\hline $66 \%$ & $0 \%$ & Complications after autologous fat injection to the breast. & $\mathrm{Y}$ \\
\hline $65 \%$ & $1 \%$ & Evaluation of loading parameters for murine axial tibial loading: Stimulating cortical bone formation while ... & \\
\hline $63 \%$ & $0 \%$ & Pregnancy outcomes after maternal exposure to rituximab. & $\mathrm{Y}$ \\
\hline $59 \%$ & $3 \%$ & Angiopoietin-1 and vascular endothelial growth factor regulation of leukocyte adhesion to ... & \\
\hline $57 \%$ & $0 \%$ & Frontal fibrosing alopecia in postmenopausal women. & Y \\
\hline $55 \%$ & $0 \%$ & Predicting non-home discharge in epithelial ovarian cancer patients: External validation of a predictive model. & $\mathrm{Y}$ \\
\hline $53 \%$ & $0 \%$ & The association between smoking and breast cancer characteristics and outcome. & $\mathrm{Y}$ \\
\hline $52 \%$ & $2 \%$ & Combined trastuzumab and paclitaxel treatment better inhibits ErbB-2-mediated angiogenesis in breast $c \ldots$ & $\mathrm{Y}$ \\
\hline $48 \%$ & $11 \%$ & Uptake of dietary retinoids at the maternal-fetal barrier: in vivo evidence for the role of lipoprotein lipase and ... & $\mathrm{Y}$ \\
\hline $47 \%$ & $0 \%$ & Peri-operative allogeneic blood transfusion is associated with poor overall survival in advanced ... & \\
\hline $46 \%$ & $0 \%$ & $c k 2$-dependent phosphorylation of progesterone receptors $(P R)$ on Ser81 regulates $P R-B$ isoform-specific target ... & \\
\hline $44 \%$ & $0 \%$ & Adaptive neuro-fuzzy inference systems for automatic detection of breast cancer. & $\mathrm{Y}$ \\
\hline $43 \%$ & $16 \%$ & Selective cytotoxicity of a Vietnamese traditional formula, Nam Dia long, against $M C F-7$ cells by synergistic effects. & \\
\hline $39 \%$ & $93 \%$ & An efficient synthesis of 5alpha-androst-I-ene-3,17-dione. & \\
\hline $38 \%$ & $1 \%$ & Protein phosphatase 3 differentially modulates vascular endothelial growth factor- and fibroblast growth factor... & \\
\hline $38 \%$ & $1 \%$ & Prospective evaluation of the Hybrid Capture 2 and AMPLICOR human papillomavirus (HPV) tests for detection of $13 \ldots$ & \\
\hline $32 \%$ & $0 \%$ & ADA3 regulates normal and tumor mammary epithelial cell proliferation through c-MYC. & Y \\
\hline $31 \%$ & $0 \%$ & Heterogeneous expression of GAGE, NY-ESO-1, MAGE-A and SSX proteins in esophageal cancer: ... & \\
\hline $28 \%$ & $30 \%$ & Steroid receptor expression in the fish inner ear varies with sex, social status, and reproductive state. & \\
\hline $28 \%$ & $1 \%$ & Fragmentation and development of preimplantation porcine embryos derived by parthenogenetic activation and ... & \\
\hline $28 \%$ & $0 \%$ & Renal angiomyolipomas from patients with sporadic lymphangiomyomatosis contain both neoplastic ... & \\
\hline $24 \%$ & $0 \%$ & Severe aortic insufficiency after transapical aortic valve implantation. & \\
\hline $23 \%$ & $4 \%$ & Transcriptome profiling of genes involving in carotenoid biosynthesis and accumulation between leaf $a \ldots$ & \\
\hline $17 \%$ & $1 \%$ & mPGES1-Dependent Prostaglandin E2 (PGE2) Controls Antigen-Specific Th17 and Thl Responses by... & \\
\hline $15 \%$ & $43 \%$ & Biosynthesis of the beta-amino acid moiety of the enediyne antitumor antibiotic C-1027 featuring beta-amino ... & \\
\hline $14 \%$ & $0 \%$ & High commitment of embryonic keratinocytes to terminal differentiation through a Notchl-caspase $3 \ldots$ & \\
\hline $12 \%$ & $19 \%$ & Stellate ganglion block ameliorates vascular calcification by inhibiting endoplasmic reticulum stress. & \\
\hline $12 \%$ & $19 \%$ & Progress Toward a Semi-Synthetic Organism with an Unrestricted Expanded Genetic Alphabet. & \\
\hline $4 \%$ & $20 \%$ & Halimium halimifolium: From the Chemical and Functional Characterization to a Nutraceutical Ingredient Design. & \\
\hline $4 \%$ & $6 \%$ & Discovery of new [1,4]dioxino[2,3-f]quinazoline-based inhibitors of EGFR including the T790M/L858R mutant. & \\
\hline $3 \%$ & $5 \%$ & Low prevalence of antiretroviral resistance among HIV type 1-positive prisoners in the Southeast United States. & \\
\hline $2 \%$ & $10 \%$ & $R T-P C R$ permits simultaneous genotyping of thiopurine S-methyltransferase allelic variants by multiple... & \\
\hline $1 \%$ & $1 \%$ & Prolonged cyclosporine administration after antithymocyte globulin delays but does not prevent relapse in... & \\
\hline
\end{tabular}


Table S-37: List of publications with our male-only-trial prediction measure

\begin{tabular}{|c|c|c|c|}
\hline $\mathbf{P}($ Female Trial $)$ & P(Male Trial) & Patent title & Male Focused? \\
\hline $0 \%$ & $100 \%$ & Augmentation of $T$ cell levels and responses induced by androgen deprivation. & $\mathrm{Y}$ \\
\hline $0 \%$ & $98 \%$ & Estrogen blunts neuroendocrine and metabolic responses to hypoglycemia. & \\
\hline $0 \%$ & $97 \%$ & How sensitive is a prostate-specific antigen measurement? How sensitive does it need to $b$ & Y \\
\hline $0 \%$ & $97 \%$ & Insulin-like growth factor 1, chromogranin A and prostate specific antigen serum levels. & $\mathrm{Y}$ \\
\hline $0 \%$ & $96 \%$ & Emergence and persistence of CXCR4-tropic HIV-1 in a population of men from... & $\mathrm{Y}$ \\
\hline $0 \%$ & $90 \%$ & No Association between the Mitochondrial Genome and Prostate Cancer Risk: ... & $\mathrm{Y}$ \\
\hline $6 \%$ & $89 \%$ & Magnetic resonance imaging is a complementary method to stereological measurement o. & Y \\
\hline $1 \%$ & $85 \%$ & The effects in mice of combined treatments to $X$-rays and antineoplastic drugs in the Comet & ssay. \\
\hline $0 \%$ & $84 \%$ & Prostate cancer-specific monoclonal antibody $5 D 4$ significantly enhances the cytotoxicit & $\mathrm{Y}$ \\
\hline $0 \%$ & $84 \%$ & PEDF protects cardiomyocytes by promoting $F U N D C 1$, Äëmediated mitophagy via PEDH & $\mathrm{Y}$ \\
\hline $0 \%$ & $80 \%$ & Pharmacokinetics, disposition, and metabolism of bicifadine in humans. & \\
\hline $8 \%$ & $75 \%$ & The respiratory chain of the thermophilic archaeon Sulfolobus metallicus: studies on the... & \\
\hline $0 \%$ & $75 \%$ & Association of genetic variations of selenoprotein genes, plasma selenium levels, and pro & Y \\
\hline $4 \%$ & $73 \%$ & Absence of Bcl-2 expression favors response to the short-term administration of diethylsti & Y \\
\hline $4 \%$ & $73 \%$ & Carbenoid reactions of 2-halomethyl-4,6-dimethyl-s-triazines. & \\
\hline $0 \%$ & $67 \%$ & Brefeldin A inhibits cholesterol efflux without affecting the rate of cellular uptake and ... & \\
\hline $8 \%$ & $64 \%$ & Mitochondrial oxidant stress triggers cell death in simulated ischemia-reperfusion. & \\
\hline $4 \%$ & $62 \%$ & Cleavage and synthesis function of high and low redox potential laccases towards 4-morph & inoaniline ... \\
\hline $0 \%$ & $62 \%$ & The Ceratopteris (fern) developing motile gamete walls contain diverse $\neg$ polysaccharides, $b$ & t not pectin. \\
\hline $1 \%$ & $62 \%$ & Identification and expression of mangrove rivulus (Kryptolebias marmoratus) histone deace & lase... \\
\hline $3 \%$ & $59 \%$ & Efficacy of sildenafil as adjuvant therapy... inhibitor in alleviating premature ejaculation & $\mathrm{Y}$ \\
\hline $2 \%$ & $55 \%$ & Characterization of the mechanism and magnitude of cytoglobin-mediated nitrite reduction & \\
\hline $3 \%$ & $54 \%$ & Estimation of the hydrophobicity of 2,4-diphenyl-1,3-oxazoline analogs and QSAR analysi & $f f . .$. \\
\hline $6 \%$ & $53 \%$ & COSMO-RS for the prediction of the retention behavior in micellar liquid chromatography & Ised ... \\
\hline $6 \%$ & $52 \%$ & Western Diet Triggers NLRP3-Dependent Innate Immune Reprogramming. & \\
\hline $1 \%$ & $48 \%$ & Duration of action of a broad range of selective Ef-opioid receptor antagonists is positivel] & \\
\hline $6 \%$ & $47 \%$ & Continuous 24-h nicotinic acid infusion in rats causes FFA rebound and insulin resistance & \\
\hline $7 \%$ & $47 \%$ & Flavin-containing enzymes as a source of reactive oxygen species in HEMA-induced apopte & \\
\hline $7 \%$ & $43 \%$ & A highly specific and ultrasensitive probe for the imaging of inflammation-induced endoger & us ... \\
\hline $2 \%$ & $42 \%$ & Shear stress-induced mitochondrial biogenesis decreases the release of microparticles from & dothelial cells. \\
\hline $3 \%$ & $39 \%$ & Aorta and liver changes in rats fed cholesterol-containing and raw vegetable-supplemented & liets:... \\
\hline $0 \%$ & $35 \%$ & Tumor length in prostate cancer. & Y \\
\hline $13 \%$ & $33 \%$ & Structure-activity relationships of peptides incorporating a bioactive reverse-turn heterocyc & \\
\hline $3 \%$ & $33 \%$ & Correlation of $p Q C T$ bone strength index with mechanical testing in distraction osteogenesi & \\
\hline $1 \%$ & $31 \%$ & Essential role of the cAMP-cAMP response-element binding protein pathway in opiate-indu & ed ... \\
\hline $9 \%$ & $28 \%$ & Synthesis and biological evaluation of 7-O-modified oroxylin A derivatives. & \\
\hline $0 \%$ & $28 \%$ & Game of 'Somes: Protein Destruction for Mycobacterium tuberculosis Pathogenesis. & \\
\hline $5 \%$ & $27 \%$ & Structures of Leishmania major pteridine reductase complexes reveal the active site features & \\
\hline $1 \%$ & $23 \%$ & GTP binding and hydrolysis kinetics of human septin 2. & \\
\hline $9 \%$ & $22 \%$ & Association of DRD2, 5-HTTLPR, and 5-HTTVNTR Gene Polymorphisms With Posttraumat & Stress. \\
\hline $2 \%$ & $19 \%$ & Near-native structure refinement using in vacuo energy minimization. & \\
\hline $12 \%$ & $16 \%$ & Proteome profile of the developing maize (Zea mays L.) rachis. & \\
\hline $4 \%$ & $15 \%$ & GUCY2C Signaling Opposes the Acute Radiation-Induced GI Syndrome. & \\
\hline $4 \%$ & $13 \%$ & Piracetam, an AMPAkine drug, facilitates memory consolidation in the day-old chick. & \\
\hline $3 \%$ & $11 \%$ & Precision is essential for efficient catalysis in an evolved Kemp eliminase. & \\
\hline $0 \%$ & $8 \%$ & Structural investigation of the HIV-1 envelope glycoprotein gp 160 cleavage site. & \\
\hline $2 \%$ & $5 \%$ & Cystic fibrosis transmembrane conductance regulator: a molecular model defines the... & \\
\hline $1 \%$ & $3 \%$ & Scintigraphic detection of pulmonary aspergillosis in rabbits with a radiolabeled leukotrien & $b 4 \ldots$ \\
\hline $0 \%$ & $1 \%$ & An Exploratory Study of Dapagliflozin for the Attenuation of Albuminuria in Patients with $\mathrm{I}$ & eart ... \\
\hline $0 \%$ & $0 \%$ & Aeromonas species: an opportunistic enteropathogen in patients with inflammatory bowel d & eases?.. \\
\hline
\end{tabular}




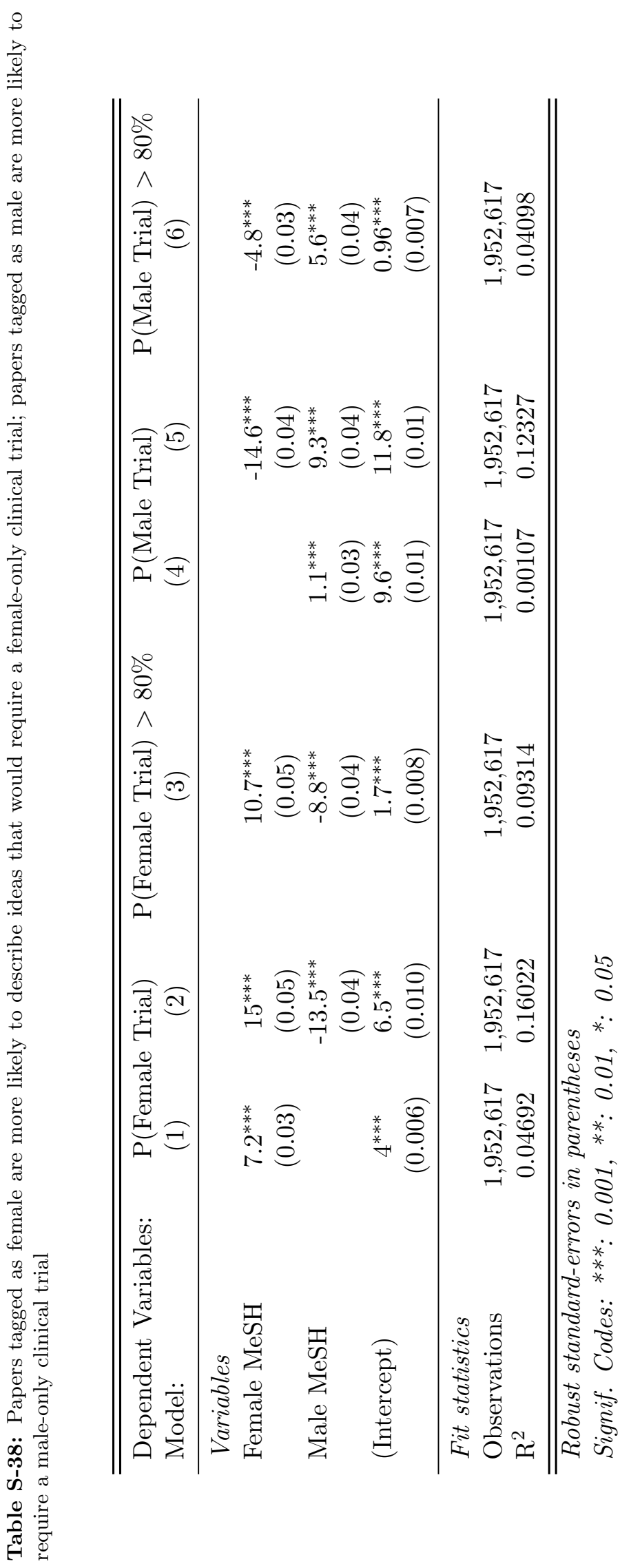


Table S-39: Publications by women are not more likely to focus on the medical needs of men

\begin{tabular}{|c|c|c|c|c|}
\hline $\begin{array}{l}\text { Dependent Variable: } \\
\text { Model: }\end{array}$ & (1) & $\begin{array}{l}\text { Male 1 } \\
(2)\end{array}$ & $\begin{array}{l}\text { Research } \\
\qquad(3)\end{array}$ & $(4)$ \\
\hline \multicolumn{5}{|l|}{ Variables } \\
\hline Minority Female & $\begin{array}{c}-0.28^{* * *} \\
(0.08)\end{array}$ & $\begin{array}{c}-0.90^{* * *} \\
(0.16)\end{array}$ & & \\
\hline Majority Female & $\begin{array}{c}-0.94^{* * *} \\
(0.08)\end{array}$ & & $\begin{array}{c}-1.3^{* * *} \\
(0.18)\end{array}$ & \\
\hline All Female & $\begin{array}{c}0.24 \\
(0.13)\end{array}$ & & & $\begin{array}{l}0.80^{*} \\
(0.32)\end{array}$ \\
\hline \multicolumn{5}{|l|}{ Fixed-effects } \\
\hline Year×Team Size & Yes & Yes & Yes & Yes \\
\hline Year $\times$ Disease & & Yes & Yes & Yes \\
\hline Year $\times$ Journal & Yes & Yes & Yes & Yes \\
\hline Year $\times$ Team Size $\times$ Disease & & Yes & Yes & Yes \\
\hline \multicolumn{5}{|l|}{ Exact Matching } \\
\hline Year $\times$ Team Size $\times$ Disease & & Yes & Yes & Yes \\
\hline \multicolumn{5}{|l|}{ Fit statistics } \\
\hline Observations & $2,062,695$ & 372,222 & 291,986 & 119,650 \\
\hline $\mathrm{R}^{2}$ & 0.36702 & 0.40399 & 0.43772 & 0.50051 \\
\hline
\end{tabular}


Table S-40: Women are more likely to discover for women regardless of the Journal Commercialization Impact Factor (JCIF) of the journal where the article is published

\begin{tabular}{|c|c|c|c|c|c|c|c|c|}
\hline \multirow{2}{*}{$\begin{array}{l}\text { Dependent Variable: } \\
\text { Model: }\end{array}$} & \multicolumn{8}{|c|}{ Female Research } \\
\hline & (1) & $(2)$ & (3) & (4) & (5) & (6) & $(7)$ & (8) \\
\hline \multicolumn{9}{|l|}{ Variables } \\
\hline \multirow[t]{2}{*}{ Minority Female } & $1.1^{* * *}$ & $0.89^{* * *}$ & $0.84^{* * *}$ & $0.94^{* * *}$ & $0.52^{* * *}$ & $0.80^{* * *}$ & $0.73^{* * *}$ & 0.53 \\
\hline & $(0.05)$ & $(0.08)$ & $(0.08)$ & $(0.13)$ & $(0.10)$ & $(0.20)$ & $(0.19)$ & $(0.57)$ \\
\hline \multirow[t]{2}{*}{ Majority Female } & $3.5^{* * *}$ & $2.4^{* * *}$ & $3^{* * *}$ & $2.2^{* * *}$ & $2.7^{* * *}$ & $2^{* * *}$ & $3.9^{* * *}$ & $2.9^{* * *}$ \\
\hline & $(0.05)$ & $(0.08)$ & $(0.08)$ & $(0.14)$ & $(0.10)$ & $(0.21)$ & $(0.20)$ & $(0.59)$ \\
\hline \multirow[t]{2}{*}{ All Female } & $5.2^{* * *}$ & $4.2^{* * *}$ & $4.5^{* * *}$ & $4.1^{* * *}$ & $3.8^{* * *}$ & $3.8^{* * *}$ & $4.2^{* * *}$ & $3.3^{* * *}$ \\
\hline & $(0.07)$ & $(0.11)$ & $(0.12)$ & $(0.22)$ & $(0.16)$ & $(0.35)$ & $(0.33)$ & $(0.96)$ \\
\hline \multicolumn{9}{|l|}{ Fixed-effects } \\
\hline Year $\times$ Team Size & Yes & Yes & Yes & Yes & Yes & Yes & Yes & Yes \\
\hline Year $\times$ Journal & Yes & Yes & Yes & Yes & Yes & Yes & Yes & Yes \\
\hline Year $\times$ Disease Area & & Yes & & Yes & & Yes & & Yes \\
\hline \multicolumn{9}{|l|}{ Sample Restrictions } \\
\hline JCIF Cut & All & All & 1,000 & 1,000 & 500 & 500 & 100 & 100 \\
\hline \multicolumn{9}{|l|}{ Fit statistics } \\
\hline Observations & $5,529,360$ & $2,727,870$ & $2,062,695$ & 975,653 & $1,121,807$ & 447,171 & 240,848 & 74,212 \\
\hline $\mathrm{R}^{2}$ & 0.36365 & 0.30799 & 0.3632 & 0.34524 & 0.32719 & 0.37344 & 0.22874 & 0.4465 \\
\hline
\end{tabular}


Table S-41: The findings in Figure 4B hold when match on journal instead of disease area

\begin{tabular}{|c|c|c|c|c|c|c|}
\hline \multirow{2}{*}{$\begin{array}{l}\text { Dependent Variable: } \\
\text { Model: }\end{array}$} & \multicolumn{6}{|c|}{ Female Research } \\
\hline & (1) & $(2)$ & (3) & $(4)$ & $(5)$ & (6) \\
\hline \multicolumn{7}{|l|}{ Variables } \\
\hline Minority Female & $\begin{array}{l}1.2^{* * *} \\
(0.10)\end{array}$ & & & $\begin{array}{c}0.77^{* * *} \\
(0.16)\end{array}$ & & \\
\hline Majority Female & & $\begin{array}{l}3.3^{* * *} \\
(0.10)\end{array}$ & & & $\begin{array}{l}2.5^{* * *} \\
(0.18)\end{array}$ & \\
\hline All Female & & & $\begin{array}{l}4.6^{* * *} \\
(0.14)\end{array}$ & & & $\begin{array}{l}3.8^{* * *} \\
(0.34)\end{array}$ \\
\hline \multicolumn{7}{|l|}{ Fixed-effects } \\
\hline Year $\times$ Team Size & Yes & Yes & Yes & Yes & Yes & Yes \\
\hline Year $\times$ Disease & & & & Yes & Yes & Yes \\
\hline Year $\times$ Journal & Yes & Yes & Yes & Yes & Yes & Yes \\
\hline Year $\times$ Team Size $\times$ Journal & Yes & Yes & Yes & Yes & Yes & Yes \\
\hline \multicolumn{7}{|l|}{ Exact Matching } \\
\hline Year $\times$ Team Size $\times$ Journal & Yes & Yes & Yes & Yes & Yes & Yes \\
\hline \multicolumn{7}{|l|}{ Fit statistics } \\
\hline Observations & 979,340 & 891,618 & 462,530 & 469,294 & 381,101 & 170,918 \\
\hline $\mathrm{R}^{2}$ & 0.40456 & 0.40502 & 0.4001 & 0.42092 & 0.45423 & 0.52368 \\
\hline
\end{tabular}

Robust standard-errors in parentheses. All columns are matching weighted.

Signif. Codes: ***: 0.001, **: 0.01, *: 0.05 
Table S-42: Publication regressions testing heterogeneity by time-period and authorship order

\begin{tabular}{|c|c|c|c|}
\hline \multirow{2}{*}{$\begin{array}{l}\text { Dependent Variable: } \\
\text { Model: }\end{array}$} & \multicolumn{3}{|c|}{ Female Research } \\
\hline & $(1)$ & $(2)$ & $(3)$ \\
\hline \multicolumn{4}{|l|}{ Variables } \\
\hline Minority Female & $\begin{array}{l}1.5^{* * *} \\
(0.17)\end{array}$ & $\begin{array}{l}1.4^{* * *} \\
(0.21)\end{array}$ & $\begin{array}{l}1.7^{* * *} \\
(0.29)\end{array}$ \\
\hline \multirow[t]{2}{*}{ Majority Female } & $2.4^{* * *}$ & $2.4^{* * *}$ & $2.3^{* * *}$ \\
\hline & $(0.26)$ & $(0.35)$ & $(0.42)$ \\
\hline \multirow[t]{2}{*}{ All Female } & $7.0^{* * *}$ & $6.9^{* * *}$ & $7.1^{* * *}$ \\
\hline & $(0.34)$ & $(0.44)$ & $(0.52)$ \\
\hline \multirow[t]{2}{*}{ Minority Female $\times$ Female First Author } & 0.23 & $0.49^{*}$ & -0.17 \\
\hline & $(0.17)$ & $(0.22)$ & $(0.26)$ \\
\hline \multirow[t]{2}{*}{ Minority Female $\times$ Female Last Author } & 0.24 & 0.16 & 0.35 \\
\hline & $(0.22)$ & $(0.29)$ & $(0.33)$ \\
\hline \multirow[t]{2}{*}{ Majority Female $\times$ Female First Author } & $0.63^{* *}$ & $0.77^{*}$ & 0.45 \\
\hline & $(0.23)$ & $(0.31)$ & $(0.35)$ \\
\hline \multirow[t]{2}{*}{ Majority Female×Female Last Author } & $1.1^{* * *}$ & $1.1^{* * *}$ & $1.2^{* * *}$ \\
\hline & $(0.22)$ & $(0.30)$ & $(0.32)$ \\
\hline Sample Restriction & & $2002-2012$ & $2013-2020$ \\
\hline \multicolumn{4}{|l|}{ Fixed-effects } \\
\hline Year×Journal & Yes & Yes & Yes \\
\hline Year×Team Size & Yes & Yes & Yes \\
\hline Year $\times$ Disease Area & Yes & Yes & Yes \\
\hline \multicolumn{4}{|l|}{ Sample Restrictions } \\
\hline Restriction & & $2002-2012$ & $2013-2020$ \\
\hline \multicolumn{4}{|l|}{ Fit statistics } \\
\hline Observations & 679,559 & 423,482 & 256,077 \\
\hline $\mathrm{R}^{2}$ & 0.35926 & 0.34039 & 0.3851 \\
\hline
\end{tabular}

Robust standard-errors in parentheses

Signif. Codes: ***: 0.001, **: 0.01, *: 0.05 


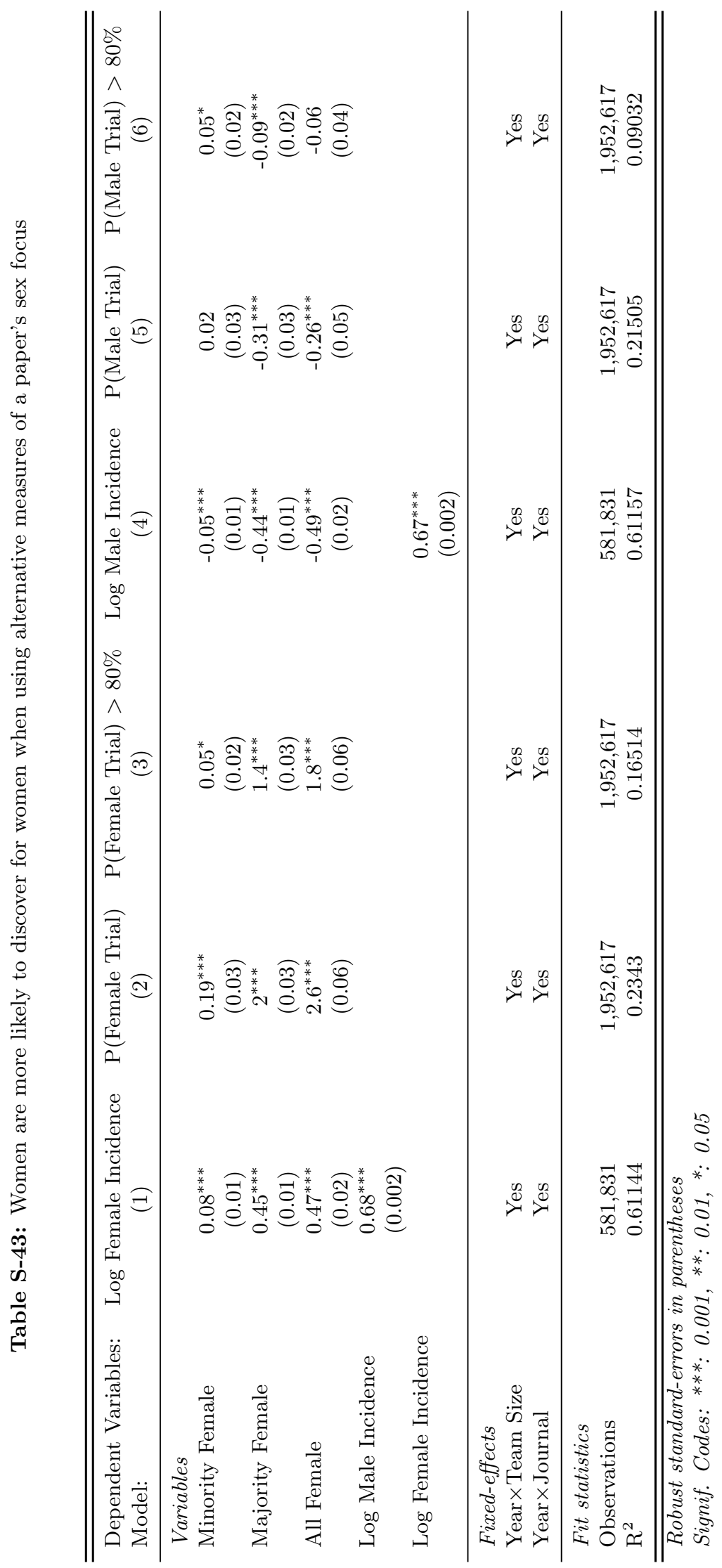


Table S-44: The findings in Figure 4B of paper hold when we instead use our clinical-trial measure of a paper's sex focus

\begin{tabular}{|c|c|c|c|c|}
\hline \multirow{2}{*}{$\begin{array}{l}\text { Dependent Variable: } \\
\text { Model: }\end{array}$} & \multicolumn{4}{|c|}{ Female Clinical Trial Research } \\
\hline & (1) & $(2)$ & (3) & $(4)$ \\
\hline \multicolumn{5}{|l|}{ Variables } \\
\hline Minority Female & $\begin{array}{c}0.19^{* * *} \\
(0.03)\end{array}$ & $\begin{array}{c}0.72^{* * *} \\
(0.05)\end{array}$ & & \\
\hline Majority Female & $\begin{array}{c}2^{* * *} \\
(0.03)\end{array}$ & & $\begin{array}{l}2.5^{* * *} \\
(0.08)\end{array}$ & \\
\hline All Female & $\begin{array}{l}2.6^{* * *} \\
(0.06)\end{array}$ & & & $\begin{array}{l}3.2^{* * *} \\
(0.15)\end{array}$ \\
\hline \multicolumn{5}{|l|}{ Fixed-effects } \\
\hline Year×Team Size & Yes & Yes & Yes & Yes \\
\hline Year $\times$ Disease & & Yes & Yes & Yes \\
\hline Year $\times$ Journal & Yes & Yes & Yes & Yes \\
\hline Year $\times$ Team Size $\times$ Disease & & Yes & Yes & Yes \\
\hline \multicolumn{5}{|l|}{ Exact Matching } \\
\hline Year $\times$ Team Size $\times$ Disease & & Yes & Yes & Yes \\
\hline \multicolumn{5}{|l|}{ Fit statistics } \\
\hline Observations & $1,952,617$ & 354,733 & 273,389 & 105,649 \\
\hline $\mathrm{R}^{2}$ & 0.2343 & 0.49746 & 0.52655 & 0.58685 \\
\hline
\end{tabular}


Table S-45: Patents by women are more likely to commercialize female focused papers

\begin{tabular}{|c|c|c|c|c|}
\hline \multirow{2}{*}{$\begin{array}{l}\text { Dependent Variables: } \\
\text { Family } \\
\text { Model: }\end{array}$} & \multicolumn{2}{|c|}{ Patent-Paper Pair } & \multicolumn{2}{|c|}{ NIH Grant } \\
\hline & $\begin{array}{c}\text { OLS } \\
(1)\end{array}$ & $\begin{array}{c}\text { Logit } \\
(2)\end{array}$ & $\begin{array}{c}\text { OLS } \\
(3)\end{array}$ & $\begin{array}{c}\text { Logit } \\
(4)\end{array}$ \\
\hline \multicolumn{5}{|l|}{ Variables } \\
\hline Minority Female & $\begin{array}{c}1.5^{*} \\
(0.76)\end{array}$ & $\begin{array}{c}0.10^{*} \\
(0.05)\end{array}$ & $\begin{array}{c}1.7 \\
(0.86)\end{array}$ & $\begin{array}{c}0.19 \\
(0.10)\end{array}$ \\
\hline Majority Female & $\begin{array}{l}2.1^{* *} \\
(0.80)\end{array}$ & $\begin{array}{l}0.13^{* *} \\
(0.05)\end{array}$ & $\begin{array}{l}3.4^{* * *} \\
(0.86)\end{array}$ & $\begin{array}{c}0.38^{* * *} \\
(0.09)\end{array}$ \\
\hline All Female & $\begin{array}{c}3.3^{*} \\
(1.5)\end{array}$ & $\begin{array}{c}0.20^{*} \\
(0.09)\end{array}$ & $\begin{array}{c}8.6^{* * *} \\
(1.7)\end{array}$ & $\begin{array}{c}0.79^{* * *} \\
(0.13)\end{array}$ \\
\hline \multicolumn{5}{|l|}{ Fixed-effects } \\
\hline Year $\times$ Subcategory & Yes & Yes & Yes & Yes \\
\hline Year $\times$ Team Size & Yes & Yes & Yes & Yes \\
\hline Number of Matched Publications by Inventors & Yes & Yes & & \\
\hline Number of Matched NIH Articles & & & Yes & Yes \\
\hline Observations & 22,666 & 22,238 & 12,834 & 11,554 \\
\hline
\end{tabular}

Robust standard-errors in parentheses

Signif. Codes: ***: 0.001, **: 0.01, *: 0.05 
Table S-46: The number of female-focused and male-focused patents by whether the inventors are majority women (greater than or equal to $50 \%$ ) or majority men (strictly greater than $50 \%$ )

\begin{tabular}{lccc}
\hline \hline & Female-focused patent & Male-focused patent & Not sex-focused \\
\hline Male-majority team & 46,453 & 49,886 & 295,906 \\
Female-majority team & 8,246 & 7,053 & 43,947 \\
\hline \hline
\end{tabular}

Table S-47: Summary statistics for our patent data

\begin{tabular}{lcccccc}
\hline \hline Variable & Mean & Median & Std.Dev. & Max & Min & N \\
\hline Team Size & 2.88 & 2.00 & 2.13 & 33.00 & 1.00 & 430,060 \\
Year & 1998.10 & 1999.00 & 8.36 & 2010.00 & 1976.00 & 430,060 \\
Female Mesh & 0.13 & 0.00 & 0.33 & 1.00 & 0.00 & 430,060 \\
Male Mesh & 0.13 & 0.00 & 0.34 & 1.00 & 0.00 & 430,060 \\
Minority Female Team & 0.15 & 0.00 & 0.36 & 1.00 & 0.00 & 430,060 \\
Majority Female Team & 0.09 & 0.00 & 0.29 & 1.00 & 0.00 & 430,060 \\
All Female Team & 0.04 & 0.00 & 0.19 & 1.00 & 0.00 & 430,060 \\
\hline \hline
\end{tabular}


Table S-48: Summary statistics for our publication data

\begin{tabular}{lcccccc}
\hline \hline Variable & Mean & Median & Std.Dev. & Max & Min & N \\
\hline Team Size & 6.18 & 5.00 & 5.04 & 719.00 & 1.00 & $2,062,695$ \\
Year & 2010.36 & 2010.00 & 5.05 & 2020.00 & 2002.00 & $2,062,695$ \\
Female Mesh & 0.37 & 0.00 & 0.48 & 1.00 & 0.00 & $2,062,695$ \\
Male Mesh & 0.39 & 0.00 & 0.49 & 1.00 & 0.00 & $2,062,695$ \\
Minority Female Team & 0.39 & 0.00 & 0.49 & 1.00 & 0.00 & $2,062,695$ \\
Majority Female Team & 0.25 & 0.00 & 0.44 & 1.00 & 0.00 & $2,062,695$ \\
All Female Team & 0.06 & 0.00 & 0.23 & 1.00 & 0.00 & $2,062,695$ \\
\hline \hline
\end{tabular}

Table S-49: Summary statistics for our patent data, 2002-2010

\begin{tabular}{lcccccc}
\hline \hline Variable & Mean & Median & Std.Dev. & Max & Min & N \\
\hline Team Size & 3.23 & 3.00 & 2.38 & 33.00 & 1.00 & 168,888 \\
Year & 2005.93 & 2006.00 & 2.61 & 2010.00 & 2002.00 & 168,888 \\
Female Mesh & 0.14 & 0.00 & 0.35 & 1.00 & 0.00 & 168,888 \\
Male Mesh & 0.14 & 0.00 & 0.35 & 1.00 & 0.00 & 168,888 \\
Minority Female Team & 0.19 & 0.00 & 0.39 & 1.00 & 0.00 & 168,888 \\
Majority Female Team & 0.11 & 0.00 & 0.31 & 1.00 & 0.00 & 168,888 \\
All Female Team & 0.04 & 0.00 & 0.20 & 1.00 & 0.00 & 168,888 \\
\hline \hline
\end{tabular}

Table S-50: Summary statistics for our publication data, 2002-2010

\begin{tabular}{lcccccc}
\hline \hline Variable & Mean & Median & Std.Dev. & Max & Min & $\mathrm{N}$ \\
\hline Team Size & 5.55 & 5.00 & 3.94 & 416.00 & 1.00 & $1,050,868$ \\
Year & 2006.06 & 2006.00 & 2.54 & 2010.00 & 2002.00 & $1,050,868$ \\
Female Mesh & 0.33 & 0.00 & 0.47 & 1.00 & 0.00 & $1,050,868$ \\
Male Mesh & 0.36 & 0.00 & 0.48 & 1.00 & 0.00 & $1,050,868$ \\
Minority Female Team & 0.38 & 0.00 & 0.49 & 1.00 & 0.00 & $1,050,868$ \\
Majority Female Team & 0.23 & 0.00 & 0.42 & 1.00 & 0.00 & $1,050,868$ \\
All Female Team & 0.05 & 0.00 & 0.22 & 1.00 & 0.00 & $1,050,868$ \\
\hline \hline
\end{tabular}


Patent info

\section{Open structure breast coil and support arrangement for interventional MRI}

A pair of RF quadrature detection coils are fitted in an open support structure that allows easy diagnostic access to all tissue areas of the human female breast when performing interventional MRI. The open structure dual coil arrangement comprises a flat base and a slightly V-shaped bi-planar upper section supported above the lower bat bas circur op cicla openings for acl at 900 intervals around each breast opening and provide large side-open access areas to the breast tissues via the sides of the support structure. The $V$-shape of the upper portion of the coil support structure increases the gap clearance at both left and right sides of the support structure to further enhance access to the breasts. In a preferr embodiment, two complete MRI quadrature detection coils are supported by the structure and each of the vertical support legs house a pair of conductors forming part of a quadrature detection coil winding. The use of quadrature detection coils increases MRI signal detection efficiency and the open structural support arrangement allows increased accessibility to the breast tissues for efficient interventional MR imaging and easy cleaning after use.This invention relates to open structure $\mathrm{RF}$ receive coils and an associated support structure for magnetic resonance imaging (MRI). More specifically, it relates to a quadrature type receive coils and associated support structures for obtaining MR images of the human breast which allows ample access to the breast tissues for permitting interventional MRI procedures. Magnetic resonance (MR) images acquired during surgery can assist a surgeon to accurately locate tissue malignancies, obtain a biopsy from desired locations and ensure successful tissue removal.

Interventional MRI is the magnetic resonance imaging technique (often involving reatime imaging) that allows a surgeon to perform MRI-guided tissue biopsy or surgery. One application of interventional MRl is to guide a surgeon during a biopsy or surgica operation on one or both of the breasts of female patients Interventional MR procedures typically require that an MR signal detection coil have large openings so that a surgeon can have access to the surgical site through the coil with biopsy needle
MeSH Terms

FEMALE
0
Rank: 1

\section{HUMANS}

$\square$ Delete

PRE-ASSIGNED MESH TERMS

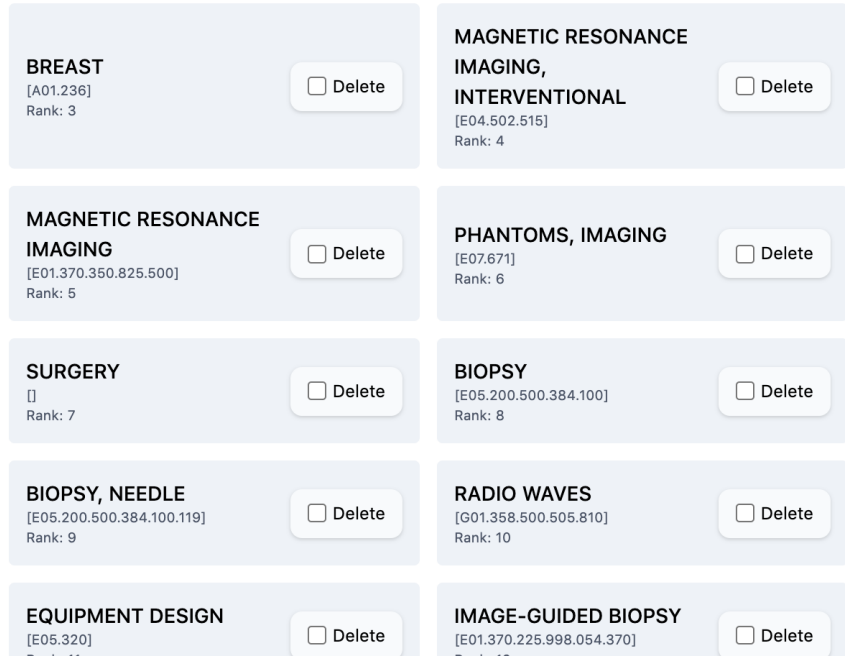

Figure S-1: Screenshot from the web app used by human medical indexers to evaluate our algorithm. The human, female, and male check tags were always displayed at the top, irrespective of whether the MTI classified the patent as matching these tags. 


\section{Patent info}

\section{Targets for breast cancer diagnosis and treatment}

CDNA sequences derived from four novel genes associated with breast cancer are provided. In over about $60 \%$ of the cancer cell lines tested, RNA hybridizing with the CDNAs were substantially more abundant than in normal cells. Most of the cell lines also showed a duplication of the corresponding gene, which probably contributed to the increased level of RNA in the cell. However, for each of the four genes, there were some cell lines which had RNA overabundance without gene duplication. This suggests that the gene product is sufficiently important to the cancer process that cells will use a poly a olypeptides, and antibodies provided by his inven lion are expected to be panticularly useful in diagnosis and treatment of breast cancer. Also provided is a general method for obtaining CDNA with similar properties that may be associated with breast cancer and other malignancies. The present invention relates generally to the field of human genetics. Morespecifically, it relates to the identification of novel genes associated with overabundance of RNA in human cancer, particularly breast cancer. It pertains especially to those genes and the products thereof which may be important in diagnosis and treatment. One of the most pressing health issues today is breast cancer. In the industrial world, about one woman in every nine can expect to develop breast cancer in her lifetime. In the United States, it is the most common cancer amongst women, with an annual incidence of about 175,000 new cases and nearly 50,000 deaths. Despite an ongoing improvement in our understanding of the disease, breas Wathe cancer focused on early dianosis, tolowed by conventonal morms or incervention, particularly surgery and chemotherapy. Such interventions are of limited success, particularly in patients where the tumor has undergone metastasis. There is a pressing need to improve the arsenal of therapies available to provide more precise and more effective treatment in a less invasive way. A promising area for the development of new modalities has emerged from recent understanding of the genetics of cancer. Alteration of gene expression is intimately related to the uncontrolled growth and dedifferentiation that are hallmarks of the disease. There are two classes of

\begin{tabular}{|c|c|c|c|}
\hline $\begin{array}{l}\text { POPULATION GROUPS } \\
\text { [M01.686] } \\
\text { Rank: } 26\end{array}$ & $\square$ Delete & $\begin{array}{l}\text { DIAGNOSIS } \\
\text { [E01] } \\
\text { Rank: } 28\end{array}$ & $\square$ Delete \\
\hline $\begin{array}{l}\text { METHODS } \\
\text { [E05.581] } \\
\text { Rank: 28 }\end{array}$ & $\square$ Delete & $\begin{array}{l}\text { INDUSTRY } \\
\text { [J01.576] } \\
\text { Rank: } 28\end{array}$ & $\square$ Delete \\
\hline $\begin{array}{l}\text { PRESSURE } \\
\text { [G01.374.715] } \\
\text { Rank: } 28\end{array}$ & $\square$ Delete & $\begin{array}{l}\text { SEXUAL BEHAVIOR } \\
\text { [F01.145.802] } \\
\text { Rank: } 28\end{array}$ & $\checkmark$ Delete \\
\hline $\begin{array}{l}\text { ACHIEVEMENT } \\
\text { [F01.658.059] } \\
\text { Rank: } 33\end{array}$ & $\checkmark$ Delete & $\begin{array}{l}\text { TIME } \\
\text { [G01.910] } \\
\text { Rank: } 33\end{array}$ & $\checkmark$ Delete \\
\hline $\begin{array}{l}\text { INTERPERSONAL } \\
\text { RELATIONS } \\
\text { [F-1.829.401] } \\
\text { Rank: } 33\end{array}$ & $\nabla$ Delete & & \\
\hline
\end{tabular}

\section{ADDITIONAL MESH TERMS}

\author{
Search MeSH Terms
}

COMMENTS

Figure S-2: Screenshot from the web app used by human medical indexers to evaluate our algorithm. The top 40 MeSH tags that the MTI assigned to the patent were displayed. The indexers could delete tags they thought did not apply to the patent. 


\section{Patent info}

Sugar transport sequences, yeast strains having improved sugar uptake, and methods of use

Disclosed are nucleic acid constructs comprising coding sequences operably linked to promoter not natively associated with the coding sequence. The coding sequences encode Pichia stipitis proteins that allow recombinant strains of Saccharomyces cerevisiae expressing the protein to grow on xylose, and allow or increase uptake of xylose by Pichia stipitis or Saccharomyces cerevisiae expressing the coding sequences. Expression of the coding sequences enhances uptake of xylose and/or glucose, allowing increased ethanol or xylitol production. Within the United States, ongoing res is directed toward developing alternative energy sources to reduce our dependence on foreign oil and nonrenewable energy. The use of ethanol as a fuel has become increasingly prevalent in recent years. Currently, corn is the primary carbon source used in ethanol production. The use of corn in ethanol production is not economically sustainable and, arguably, may result in increased food costs. In order to meet the increased demand for ethanol, it will be necessary to ferment sugars from other increased demand for ethanol, it will be necessary to ferment sugars from other
biomass, such as agricultural wastes, corn hulls, corncobs, cellulosic materials, pulping wastes, fast-growing hardwood species, and the like. Biomass from most of these sources contains large amounts of xylose, constituting about 20 to $25 \%$ of the total dry weight. Because agricultural residues have a high xylose content, the potential economic and ecologic benefits of converting xylose in these renewable materials to ethanol are significant. In biomass conversion, microorganisms serve as biocatalysts to convert cellulosic materials into usable end products such as ethanol. Efficient biomass conversion in large-scale industrial applications requires a microorganism that can to rete high sugar and ent toler sugars simult

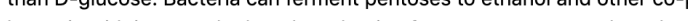
bacteria with improved ethanol production from pentose sugars have been genetically engineered. However, these bacteria are sensitive to low $\mathrm{pH}$ and high concentrations of ethanol, their use in fermentations is associated with co-product formation, and the level of ethanol produced remains too low to make the use of these bacteria in largescale ethanol production

\begin{tabular}{|c|c|c|c|}
\hline Kank: 37 & & Rank: 32 & \\
\hline $\begin{array}{l}\text { ECOLOGY } \\
\text { [H01.277.249] } \\
\text { Rank: } 32\end{array}$ & $\square$ Delete & $\begin{array}{l}\text { BODY WEIGHT } \\
\text { [C23.888.144] } \\
\text { Rank: } 34\end{array}$ & $\square$ Delete \\
\hline $\begin{array}{l}\text { PROMOTER REGIONS, } \\
\text { GENETIC } \\
{\left[\begin{array}{c}6002111.570 .080 .689 .675] \\
\text { Rank: } 35\end{array}\right.}\end{array}$ & $\square$ Delete & $\begin{array}{l}\text { HYDROLASES } \\
\text { [D08.811.277] } \\
\text { Rank: } 35\end{array}$ & $\square$ Delete \\
\hline $\begin{array}{l}\text { BIOLOGICAL FACTORS } \\
\text { [D23] } \\
\text { Rank: } 37\end{array}$ & $\square$ Delete & $\begin{array}{l}\text { DISCLOSURE } \\
\text { [F01.829.401.046] } \\
\text { Rank: } 38\end{array}$ & $\square$ Delete \\
\hline $\begin{array}{l}\text { DISSENT AND DISPUTES } \\
\text { [F02.463.785.373.476] } \\
\text { Rank: } 38\end{array}$ & $\square$ Delete & $\begin{array}{l}\text { COSTS AND COST } \\
\text { ANALYSIS } \\
\text { [N03.219.151] } \\
\text { Rank: } 38\end{array}$ & $\square$ Delete \\
\hline
\end{tabular}

ADDITIONAL MESH TERMS

sugar|

AMINO SUGARS

CARBASUGARS

COUPLING SUGAR

DEOXY SUGARS

DIETARY SUGARS

GUANOSINE DIPHOSPHATE SUGARS

IMINO SUGARS

INVERT SUGAR

NEOSUGAR

NUCLEOSIDE DIPHOSPHATE SUGARS

Figure S-3: Screenshot from the web app used by human medical indexers to evaluate our algorithm. At the bottom of the screen the indexers could add MeSH terms that they thought the MTI had missed. The text box would auto-complete the terms they typed, to ensure they were adding a valid MeSH tag. 

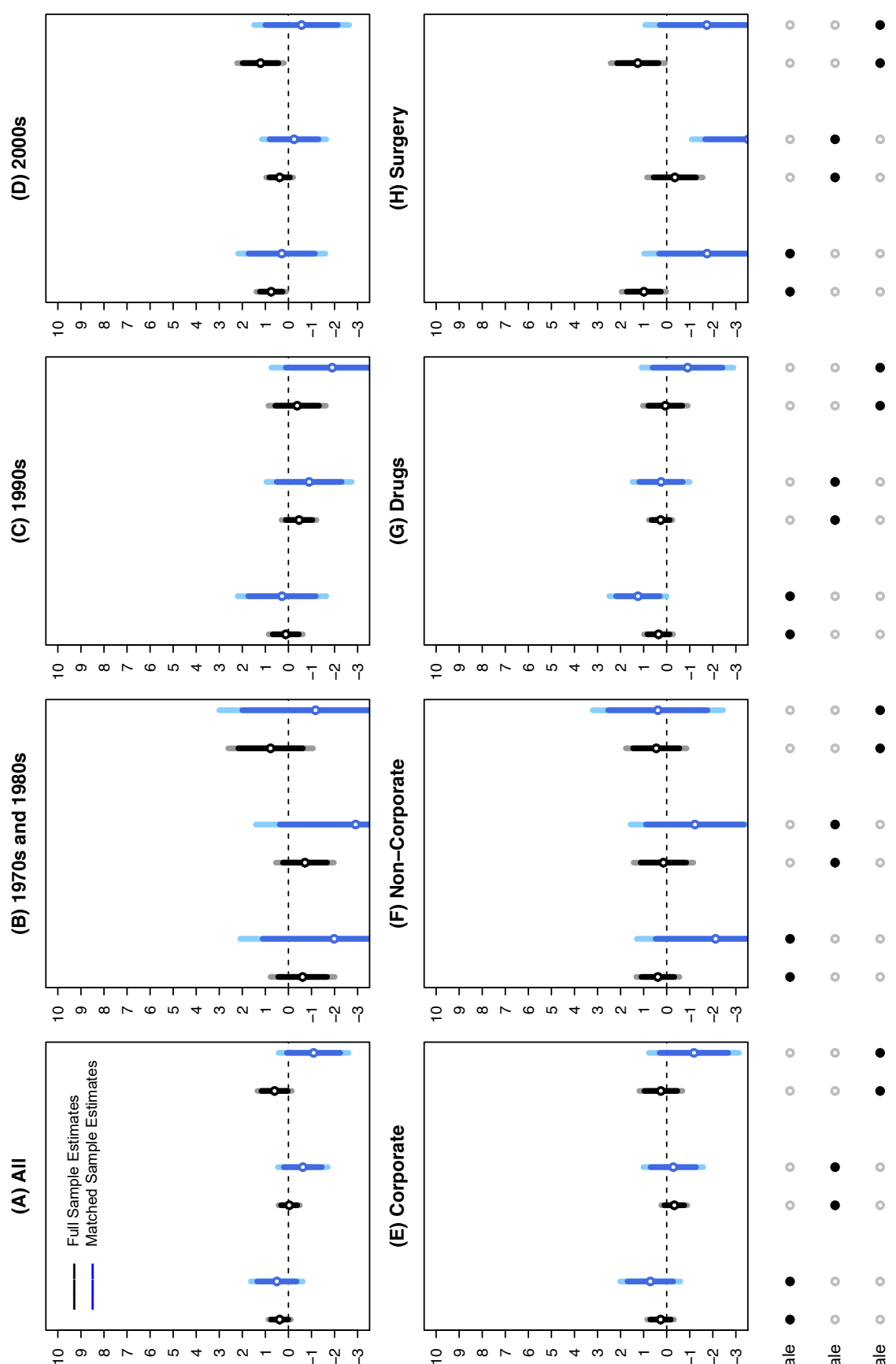

Һоәнヨ рәюеш!ฺง

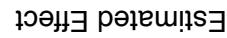

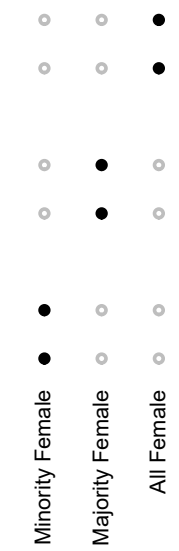

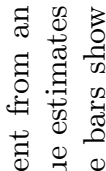

范哭

正

$\stackrel{\circ}{\circ}$

ర

范

0 .

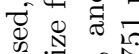

ठ

ఏ

$\times \underset{0}{ } \times$

.

뭉 80

$\pm \pi$

ح

गु 80 के

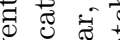

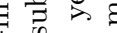

웅

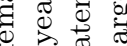

$\approx \frac{1}{2} 0$

过

के

㶾

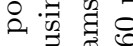

80 .

艺志吕

घ

$\stackrel{0}{0}, \overrightarrow{0}$

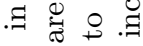

(i) 0

สี

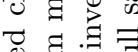

ह.$\exists$

త్ర

.

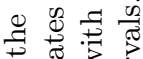

3.्व य

$\overrightarrow{1}_{0}$ है

की प्र

훙 훙 훙

. द्व

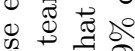

च

E $\frac{0}{0}$

$\ddot{\forall}$ एँ

ต.$\exists$ घ

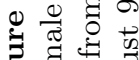

品芒 

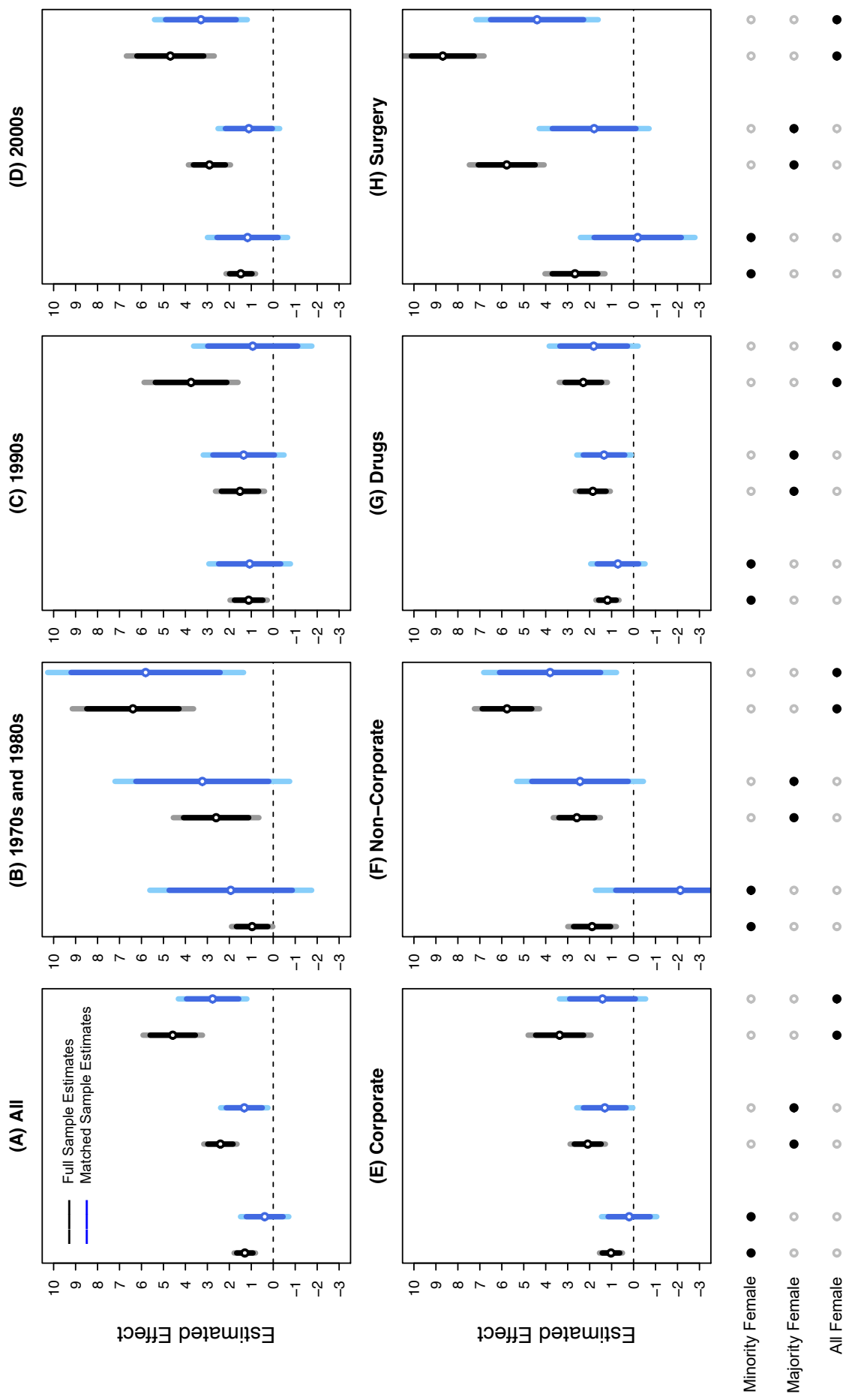

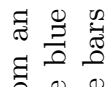

品焉

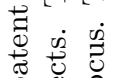

, क्षे

$\circ$ 路

象:

ฮี.

过

需

$\times$

:

๖

สె

过

. 180

त क

范

요 $\times$

ฮี

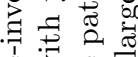

요용

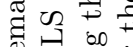

$\circlearrowleft 0$.

ro ?

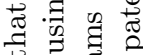

कि 08

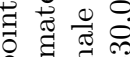

ฉ.ق घ्घ

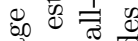

질

㻤.

平

$\therefore \frac{20}{0}$

ن : 0

강 घ द्व

$\tau$.

os 0

过

ज 1 है

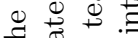

굼

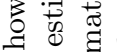

a

ซิ

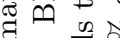

हैं $\frac{0}{0}$ के

ه

ङี च

F. 울

is

ผ่.

월

$\exists$ డ

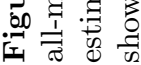



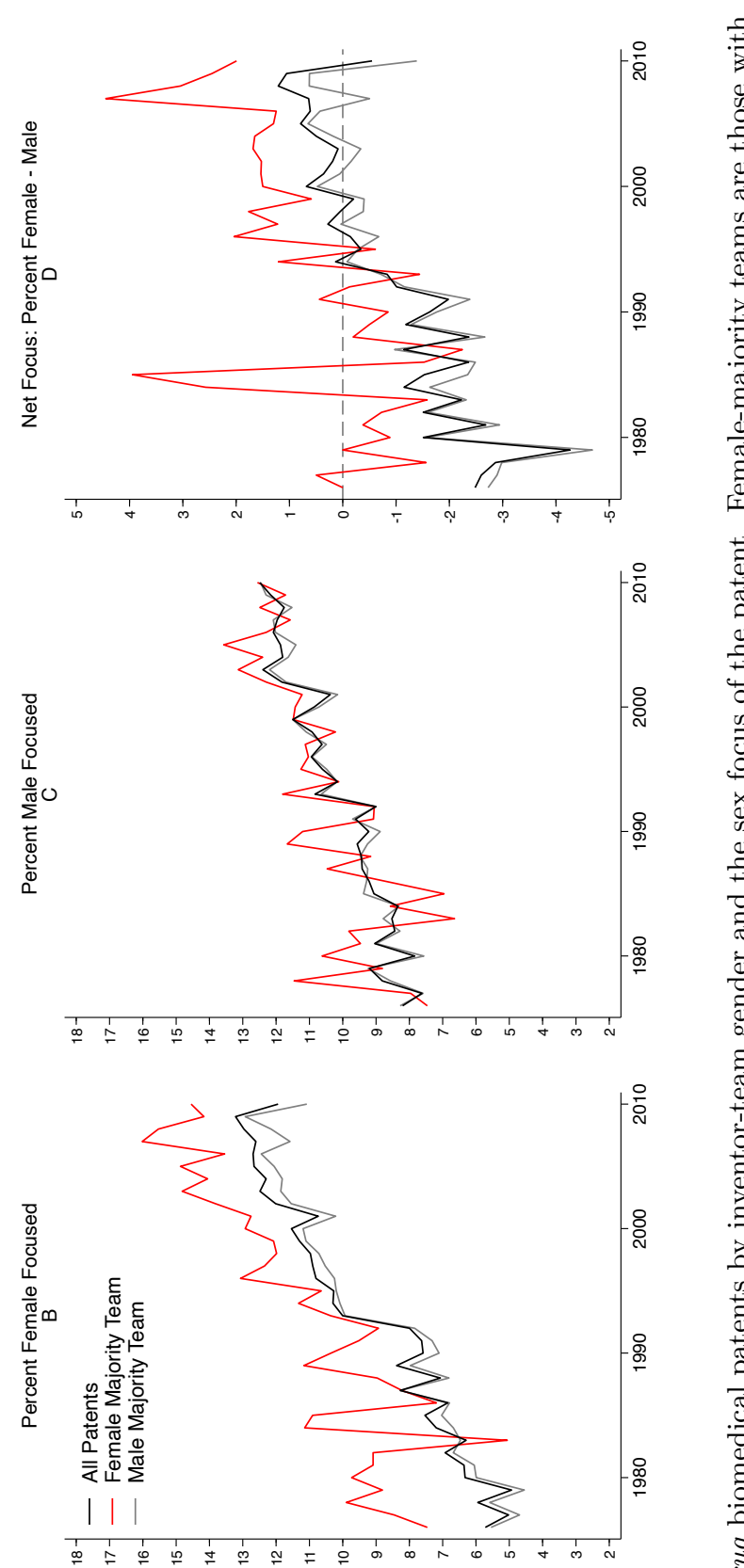

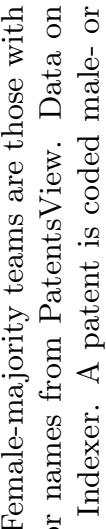

站造

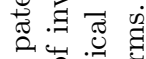

过

证

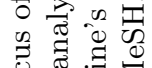

记

希芯苍

政沙

秀帘

䒕芯芯

푼

ชี

$\infty$ 용

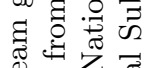

क

훙 छ

항 도

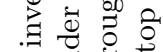

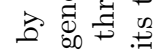

若范苍

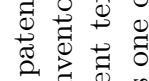

สี.

응 :

胥.

๑ั

옹 苟 छ

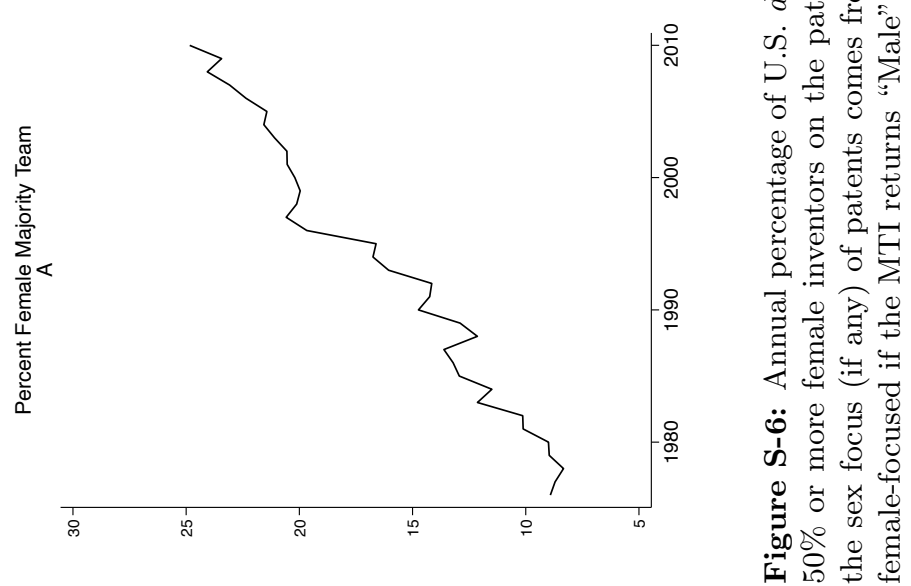



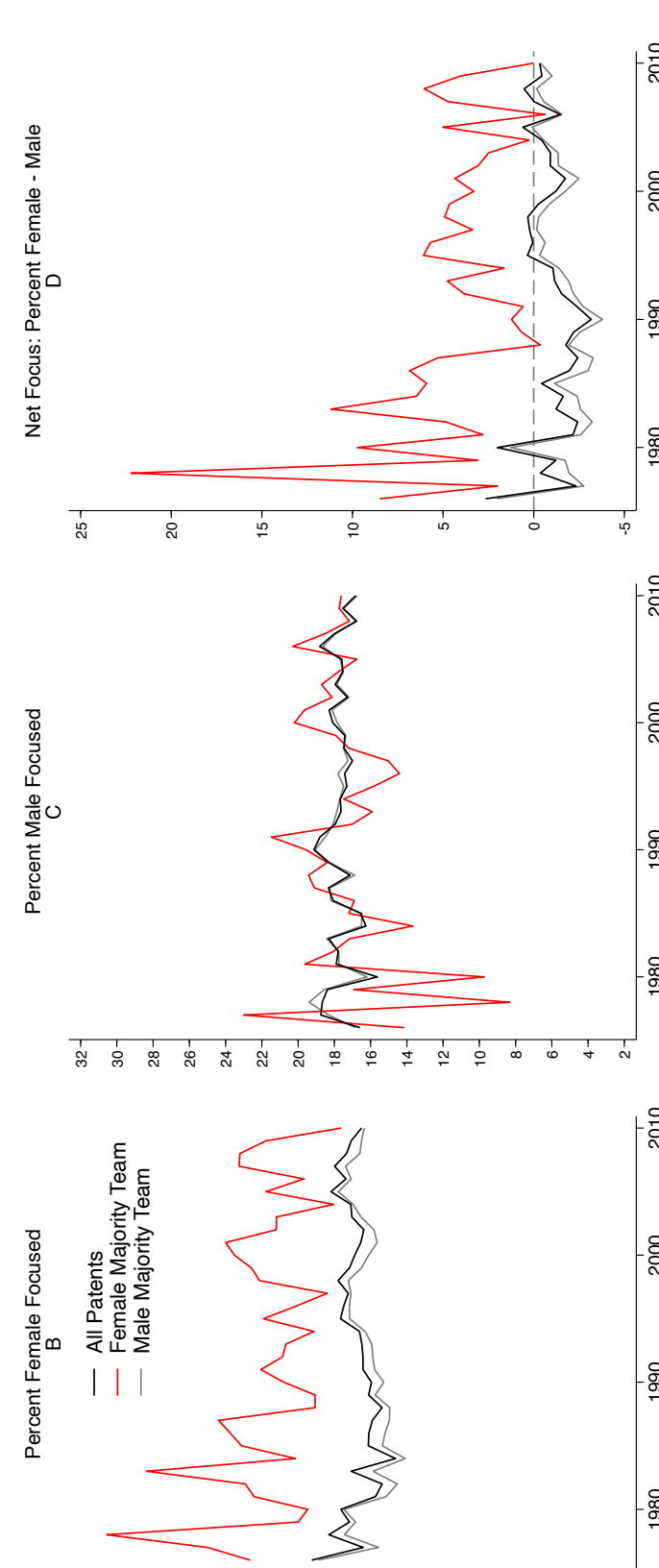

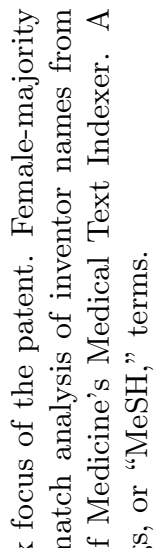

究

䨌

국

สี

ชี

政总

สี

궁 궁

응 ₹

过范

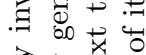

s.

青苛 节

.

范

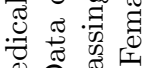

范

훙

苟

苛范

है छ्वे

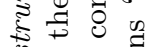

. సे

हี

के

造

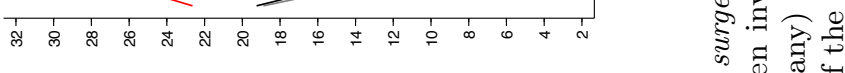

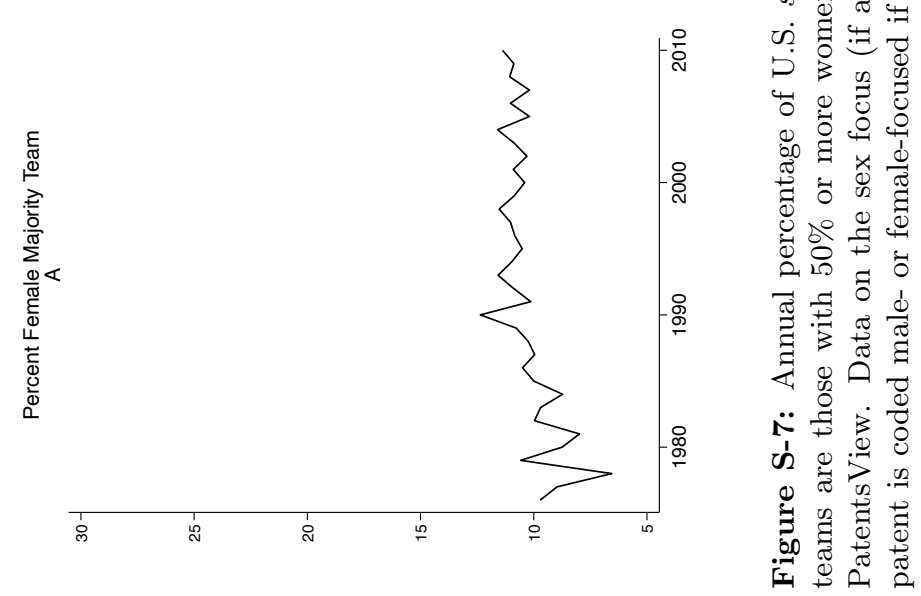




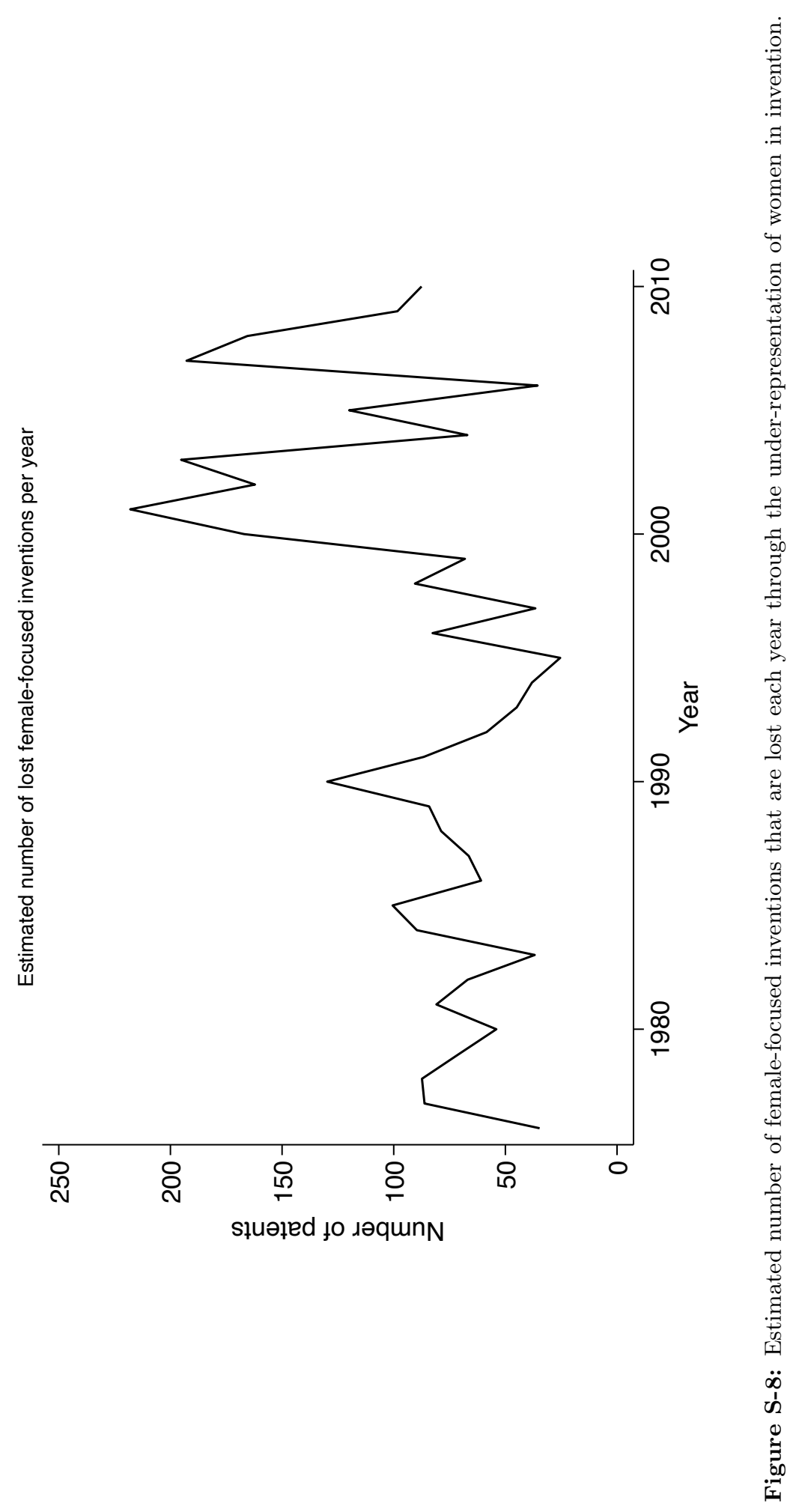

